\title{
Twisting the mirror TBA
}

\author{
Gleb Arutyunov, ${ }^{1}$ Marius de Leeuw and Stijn J. van Tongeren \\ Institute for Theoretical Physics and Spinoza Institute, \\ Utrecht University, 3508 TD Utrecht, The Netherlands \\ E-mail: G.E.Arutyunov@uu.nl, M.deLeeuw@uu.nl, S.J.vanTongeren@uu.nl
}

AbSTRACT: We study finite-size corrections to the magnon dispersion relation in three models which differ from string theory on $\mathrm{AdS}_{5} \times \mathrm{S}^{5}$ in their boundary conditions. Asymptotically, this is accomplished by twisting the transfer matrix in a way which manifestly preserves integrability. In model I all world-sheet fields are periodic, whereas model II represents a particular orbifold of $\mathrm{AdS}_{5} \times \mathrm{S}^{5}$ and model III is a $\beta$-deformed theory. For models I and II we construct the one-particle TBA equations and use them to determine the leading finite-size correction to the asymptotic Bethe equation. We also make some interesting observations concerning the quantization conditions for the momentum. For the same models we compute the leading and for model II the next-to-leading order finite-size corrections to the asymptotic magnon dispersion relation. Furthermore, we apply Lüscher's formulae to compute the leading finite-size corrections in $\beta$-deformed theory. In addition to reproducing known results, we provide new predictions for two-particle states from the $\mathfrak{s l}(2)$ sector, to be confronted with explicit field-theoretic calculations in the dual gauge theory. Finally, we prove that the leading finite-size correction to the energy of an $\mathfrak{s l}(2)$ magnon in orbifold theory is the same as the one for an $\mathfrak{s u}(2)$ magnon in $\beta$-deformed theory for special values of $\beta$. We also speculate that for these values of $\beta$ our result for the nextto-leading order correction in the orbifold model might coincide with the corresponding correction to the energy of an $\mathfrak{s u}(2)$ magnon in $\beta$-deformed theory.

Keywords: AdS-CFT Correspondence, Integrable Field Theories

ArXiv EPrint: 1009.4118

\footnotetext{
${ }^{1}$ Correspondent fellow at Steklov Mathematical Institute, Moscow.
} 


\section{Contents}

$\begin{array}{lll}1 & \text { Introduction } & 2\end{array}$

2 Models: periodic, orbifold and $\beta$-deformed 5

3 Energy and momentum $\quad 12$

4 TBA equations for models I and II $\quad 14$

4.1 Analytic properties of the asymptotic solution 14

$\begin{array}{ll}\text { 4.2 The simplified TBA equations } & 17\end{array}$

$\begin{array}{lll}4.3 & \text { Exact Bethe equations } & 23\end{array}$

5 Finite-size corrections to the dispersion: model I 24

$\begin{array}{lll}5.1 & \text { LO wrapping correction to the energy and momentum } 25\end{array}$

5.2 Quantization of momentum and exact Bethe equation 26

6 Finite-size corrections to the dispersion: model II 28

6.1 LO wrapping correction to the energy and momentum 28

6.2 Quantization of momentum and exact Bethe equation 30

6.3 Comparing energies of $\mathfrak{s l}(2)$ and $\mathfrak{s u}(2)$ states 32

6.4 NLO wrapping correction to the energy 33

$7 \quad$ Finite-size corrections in $\boldsymbol{\beta}$-deformed and physical orbifold theories $\quad \mathbf{3 6}$

$\begin{array}{lll}7.1 & \text { One-particle } \beta \text {-deformed states } & 36\end{array}$

$\begin{array}{lll}7.2 & \text { Two-particle } \beta \text {-deformed states } & 38\end{array}$

$\begin{array}{lll}7.3 & \text { Physical two-particle } \mathfrak{s l}(2) \text { orbifold states } & 39\end{array}$

7.4 Comparing $\beta$-deformed and off-shell orbifold magnons 41

A Twisted transfer matrix 42

B Asymptotic critical points and TBA 44

$\begin{array}{ll}\text { C Wrapping corrections } & 45\end{array}$

$\begin{array}{lll}\text { C.1 Model I } & 46\end{array}$

$\begin{array}{lll}\text { C.2 } & \text { Model II } & 47\end{array}$

$\begin{array}{ll}\text { D Details on the NLO computation } & 47\end{array}$

D.1 The Harmonic number integral 48

D.2 Some integrals 51 


\section{Introduction}

In this paper we employ the mirror TBA in combination with adaptations of Lüscher's perturbative approach to study single magnon configurations for various interesting models, which we discuss in detail below. This has been made possible by various important recent developments in the problem of finding the exact scaling dimensions of $\mathcal{N}=4 \mathrm{SYM}$ gauge-invariant composite operators with finite quantum numbers through the gauge-string correspondence [1].

First of all, Lüscher's perturbative approach [2] has been adapted to the case of the nonLorentz invariant $\mathrm{AdS}_{5} \times \mathrm{S}^{5}$ string sigma-model [3, 4], which led to a determination of the four- and five-loop anomalous dimensions of the Konishi operator $[4,5]$ and, subsequently, of all twist two operators [6-8], see also [9]; the corresponding results agree with first-hand field-theoretic computations [10-12].

Secondly, the Thermodynamic Bethe Ansatz (TBA) equations [13-17] for the $\mathrm{AdS}_{5} \times \mathrm{S}^{5}$ mirror model $^{1}[19]$ have been constructed, based on the corresponding string hypothesis [20]. These equations should provide the tools needed to compute the energy of string states and, therefore, the scaling dimensions of the corresponding operators in the dual gauge theory, as has been nicely demonstrated on the example of the Konishi operator both at weak [21, 22] and intermediate [23, 24] values of the coupling constant. The computation of the five-loop anomalous dimension through the mirror TBA approach has been further extended [25] to all twist two operators and the results obtained agree with the ones based on the generalized Lüscher's formulae [8]. Finally, we note that in general the TBA equations imply a set of functional equations known as the Y-system [26]. The Y-system for the case at hand was conjectured in [27], and its general solution was obtained in [28]. This Y-system has rather unusual analytic properties which are under investigation $[13,17,29,30]$.

In the present paper we continue to explore the mirror TBA approach. We concentrate on the TBA describing a one-particle excited state; a single magnon in the $\mathfrak{s l}(2)$ sector with non-vanishing momentum $p$. This excitation does not exist in the spectrum of closed strings on $\mathrm{AdS}_{5} \times \mathrm{S}^{5}$. The embedding fields describing closed strings must satisfy periodic boundary conditions which results in the level-matching condition; the total momentum of a state must be equal to zero. Of course, the concept of magnons remains of primary importance as it is used to define their scattering matrix; a fundamental building block of the whole integrability approach [31].

The spectral problem for a single magnon can be set up in different ways. One way is to start from the string sigma-model and require the embedding fields to be periodic. In this model, which we call model I, we can study more excitations upon relaxing the level-matching condition. Another possibility is to consider strings in other integrable backgrounds, where the single magnon is a physical excitation. Examples of this type are provided by strings on orbifolds of $\operatorname{AdS}_{5} \times \mathrm{S}^{5}$ and $\beta$-deformed theories.

\footnotetext{
${ }^{1}$ The TBA approach in the AdS/CFT spectral problem was advocated in [18] where it was used to explain wrapping effects in gauge theory.
} 
In general our interest in the single magnon spectral problem is three-fold. Firstly, we would like to understand the general relationship between energy and momentum (dispersion) for integrable models in a finite volume and its dependence on the boundary conditions. Secondly, for theories where a single magnon is a physical excitation, it is interesting to compute finite-size corrections to the magnon energy, providing new data to be compared to the corresponding dual gauge theories. Thirdly, it is important to understand how to extend the TBA approach to a larger class of physical theories.

In this paper we will analyze a one-particle excitation in three different models. The first is the already mentioned model I. Model II is defined by twisting the boundary conditions for sphere bosons by the total momentum of a state. As we will show, this model can be thought of as a particular orbifold of $\mathrm{AdS}_{5} \times \mathrm{S}^{5}$. Finally, model III corresponds to $\beta$-deformed theory. All models share the same world-sheet scattering matrix, which is also the one for strings on $\mathrm{AdS}_{5} \times \mathrm{S}^{5}$ [32-38]. As a consequence, the modified boundary conditions for models II and III in the large $J$ limit $^{2}$ or at weak coupling can be implemented by means of the general theory of integrable twists [39]. Below we will discuss the modified boundary conditions starting from the underlying world-sheet theory.

For models I and II we construct the corresponding TBA equations. Following the same strategy as [17], we assume that the TBA equations for excited states only differ from the ground state ones by the integration contours in the convolution terms. By requiring these equations to be compatible with the large $J$ asymptotic solution given in terms of generalized Lüscher formulae we then fix the shape of the integration contours. Simultaneously, we also determine how the TBA length parameter $L_{T B A}$ is related to $J$. Interestingly enough, for model I there is a noticeable difference between the level-matched and unmatched cases; $L_{T B A}=J+2$ in the first case and $L_{T B A}=J$ in the second. For model II we find an intermediate result for the length; $L_{T B A}=J+1$.

Furthermore, by analytically continuing the TBA equation for the $Y_{1}$-function to the kinematical region of string theory along the lines of [40], we derive an explicit form of the exact Bethe equation $Y_{1}\left(u_{*}\right)+1=0$ for the rapidity variable $u_{*}$. This equation should be viewed as a quantization condition for the particle momentum (rapidity) which replaces the asymptotic Bethe equation for the case of finite $J$.

The large $J$ asymptotic solution is constructed through Lüscher formulae in terms of the transfer matrices in which the boundary conditions of interest are implemented through the corresponding twists. The Lüscher formulae allow us to compute the leading order finite-size corrections to the energy $E_{\mathrm{LO}}$ and momentum $P_{\mathrm{LO}}$. As was found for relativistic integrable models starting from an underlying lattice formulation, see example [41, 42], the energy $E$ and momentum $P$ are given by equations (3.1) and (3.6), respectively. In the case of non periodic boundary conditions, i.e. for models II and III, based on experience with the dual gauge theories, it is plausible to assume that the expression for the asymptotic energy is not modified. The situation regarding $P$ is less clear however, and we just adopt formula (3.6) as a working assumption. We then show that for model I the leading finite-

\footnotetext{
${ }^{2}$ Throughout the paper $J$ stands for the angular momentum of string rotating around the equator of the five-sphere.
} 
size correction to the asymptotic Bethe equation coincides with $P_{\mathrm{LO}}$, while for model II this is not the case. A possible reconciliation of the two is that the introduction of a twist modifies the definition of the momentum in such a way that it becomes compatible with the exact Bethe equation.

For model II, by employing some of the techniques of [5], we have computed the nextto-leading order correction to the energy $E_{\mathrm{NLO}}$ of an $\mathfrak{s l}(2)$ magnon for $J=2,3,4$ and $6 .{ }^{3}$ The results are given in terms of $\zeta$-functions and products thereof; all polygammafunctions arising in various steps of the computation are canceled in the final expressions. In these energy corrections, the term of maximal transcendentality has degree $2 J+3$. We also present results for the energy correction to some two-particle states from the $\mathfrak{s l}(2)$ sector for a generic $\mathbb{Z}_{S}$-orbifold. As opposed to the single magnon, these states satisfy the level-matching condition, i.e. they are physical states of the orbifold model.

Concerning $\beta$-deformed theory, the construction of the TBA equations remains an open problem. Moreover, there is no consensus on the question of integrability of the finite-size model. ${ }^{4}$ Here we restrict ourselves to the study of the leading Lüscher corrections to the energies of states in the $\mathfrak{s u}(2)$ and $\mathfrak{s l}(2)$ sectors. The Lüscher formulae assume factorization of the full world-sheet S-matrix into a product of two $\mathfrak{s u}(2 \mid 2)$-invariant S-matrices, and in this aspect they still reflect the properties of the large $J$ solution. Understanding double wrapping should provide crucial insight on the issue of integrability of the $\beta$-deformed theory in finite volume.

Recently, wrapping energy corrections were found in $\beta$-deformed SYM [44, 45], which sparked attempts to reproduce these from finite size effects in the dual string theory. Close to what we consider here, an approach of obtaining the leading finite-size corrections to the energies of $\mathfrak{s u}(2)$ states in $\beta$-deformed theories via twisting has been implemented in [46]. However, the twist we are using is slightly different from the one in [46] and we justify it from the world-sheet point of view. At the same time, our transfer matrix is also different from the one in [46], because it contains extra momentum-dependent prefactors needed to satisfy the generalized unitarity condition [19]. As a net result, the $Y_{Q}$-functions in the $\mathfrak{s u}(2)$ sector agree with the ones found in [46] for any number of excitations, and therefore in particular the Lüscher corrections obtained there for a single $\mathfrak{s u}(2)$ magnon coincide with ours. Additionally, in [47] the $\beta$-deformed analog of the Konishi state was studied by means of twisting certain elements of the S-matrix. Our twist incorporates this result, but derives it from a different starting point.

We then turn our attention to the $\mathfrak{s l}(2)$ sector in $\beta$-deformed theory with $\beta \neq \frac{n}{J}$, where $n$ is an integer. We determine the leading order corrections to the energy of two-particle states with $J=2,3,5$ and give explicit formulae that can be readily used to compute

\footnotetext{
${ }^{3}$ Here the results take a nicely presentable form.

${ }^{4}$ For instance, the equations which encode a class of finite-gap solutions of the $\beta$-deformed string sigma model have been discussed in [43]. However, these equations have been derived under an assumption that certain non-linear constraints are satisfied (in the limit $\beta \rightarrow 0$ these constraints turn into the global conserved charges that do not belong to the Cartan subalgebra of $\mathrm{SO}(6)$ ). It is unclear for the moment how to extend the corresponding approach to encompass all finite-gap solutions. We thank Sergey Frolov for the discussion of this point.
} 
energy corrections for any $J$ desired, providing new predictions to be confronted with future calculations in the dual gauge theory. We point out that for rational values $\beta=\frac{n}{J}$ the correction starts at an order of $g^{2}$ higher than for generic $\beta$.

We also make a detailed comparison of orbifold and $\beta$-deformed theories and show that for $\beta=\frac{n}{J}$ the leading order finite-size correction to the energy of an $\mathfrak{s l}(2)$ magnon in the orbifold model is the same as the corresponding quantity for an $\mathfrak{s u}(2)$ magnon in $\beta$-deformed theory, in full agreement with the conjecture by [48]. We point out that if this interesting relation between the two theories continues to hold beyond the leading order, our result for $E_{\mathrm{NLO}}$ found for the orbifold model will simultaneously give the NLO correction to the energy of an $\mathfrak{s u}(2)$ magnon in $\beta$-deformed theory for these special values of $\beta$.

The paper is organized as follows. In the next section we introduce the three models and discuss implementation of the corresponding twists at the level of transfer matrices. Section 3 contains some generalities on energy and momentum in the TBA approach. Section 4 is devoted to the construction of the TBA equations for models I and II. In sections 5 and 6 we compute the leading finite-size corrections to the energy and momentum in models I and II, respectively. Section 6 also contains some results on the next-to-leading order correction to the energy of the $\mathfrak{s l}(2)$ magnon in model II. Section 7 contains our results for $\beta$-deformed theories. Some definitions and technical details are relegated to appendices A, $\mathrm{C}$ and D. In appendix B we briefly discuss the issue of critical points for the asymptotic solution of the TBA equations.

We note here that sections 2 and 3 are of general interest, which combined with section 6 contain the background and explicit results on the leading order energy corrections in the orbifold theory, while combined with section 7 they contain the background and explicit results for $\beta$-deformed theory and two particle $\mathfrak{s l}(2)$ orbifold states. Sections 4,5 and 6 contain the results which are important for next-to-leading order (and higher) corrections and our observations regarding momentum quantization.

\section{Models: periodic, orbifold and $\beta$-deformed}

An important peculiarity of the string sigma-model on $\mathrm{AdS}_{5} \times \mathrm{S}^{5}$ is that the fermions exhibit unusual periodicity conditions related to the total momentum $p[49,50]$. Indeed, in the light-cone gauge one of the fields, an angle $\phi$ which parametrizes the five-sphere, is unphysical and must be solved in terms of physical (transversal) fields. The equation of motion for $\phi$ then implies

$$
\phi(2 \pi)-\phi(0)=p
$$

On the other hand, in order to make the world-sheet fermions neutral under the isometry $\phi \rightarrow \phi+$ constant, and simultaneously bring the Wess-Zumino term of the string action to a local form, we need to perform a field redefinition of the fermions $\psi \rightarrow e^{\frac{i}{2} \phi} \psi$. As a result, the new fermions satisfy the twisted boundary conditions

$$
\psi(2 \pi)=e^{\frac{i}{2} p} \psi(0) .
$$


Since we aim to describe closed strings, all the sphere bosons must be periodic which leads to the level-matching condition $p=2 \pi m$, where $m$ is the winding number. As a result, the theory splits into sectors with periodic and anti-periodic boundary conditions for fermions, depending on whether $m$ is even or odd.

Moving on, we recall that in the large $J$ limit the symmetry algebra of the string sigmamodel coincides with two copies of the centrally extended $\mathfrak{s u}(2 \mid 2)$ superalgebra with the same central charge depending on $p[34,51]$. The fact that the 16 physical fields ( 8 bosons and 8 fermions) transform under two copies of $\mathfrak{s u}(2 \mid 2)$ suggests to treat this representation as a tensor product $4 \times 4$, where 4 is a fundamental four-dimensional representation of $\mathfrak{s u}(2 \mid 2)$ with two bosons and two fermions.

In order to treat a single magnon, we have to relax the level-matching condition. It is then easy to see that it is impossible to twist the boundary conditions for factorized worldsheet fields in such a way as to have all sphere and AdS bosons strictly periodic, while fermions satisfying the twisted boundary conditions (2.2). In the following we therefore introduce three models which have the same field content as the string sigma-model on $\mathrm{AdS}_{5} \times \mathrm{S}^{5}$ but differ in their boundary conditions. Within these models we will be able to study the spectral problem for a single magnon.

Model I - Periodic fields. In this model one assumes that all fields - bosons and fermions - are periodic. The level-matching condition is not imposed allowing to consider a single magnon with non-vanishing momentum. Clearly, the periodic boundary conditions preserve all the global symmetries of the original light-cone string sigma-model.

Model II - Orbifold. In this model one twists the boundary conditions for bosonic fields $y_{a}$ and $y_{\dot{a}}$, where $a=1,2$ and $\dot{a}=\dot{1}, \dot{2}$ :

$$
\left(\begin{array}{l}
y_{1}(2 \pi) \\
y_{2}(2 \pi)
\end{array}\right)=\left(\begin{array}{cc}
e^{i \alpha_{\ell}} & 0 \\
0 & e^{-i \alpha_{\ell}}
\end{array}\right)\left(\begin{array}{l}
y_{1}(0) \\
y_{2}(0)
\end{array}\right), \quad\left(\begin{array}{l}
y_{\dot{j}}(2 \pi) \\
y_{\dot{2}}(2 \pi)
\end{array}\right)=\left(\begin{array}{cc}
e^{i \alpha_{r}} & 0 \\
0 & e^{-i \alpha_{r}}
\end{array}\right)\left(\begin{array}{l}
y_{\dot{1}}(0) \\
y_{\dot{2}}(0)
\end{array}\right),
$$

while fermions $\theta_{\alpha}$ and $\theta_{\dot{\alpha}}$ kept untwisted and so the AdS fields as well. Here we should distinguish two possibilities for choosing the left and right twists:

$$
\alpha_{\ell}=\alpha_{r} \quad \text { or } \quad \alpha_{\ell}=-\alpha_{r} .
$$

The twists above will lead to a modification of the boundary conditions for two of the four fields $Y_{a \dot{a}}$. Together with the two fields contained in the complex $Z$ field which is necessarily unaffected by the twist, these fields parametrize the five-sphere. Furthermore the physical fermions $\theta_{a \dot{\alpha}}=y_{a} \theta_{\dot{\alpha}}$ and $\eta_{\dot{a} \alpha}=\theta_{\alpha} y_{\dot{a}}$ inherit the twisted periodicity conditions

$$
\begin{array}{rlrl}
\theta_{1 \dot{\alpha}}(2 \pi) & =e^{i \alpha_{\ell}} \theta_{1 \dot{\alpha}}(0), & & \eta_{\dot{1} \alpha}(2 \pi)=e^{i \alpha_{r}} \eta_{\dot{1} \alpha}(0), \\
\theta_{2 \dot{\alpha}}(2 \pi)=e^{-i \alpha_{\ell}} \theta_{2 \dot{\alpha}}(0), & & \eta_{\dot{2} \alpha}(2 \pi)=e^{-i \alpha_{r}} \eta_{\dot{2} \alpha}(0) .
\end{array}
$$

The two twists in equation (2.3) lead to essentially equivalent theories, so in what follows we have chosen to explicitly treat the case $\alpha_{\ell}=-\alpha_{r}$.

A particularly interesting situation arises when a twist is chosen to be

$$
\alpha_{\ell}=\frac{2 \pi n}{S}
$$


where $n$ and $S$ are integers. In this case the twisted boundary conditions coincide with the ones for string theory on the orbifold $\operatorname{AdS}_{5} \times S^{5} / \Gamma$, where $\Gamma$ is a cyclic subgroup $\mathbb{Z}_{S}$ in $\mathfrak{s u}(2) \subset \mathfrak{s u}(4)$, see $[52,53]$. The canonical element generating $\mathbb{Z}_{S}$ is $\omega=e^{\frac{2 \pi i}{S}}$ and various values of $n, n=0, \ldots, S-1$ describe the corresponding twisted sectors. Since two of the three complex scalars, $Z$ and $Y_{11}$, do not undergo twisting, the orbifold model preserves an $\mathfrak{s u}(2)$ part of the original $\mathfrak{s u}(4)$ R-symmetry and, therefore, has $\mathcal{N}=2$ supersymmetry.

Yet another possibility, which we will be mostly interested in, is to chose $\alpha_{\ell}=-\alpha_{r}=\frac{P}{2}$, where $P$ is the total momentum of a state. Excitations in this model with these boundary conditions are embedded in the orbifold model. Indeed, at large $J$ the momentum of a single magnon is subjected to the quantization condition $p=\frac{2 \pi n}{J}, n \in \mathbb{Z}$, which is nothing else but the Bethe equation, cf. our discussion at the end of this section. Therefore, for fixed $J$ we deal with a magnon in the twisted sector corresponding to $\omega^{n}=e^{\frac{i \pi n}{J}}$ of the orbifold theory. As we will see, the momentum $p$ receives finite-size corrections starting at order $g^{2 J+4}$; these effects are compatible with the quantization condition, and hence preserve the orbifold structure. For LO and NLO corrections to the energy of a single magnon the difference between $p$ and the total momentum will not play any role.

In both model I and II we will study states from the $\mathfrak{s l}(2)$ sector. For states from this sector the level-matching condition implies that the total momentum of a state must be equal to zero. In particular therefore a single $\mathfrak{s l}(2)$ magnon is not a physical state of the orbifold theory. In what follows we will therefore study one-magnon configurations by relaxing the level-matching condition. In section 7 we also compute the leading finite-size corrections for a few physical two-particle states of a generic orbifold.

It is worth pointing out that the orbifold boundary conditions with the twist parameter chosen to be proportional to the total momentum introduced here, are similar but not equivalent to those of $\beta$-deformed theories. Nevertheless, as will show this model exhibits an interesting relation to $\beta$-deformed theories.

Model III - $\boldsymbol{\beta}$-deformations. This is a model which describes an exactly marginal deformation of $\mathrm{AdS}_{5} \times \mathrm{S}^{5}$ superstring theory. It has been introduced in [54] and further studied in [43, 55-58]. The model admits a natural generalization to a more general non-supersymmetric background, which is obtained as a three-parameter deformation of $\mathrm{AdS}_{5} \times \mathrm{S}^{5}$. The latter can be regarded as the same $\mathrm{AdS}_{5} \times \mathrm{S}^{5}$ string sigma-model but with twisted boundary conditions for three isometric angles $\phi_{i}$ of $S^{5}$, namely,

$$
\phi_{i}(2 \pi)-\phi_{i}(0)=-2 \pi \epsilon_{i j k} \gamma_{j} J_{k}, \quad i=1,2,3 .
$$

In these $\gamma$-deformed theories, the $\gamma_{i}$ are three deformation parameters and the $J_{i}$ are conserved Noether charges corresponding to the shift isometries in the $\phi_{i}$ direction. Because of the presence of the $J_{i}$, the deformations depend on the state of interest. For the symmetric case

$$
\gamma_{1}=\gamma_{2}=\gamma_{3}=\beta
$$

with $\beta$ real, the deformation preserves 8 supercharges and it is dual to the $\mathcal{N}=1$ superconformal field theory which is an exactly marginal deformation of $\mathcal{N}=4$ super Yang-Mills theory [54]. 
To continue, let us recall that the transverse bosonic fields describing the five-sphere can be combined in a matrix $Y$

$$
Y=\left(\begin{array}{cc}
Y_{1 \dot{1}} & Y_{1 \dot{2}} \\
Y_{2 \dot{1}} & Y_{2 \dot{2}}
\end{array}\right)=\left(\begin{array}{cc}
a e^{-i \phi_{2}} & b e^{-i \phi_{3}} \\
b e^{i \phi_{3}} & -a e^{i \phi_{2}}
\end{array}\right)
$$

where we exhibit explicitly the dependence on two isometric angles $\phi_{2}$ and $\phi_{3}$ [50]. The other angle $\phi_{1}$ parametrizes the complex field

$$
Z=\frac{1-a^{2}-b^{2}}{1+a^{2}+b^{2}} e^{i \phi_{1}}
$$

Thus, the three $\phi$ 's together with $a$ and $b$ provide a parametrization of the five-sphere. We see that equation (2.6) implies the following twisted boundary conditions for sphere bosons

$$
Y(2 \pi)=\left(\begin{array}{cc}
e^{i \pi \beta\left(J_{2}-J_{3}\right)} & 0 \\
0 & e^{-i \pi \beta\left(J_{2}-J_{3}\right)}
\end{array}\right) Y(0)\left(\begin{array}{cc}
e^{i \pi \beta\left(2 J_{1}-J_{2}-J_{3}\right)} & 0 \\
0 & e^{-i \pi \beta\left(2 J_{1}-J_{2}-J_{3}\right)}
\end{array}\right) .
$$

Obviously, these conditions are inherited from the following periodicity conditions for $y_{a}$ (left bosons) and $y_{\dot{a}}$ (right bosons)

$$
\begin{aligned}
\left(\begin{array}{l}
y_{1}(2 \pi) \\
y_{2}(2 \pi)
\end{array}\right) & =\left(\begin{array}{cc}
e^{i \pi \beta\left(J_{2}-J_{3}\right)} & 0 \\
0 & e^{-i \pi \beta\left(J_{2}-J_{3}\right)}
\end{array}\right)\left(\begin{array}{l}
y_{1}(0) \\
y_{2}(0)
\end{array}\right) \\
\left(\begin{array}{l}
y_{\dot{1}}(2 \pi) \\
y_{\dot{2}}(2 \pi)
\end{array}\right) & =\left(\begin{array}{cc}
e^{i \pi \beta\left(2 J_{1}-J_{2}-J_{3}\right)} & 0 \\
0 & e^{-i \pi \beta\left(2 J_{1}-J_{2}-J_{3}\right)}
\end{array}\right)\left(\begin{array}{l}
y_{\dot{1}}(0) \\
y_{\dot{2}}(0)
\end{array}\right) .
\end{aligned}
$$

Also, we have

$$
Z(2 \pi)=Z(0) e^{2 \pi \beta\left(J_{2}-J_{3}\right)} .
$$

As was mentioned above, in the light-cone gauge the field $\phi \equiv \phi_{1}$ is non-dynamical and satisfies equation (2.1). Thus, $p=2 \pi \beta\left(J_{2}-J_{3}\right)$ is the level-matching condition in $\beta$ deformed theory. Finally, we mention that the fermions $\theta_{\alpha}$ and $\theta_{\dot{\alpha}}$ have standard periodic boundary conditions, while the physical fermions $\theta_{a \dot{\alpha}}=y_{a} \theta_{\dot{\alpha}}$ and $\eta_{\alpha \dot{a}}=\theta_{\alpha} y_{\dot{a}}$ will inherit the twisted boundary conditions from the bosons.

In $\beta$-deformed theory we will be interested in two kind of states which we call states from the $\mathfrak{s u}(2)$ and $\mathfrak{s l}(2)$ sectors respectively. The states from $\mathfrak{s u}(2)$ have $J_{3}=0$ and are characterized by two charges $J \equiv J_{1}$ and $M \equiv J_{2}$; the level-matching condition is $p=2 \pi \beta M$. For $J$ large such a state can be represented as

$$
\underbrace{A_{1 \mathrm{i}}^{+}\left(p_{1}\right) \ldots A_{1 \mathrm{i}}^{+}\left(p_{M}\right)}_{M}|0\rangle,
$$

where $A_{1 \mathrm{i}}^{+}$is a (bosonic) creation operator from the corresponding Faddeev-Zamolodchikov algebra [59]. The gauge theory operators dual to the states from $\mathfrak{s u}(2)$ have schematically the form $\operatorname{tr}\left(\Phi^{M} Z^{J}\right)$, where a complex scalar $\Phi$ is dual to $Y_{11}$. A single $\mathfrak{s u}(2)$ magnon with $p=2 \pi \beta$ is a physical excitation of $\beta$-deformed theory. 
The states from $\mathfrak{s l}(2)$ have a single charge $J$ and a spin $S$, and in the large $J$ limit can be represented as

$$
\underbrace{A_{3 \dot{3}}^{+}\left(p_{1}\right) \ldots A_{3 \dot{3}}^{+}\left(p_{S}\right)}_{S}|0\rangle
$$

where 3 and $\dot{3}$ are fermionic indices. Since $J_{2}=M=0$, the level matching condition ${ }^{5}$ is $p=0$. A single $\mathfrak{s l}(2)$ magnon can be considered off-shell, but in this case it is not a physical excitation of $\beta$-deformed theory. The dual gauge theory operators are schematically $\operatorname{tr}\left(D^{S} Z^{J}\right)$.

Twisted transfer matrix. In the large $J$ limit the spectrum of the models discussed above is captured by the asymptotic Bethe Ansatz equations (the Bethe-Yang equations) which are constructed taking into account the corresponding boundary conditions. In the framework of the Algebraic Bethe Ansatz, (modified) boundary conditions are accounted for by means of twisting the transfer matrix. Now consider $M$ string theory particles characterized by the $u_{*}$-plane rapidities $u_{1}, \ldots, u_{M}$ or, equivalently, with momenta $p_{1}, \ldots, p_{M}$. Consider also an auxiliary particle with rapidity $v$ corresponding to a bound state representation $\pi_{Q}$ of $\mathfrak{s u}(2 \mid 2)$ with the bound state number $Q$. Scattering this auxiliary particle through $N$ particles gives rise to a monodromy matrix

$$
\mathbb{T}(v \mid \vec{u})=\prod_{i=1}^{M} \mathbb{S}_{a i}\left(v, u_{i}\right) .
$$

Here $\mathbb{S}_{a i}\left(v, u_{i}\right)$ is the S-matrix which describes scattering of the auxiliary particle with another one with rapidity $u_{i}$. As a matrix acting on the auxiliary space, $\mathbb{T}(v \mid \vec{u})$ satisfies the fundamental commutation relations

$$
\mathbb{S}_{12}\left(v_{1}, v_{2}\right) \mathbb{T}_{1}\left(v_{1} \mid \vec{u}\right) \mathbb{T}_{2}\left(v_{2} \mid \vec{u}\right)=\mathbb{T}_{2}\left(v_{2} \mid \vec{u}\right) \mathbb{T}_{1}\left(v_{1} \mid \vec{u}\right) \mathbb{S}_{12}\left(v_{1}, v_{2}\right) .
$$

We can also introduce a twisted transfer matrix

$$
T(v \mid \vec{u})=\operatorname{Tr}\left[\pi_{Q}(g) \mathbb{T}(v \mid \vec{u})\right],
$$

where an element $g$ is a twist and trace is taken over the auxiliary space. If $g$ is such that $\left[\mathbb{S}_{12}, g \otimes g\right]=0$, then the fundamental commutation relations imply that $T(v \mid \vec{u})$ commute for different values of $v$ and therefore define a set of commuting charges. For the string S-matrix we are interested in $g \in \mathrm{SU}(2) \times \mathrm{SU}(2)$ and as such the most general twist will involve six arbitrary parameters. Here the first $\mathrm{SU}(2)$ corresponds to the AdS space, while the second one corresponds to the five-sphere.

Postponing development of the general theory to the future, ${ }^{6}$ we note that for all cases of present interest the twist is of the form $g=1 \otimes K$, where

$$
K=\left(\begin{array}{cc}
e^{i \alpha} & 0 \\
0 & e^{-i \alpha}
\end{array}\right) .
$$

\footnotetext{
${ }^{5}$ For simplicity we restrict ourselves to the case of no winding.

${ }^{6}$ A generic twist corresponds to describing the most general orbifold and $\gamma$-deformed theories; it breaks global symmetries of the model and brings the Bethe roots at infinity to finite positions in the complex plane $[57,60]$.
} 


\begin{tabular}{|l|l|l|l|l|}
\hline Sector & \multicolumn{2}{|c|}{$\mathfrak{s l}(2)$} & \multicolumn{2}{c|}{$\mathfrak{s u}(2)$} \\
\hline Factor & L & $\mathrm{R}$ & $\mathrm{L}$ & $\mathrm{R}$ \\
\hline Periodic (I) & 0 & 0 & 0 & 0 \\
\hline Orbifold (II) & $p / 2$ & $\pm p / 2$ & $p / 2$ & $\pm p / 2$ \\
\hline$\beta$-deformed (III) & 0 & $2 \pi J \beta$ & $\pi M \beta$ & $(2 J-M) \pi \beta$ \\
\hline
\end{tabular}

Table 1. The twists for the different models in the $\mathfrak{s l}(2)$ and $\mathfrak{s u}(2)$ sectors. The off-shell $\mathfrak{s u}(2)$ magnon with zero twist has not been investigated.

- For model I $\alpha=0$, i.e. twist is absent for both left and right copies of $\mathfrak{s u}(2 \mid 2)$.

- For model II we have two options

$$
\alpha_{\ell}=\alpha_{r}=\frac{p}{2} \quad \text { or } \quad \alpha_{\ell}=-\alpha_{r}=\frac{p}{2},
$$

for the left and $\alpha_{\ell}$ and $\alpha_{r}$ refer to the left and the right transfer matrices, respectively.

- For $\beta$-deformed theory the left and right transfer matrices corresponding to left and right copies of $\mathfrak{s u}(2 \mid 2)$ are twisted with

$$
\alpha_{\ell}=\pi \beta\left(J_{2}-J_{3}\right), \quad \alpha_{r}=\pi \beta\left(2 J_{1}-J_{2}-J_{3}\right)
$$

for the left and the right transfer matrices, respectively.

For the reader's convenience we have summarized the twists for the different models in the $\mathfrak{s l}(2)$ and $\mathfrak{s u}(2)$ sectors in table 1.

The $Q$-particle bound state representations of $\mathfrak{s u}(2 \mid 2)$ have dimension $4 Q$ and are of two types; symmetric and anti-symmetric. Hence we can define two transfer matrices, $T_{s, 1}$ and $T_{a, 1}$ corresponding to symmetric and anti-symmetric bound state representations respectively. The relations between $T_{1, s}$ and $T_{a, 1}$ are schematically depicted in figure 1 . These transfer matrices are now used to construct the asymptotic Y-functions $Y_{Q}$ in the $\mathfrak{s l}(2)$ and $\mathfrak{s u}(2)$ sectors. $^{7}$ The corresponding expressions are given by the generalized Lüscher formula [4];

$$
Y_{Q}^{o}(v)=e^{-J \tilde{\mathcal{E}}_{Q}(v)} T^{\ell}(v \mid \vec{u}) T^{r}(v \mid \vec{u}) \prod_{i=1}^{M} S_{\mathfrak{s l}(2)}^{Q 1_{*}}\left(v, u_{i}\right) .
$$

Here $\tilde{\mathcal{E}}_{Q}$ is the energy of a mirror $Q$-particle, $S_{\mathfrak{s l}(2)}^{Q 1 *}(v, u)$ denotes the S-matrix with the first and second argument in the mirror and string regions, and $T^{\ell, r}$ is the left, respectively right twisted $\mathfrak{s u}(2 \mid 2)$ transfer matrix. In order to avoid any possible confusion we will indicate the grading of the transfer matrices by the subscript $\mathfrak{s l}(2)$ or $\mathfrak{s u}(2)$.

\footnotetext{
${ }^{7}$ The reader should not confuse the asymptotic $Y_{Q^{-}}^{o}$ and exact $Y_{Q^{-}}$functions. Since we are mainly interested here in the asymptotic solution, we will often omit the superscript "o", for instance, in section 4 where the TBA equations are discussed.
} 
Below we discuss the asymptotic Bethe equations which in the present approach arise from the condition $Y_{1 *}\left(u_{j}\right)=-1$, the latter being the equations for the string rapidities $u_{j}$. Here $Y_{1 *}$ is the asymptotic $Y_{1}(v)$ function analytically continued to the kinematic region of string theory.

Bethe equations in the $\mathfrak{s l}(2)$ sector. In appendix A we present the full eigenvalues of the twisted transfer matrix $T_{Q, 1}^{\mathfrak{s l}(2)}$, as well as the corresponding auxiliary Bethe equations which can be easily obtained by generalizing the result of [61] found for the untwisted case. For states from the $\mathfrak{s l}(2)$ sector we have $T^{\ell}=T^{r}=T_{Q, 1}^{\mathfrak{s l}(2)}$, where the eigenvalue $T_{Q, 1}^{\mathfrak{s l}(2)}$ now does not involve auxiliary Bethe roots and is given by

$$
\begin{aligned}
& T_{Q, 1}^{\mathfrak{s l}(2)}(v \mid \vec{u})=1+\prod_{i=1}^{M} \frac{\left(x^{-}-x_{i}^{-}\right)\left(1-x^{-} x_{i}^{+}\right)}{\left(x^{+}-x_{i}^{-}\right)\left(1-x^{+} x_{i}^{+}\right)} \frac{x^{+}}{x^{-}} \\
& -2 \cos \alpha \sum_{k=0}^{Q-1} \prod_{i=1}^{M} \frac{x^{+}-x_{i}^{+}}{x^{+}-x_{i}^{-}} \sqrt{\frac{x_{i}^{-}}{x_{i}^{+}}}\left[1-\frac{\frac{2 i k}{g}}{v-u_{i}+\frac{i}{g}(Q-1)}\right]+\sum_{m= \pm} \sum_{k=1}^{Q-1} \prod_{i=1}^{M} \lambda_{m}\left(v, u_{i}, k\right) .
\end{aligned}
$$

Definitions of various quantities entering the last formula can be found in appendix A; $\cos \alpha$ is a twist of the bosonic eigenvalues. The subscript of the transfer matrix indicates the anti-symmetric bound state representation which an auxiliary particle transforms in upon continuation of its spectral parameter from the mirror to string theory kinematical region. The transfer matrix is normalized as $T_{1 * 1}^{\mathfrak{s l}(2)}=1$, so that the asymptotic Bethe equations in the $\mathfrak{s l}(2)$ sector have the form

$$
1=e^{i p_{j} J} \prod_{i \neq j}^{M} S_{\mathfrak{s l}(2)}\left(u_{j}, u_{i}\right) .
$$

Here $S_{\mathfrak{s l}(2)}^{1_{*} 1_{*}}\left(u_{j}, u_{i}\right) \equiv S_{\mathfrak{s l}(2)}\left(u_{j}, u_{i}\right)$ is the usual $\mathfrak{s l}(2)$ sector S-matrix. In particular, for a single excitation this gives

$$
1=e^{i p J}
$$

From here, note that in none of the models we consider the twist enters the $\mathfrak{s l}(2)$ Bethe equations. This of course should be the case because in all our models, including the $\beta$-deformed one, the $\mathfrak{s l}(2)$ bosons have usual periodic boundary conditions.

Bethe equations in the $\mathfrak{s u}(2)$ sector. In the $\mathfrak{s u}(2)$ sector the basic object needed to build up the $Y_{Q}$ functions through the generalized Lüscher formulae is the transfer matrix $T_{Q, 1}^{\mathfrak{s u}(2)}$, the left and right copies of which are generically twisted differently. For the twisted $T_{Q, 1}^{\mathfrak{s u}(2)}$ one has

$$
\begin{aligned}
T_{Q, 1}^{\mathfrak{s u}(2)}= & (Q+1) \prod_{i=1}^{M} \frac{x^{-}-x_{i}^{-}}{x^{+}-x_{i}^{-}} \sqrt{\frac{x^{+}}{x^{-}}}-Q e^{-i \alpha} \prod_{i=1}^{M} \frac{x^{-}-x_{i}^{+}}{x^{+}-x_{i}^{-}} \sqrt{\frac{x^{+} x_{i}^{-}}{x^{-} x_{i}^{+}}}- \\
& -Q e^{i \alpha} \prod_{i=1}^{M} \frac{x^{-}-x_{i}^{-}}{x^{+}-x_{i}^{-}} \frac{x_{i}^{-}-\frac{1}{x^{+}}}{x_{i}^{+}-\frac{1}{x^{+}}} \sqrt{\frac{x^{+} x_{i}^{+}}{x^{-} x_{i}^{-}}}+(Q-1) \prod_{i=1}^{M} \frac{x^{-}-x_{i}^{+}}{x^{+}-x_{i}^{-}} \frac{x_{i}^{-}-\frac{1}{x^{+}}}{x_{i}^{+}-\frac{1}{x^{+}}} \sqrt{\frac{x^{+}}{x^{-}}}
\end{aligned}
$$




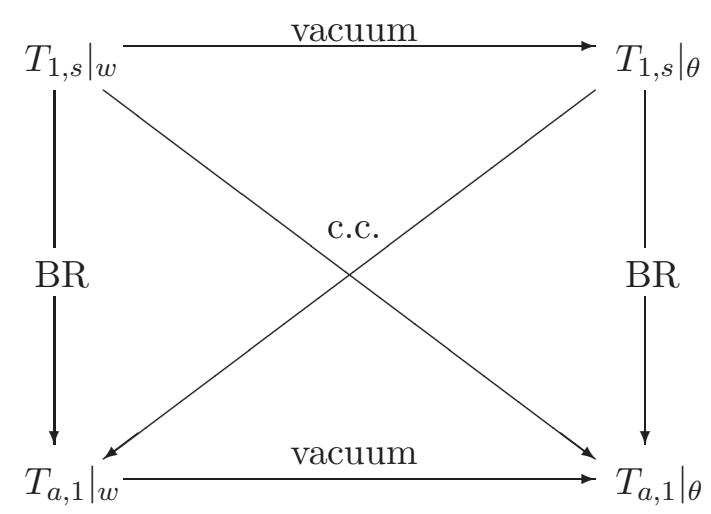

Figure 1. The relations between eigenvalues of the transfer matrix calculated for (anti-)symmetric representations on a bosonic $(w)$ and fermionic $(\theta)$ vacuum [62]. BR stands for the BazhanovReshetikhin formula [63], c.c. for complex conjugation or equivalently for switching the scattering matrix $S$ for $S^{-1}$.

This time the subscript of the transfer matrix indicates the symmetric bound state representation which an auxiliary particle transforms in upon continuation of its spectral parameter from the mirror to string theory kinematical region. The asymptotic Bethe equations now read

$$
-1=Y_{1_{*}}\left(u_{k}\right)=e^{i p_{k} J} e^{-i\left(\alpha^{\ell}+\alpha^{r}\right)} \prod_{i=1}^{M} S_{\mathfrak{s l}(2)}\left(u_{k}, u_{i}\right)\left(\frac{x_{k}^{-}-x_{i}^{+}}{x_{k}^{+}-x_{i}^{-}} \sqrt{\frac{x_{k}^{+} x_{i}^{-}}{x_{k}^{-} x_{i}^{+}}}\right)^{2} .
$$

In particular, for a single excitation we have ${ }^{8}$

$$
1=e^{i\left(p J-\alpha_{\ell}-\alpha_{r}\right)} .
$$

\section{Energy and momentum}

In the TBA approach for the $\mathrm{AdS}_{5} \times \mathrm{S}^{5}$ mirror model, the energy of an $M$-particle state from the $\mathfrak{s l}(2)$-sector is given by

$$
E=J+\sum_{i=1}^{M} \mathcal{E}\left(p_{i}\right)-\frac{1}{2 \pi} \sum_{Q=1}^{\infty} \int \mathrm{d} v \frac{d \tilde{p}^{Q}}{d v} \log \left(1+Y_{Q}(v)\right) .
$$

Here the integration runs over a real rapidity line of the mirror theory and $\tilde{p}^{Q}$ are momenta of the mirror $Q$-particles. Moreover, $\mathcal{E}(p)$ is the asymptotic energy of a string theory particle with momentum $p$, given by

$$
\mathcal{E}(p)=\sqrt{1+4 g^{2} \sin ^{2} \frac{p}{2}} .
$$

\footnotetext{
${ }^{8}$ Recall that $S_{\mathfrak{s l}(2)}(u, u)=-1$.
} 
The last term in the formula (3.1) can be understood as the finite-size correction to the asymptotic, i.e. large $J$, dispersion relation.

As in the general case, for a single excitation the value of $p$, or equivalently the value of the rapidity $u(p)$

$$
u(p)=\frac{1}{g} \cot \frac{p}{2} \sqrt{1+4 g^{2} \sin ^{2} \frac{p}{2}},
$$

is found from the exact Bethe equation $Y_{1 *}(u)=-1$. As was pointed out in section 2, when $J$ becomes large, the exact Bethe equations should turn into the asymptotic Bethe Ansatz equations, which for a single excitation are simply

$$
e^{i p J}=1 \quad \Longrightarrow \quad p J=2 \pi n, \quad n=0,1, \ldots\left[\frac{J}{2}\right]
$$

Analogously, in various known integrable models the TBA approach leads to the following expression for the total momentum

$$
P=\sum_{i=1}^{M} p_{i}-\frac{1}{2 \pi} \sum_{Q=1}^{\infty} \int \mathrm{d} v \frac{d \tilde{\mathcal{E}}^{Q}}{d v} \log \left(1+Y_{Q}(v)\right),
$$

where here $\tilde{\mathcal{E}}^{Q}$ would be the energy of a mirror $Q$-particle; the integral term in the large formula can be viewed as a finite-size correction to the total momentum of string theory particles. For a single magnon one gets

$$
P=p-\frac{1}{2 \pi} \sum_{Q=1}^{\infty} \int \mathrm{d} v \frac{d \tilde{\mathcal{E}}^{Q}}{d v} \log \left(1+Y_{Q}(v)\right),
$$

where $p$ is determined by equations (3.4). It might appear slightly surprising that even for a single particle its momentum is modified by the finite-size correction. One might offer a physical interpretation of $P$ as being the combined momentum of a string particle and the mirror background. The correction term would then be thought of as the back-reaction of the mirror background to the particle; the former also gets into motion when a string theory particle is excited.

On general grounds we could expect that in a finite-size theory it is the momentum $P$ which must be quantized. Thus, we are led to two apparently different quantization conditions

$$
e^{i P J}=1 \quad \Longleftrightarrow \quad Y_{1_{*}}(u)=-1
$$

which nevertheless should be compatible with each other. For model I we will find perturbative evidence that this is indeed the fact. However, in model II we will show that with the same definition of $P$ only one of the two quantization conditions holds, namely the second one. 


\section{TBA equations for models I and II}

In this section we start by discussing the analytic properties of the asymptotic solution and then show how these properties are taken into account in the excited state TBA equations. Finally, we summarize the complete set of excited state TBA equations for a single magnon in both the twisted and untwisted cases. These equations will be subsequently used to find NLO corrections to the energy and momentum through the TBA approach.

\subsection{Analytic properties of the asymptotic solution}

The analytic properties of the asymptotic Y-functions play an essential role in constructing the excited state TBA equations. This is because the integration contours for the excited state TBA equations are not known a priori. Demanding the TBA equations to be compatible with the asymptotic solution provides the necessary information to fix the integration contours. ${ }^{9}$

In general we could expect that with the level-matching condition relaxed, the analytic properties of the asymptotic solution would become more intricate, and as it turns out this is indeed the case for the models of interest here. In addition we will see that the analytic properties of the corresponding solutions in models I and II are considerably different. Now as the TBA equations essentially involve three types of terms

$$
\log Y, \quad \log (1 \pm Y) \quad \text { and } \quad \log \left(1-Y^{-1}\right),
$$

this means that as far as integration along a contour in the complex plane is concerned, we are interested in those points where

$$
Y(r)=0, \infty, \pm 1 .
$$

When changing either the coupling constant $g$ or the momentum $p$ of a state these points $r$ move in the complex plane, and as we will see, these points can either always lie on one side of the integration contour, or cross it dynamically when changing either $g$ or $p$. Because the next sections of the paper will be directly concerned with the small $g$ behaviour of the TBA equations, we relegate a discussion of so-called critical behaviour in $g$ to appendix B, focusing here on the points $r$ that are relevant already at weak coupling.

Before moving on to discussing the particular zeros and poles in either model, we want to briefly comment on how zeroes and poles of the asymptotic Y-functions and points where they equal \pm 1 are related, and how to relate these to zeroes and poles of the transfer matrices; we will illustrate this by using the $Y_{M \mid v w}$-functions as a simple example. Analogous relationships hold for other Y-functions as well.

In both model I and model II, because the denominator remains finite, zeroes of $Y_{M \mid v w}$ are inherited from zeroes of the transfer matrices used to construct them. ${ }^{10}$ The presently

\footnotetext{
${ }^{9}$ Of course, this approach is based on the assumption that the exact solution develops no new singularities in addition to those which are already present in the asymptotic solution.

${ }^{10}$ The asymptotic Y-functions are constructed in terms of transfer matrices based on the underlying symmetry group of the model $[64,65]$; in the context of the string sigma model the corresponding asymptotic Y-functions were presented in [27].
} 


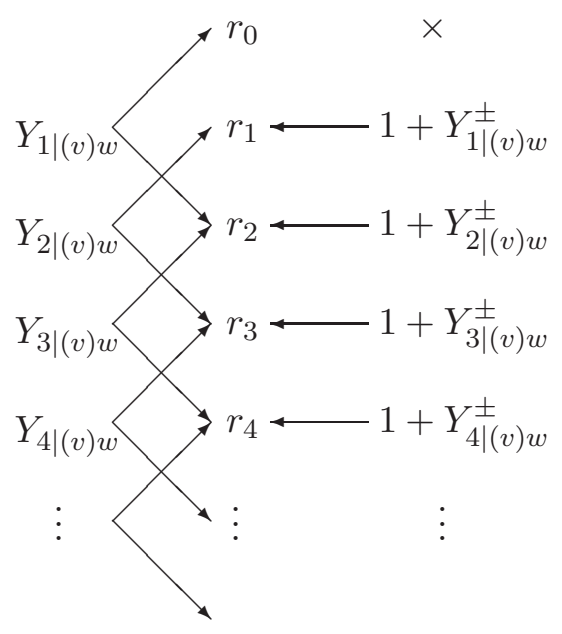

Figure 2. The relationship between zeroes of $Y_{M \mid(v) w}(+1)$. Note that $r_{M}$ here stands for the full set of zeroes of $T_{M+1}$.

relevant $Y$-functions are constructed in terms of the transfer matrices as follows;

$$
\begin{aligned}
Y_{M \mid w} & =\frac{T_{1, M} T_{1, M+2}}{T_{2, M+1}}, \quad Y_{-}=-\frac{T_{2,1}}{T_{1,2}}, \quad Y_{+}=-\frac{T_{2,3} T_{2,1}}{T_{1,2} T_{3,2}}, \\
Y_{M \mid v w} & =\frac{T_{M, 1} T_{M+2,1}}{T_{M+1,2}}=\frac{T_{M, 1} T_{M+2,1}}{T_{M+1,1}^{-} T_{M+1,1}^{+}-T_{M, 1} T_{M+2,1}}
\end{aligned}
$$

Here the \pm superscript denotes the usual shift of the argument by $\pm i / g$, and all transfer matrices are implicitly written in the $\mathfrak{s l}(2)$ grading. From the last expression we immediately see that a zero $r$ of $T_{M, 1}$ is also a zero of $Y_{M \mid v w}$, and it is clear that shifting this zero by $\pm i / g$ we get the value of the rapidity where a lower Y-function becomes equal to minus one; $Y_{M-1 \mid v w}\left(r^{ \pm}\right)=-1$, where $r^{ \pm} \equiv r \pm i / g$.

While the construction of $Y_{M \mid w}$ in terms of transfer matrices does not immediately allow for a simple interpretation like the one above, it should come as no surprise that similarly a zero of $Y_{M \mid w}$ can give rise to points where $Y_{M-1 \mid w}$ is equal to -1 . These relations for $Y_{M \mid w}$ and $Y_{M \mid v w}$ have been summarized in the diagram in figure 2. Here we will not be concerned with poles, as they play no role in either model at small coupling.

With these ideas in mind, we are left with a rather elaborate discussion of all zeroes and poles of the asymptotic Y-functions in both models.

Model I. In the untwisted case, the $Y_{M \mid v w}$-functions exhibit zeroes, while $Y_{+}$and $Y_{-}$ exhibit both zeroes and poles that have to be taken into account already at weak coupling. As mentioned earlier, these zeroes are directly linked to zeroes of the transfer matrices; here each transfer matrix $T_{M}$ has a single zero, which we will denote by $r_{M-1}$. These zeroes have a natural ordering as $r_{M}>r_{M+1}$ and they give rise to two zeroes of $Y_{M \mid v w}, r_{M-1}$ and $r_{M+1}$, and in addition to $Y_{M \mid v w}\left(r_{M}^{ \pm}\right)=-1$. Also $r_{1}$ is a zero of both $Y_{+}$and $Y_{-}$, as 
also the transfer matrices in their denominators remain finite at this point. Finally, at $u^{ \pm}$ the numerators and denominators of $Y_{ \pm}$have poles. However in $Y_{-}$they cancel, whereas in $Y_{+}$the numerator has a pole of higher order, giving rise to poles of $Y_{+}$at $u^{ \pm}$.

Furthermore $Y_{+}=1$ at the points $\mathbf{r}_{0}$ and its conjugate $\overline{\mathbf{r}}_{0}$. These points give rise to a string of poles of $Y_{M \mid w}$ and $Y_{M \mid v w}$, which are located at $\mathbf{r}_{M}$ and $\overline{\mathbf{r}}_{M}$, where $\mathbf{r}_{M}=\mathbf{r}_{0}+i M / g$. These point play no role at weak coupling however; they are discussed in more detail in appendix B.

Model II. As mentioned above, twisting significantly modifies the analytic properties of the asymptotic solution. First of all both $Y_{M \mid w}$ and $Y_{M \mid v w}$ now have zeroes. In the case of $Y_{M \mid v w}$, according to our general discussion, these zeroes originate from the ones of the transfer matrices; this time for $M>1$ each transfer matrix has two zeroes, which are real or purely imaginary, and come with opposite signs. Explicitly, we have

$$
T_{M, 1}\left( \pm r_{M-1}\right)=0
$$

where for $M=1$ we only have $T_{1,1}\left(-r_{0}\right)=0$. In fact, we were able to guess the exact analytic form of these zeroes. It looks very intriguing

$$
r_{M}=\sqrt{u^{2}-M(M+2) \mathcal{E}^{2} / g^{2}}
$$

where $u$ is the Bethe root and $\mathcal{E}$ is nothing but the asymptotic energy (3.1); note that $r_{0}=u$. For $p>0$, the last formula can be rewritten as

$$
r_{M}=\frac{\mathcal{E}(p)}{g \sin \frac{p}{2}} \sqrt{1-(M+1)^{2} \sin ^{2} \frac{p}{2}} .
$$

In general we thus have $Y_{M \mid v w}\left( \pm r_{M \pm 1}\right)=0$. For $M=1$, while the story is a little different, the conclusion remains the same; $Y_{1 \mid v w}$ still has $u$ as a zero although it is not a zero of $T_{1}$, this is due to the pole of $T_{2}$ at $u^{-}$. Of course, these zeroes give rise to points $r_{M}^{ \pm}$where $Y_{M \mid v w}\left( \pm r_{M}^{ \pm}\right)=-1$.

Moving on, all $Y_{M \mid w}$-functions turn out to have two zeroes which are always real. We will denote these zeroes by $\rho_{M-1}$ and $\rho_{M+1}$;

$$
Y_{M \mid w}\left(\rho_{M-1}\right)=Y_{M \mid w}\left(\rho_{M+1}\right)=0 .
$$

Again we were able to guess their form, which fascinatingly enough is given by

$$
\rho_{M}=-u_{M+1}
$$

where

$$
u_{M} \equiv \frac{M}{g} \cot \frac{M p}{2} \sqrt{1+4\left(\frac{g}{M}\right)^{2} \sin ^{2} \frac{M p}{2}},
$$

where one immediately recognizes that $\rho_{0}=-u$. Similar to $Y_{M \mid v w}, 1+Y_{M \mid w}$ has zeroes at the points $\rho_{M} \pm i / g$.

Finally the $Y_{ \pm}$functions each have the same two zeroes, ${ }^{11} Y_{+}\left( \pm r_{1}\right)=Y_{-}\left( \pm r_{1}\right)=0$. The points $\pm r_{M}^{ \pm}$which gave rise to roots of $1+Y_{M \mid v w}$, here for $M=0$ split nicely into 


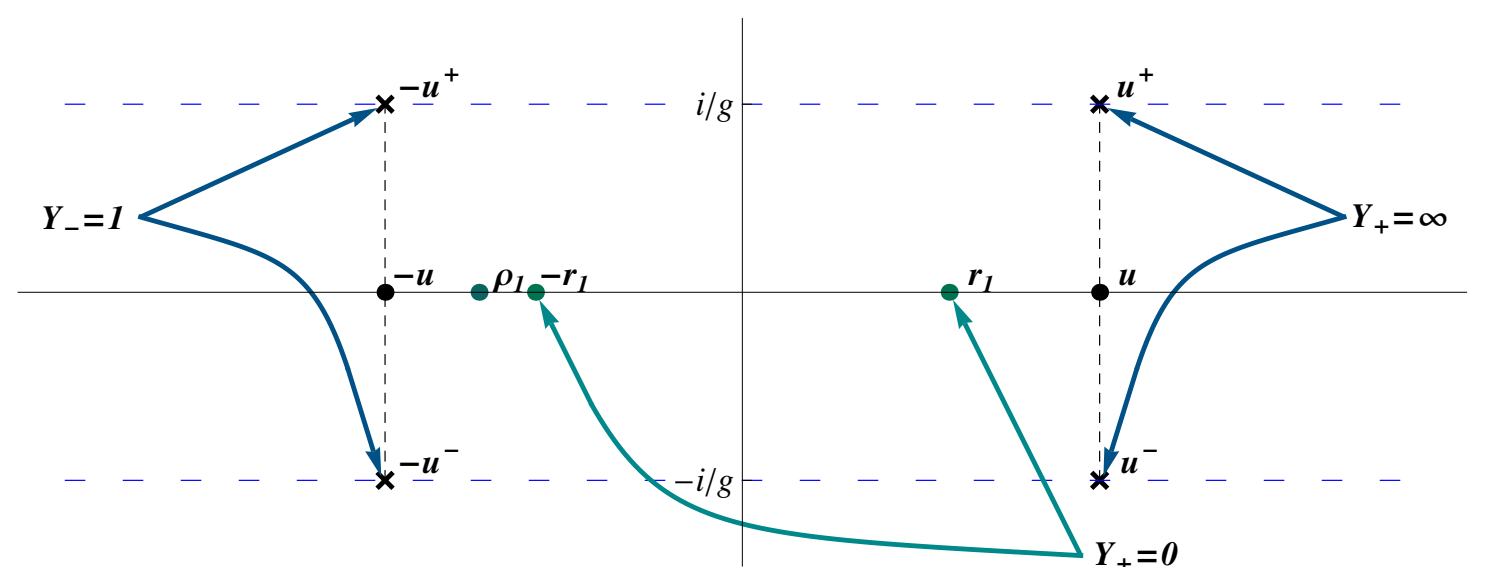

Figure 3. Special points for $Y_{ \pm}$in the complex $v$ plane. Note that $r_{1}$ can also be imaginary as opposed to real as is illustrated here.

\begin{tabular}{|c|c|c|c|c|c|}
\hline Y-function & Zeros I & Poles I & Zeros II & Poles II & Cuts \\
\hline$Y_{+}$ & $r_{1}$ & $u^{ \pm}$ & $\pm r_{1}$ & $u^{ \pm}, \rho_{1}$ & $0, \pm 2$ \\
\hline$Y_{+}-1$ & $\mathbf{r}_{0}, \overline{\mathbf{r}}_{0}$ & & - & & \\
\hline$Y_{-}$ & $r_{1}$ & - & $\pm r_{1}$ & $\rho_{1}$ & $0, \pm 2$ \\
\hline$Y_{-}-1$ & - & & $-u^{ \pm}$ & & \\
\hline$Y_{M \mid w}$ & - & $\mathbf{r}_{M}, \overline{\mathbf{r}}_{M}$ & $\rho_{M \pm 1}$ & - & $\pm M, \pm(M+2)$ \\
\hline$Y_{M \mid w}+1$ & - & & $\rho_{M}^{ \pm}$ & & \\
\hline$Y_{M \mid v w}$ & $r_{M \pm 1}$ & $\mathbf{r}_{M}, \overline{\mathbf{r}}_{M}$ & $\pm r_{M \pm 1}$ & - & $\pm M, \pm(M+2)$ \\
\hline$Y_{M \mid v w}+1$ & $r_{M}^{ \pm}$ & & $\pm r_{M}^{ \pm}$ & & \\
\hline
\end{tabular}

Table 2. Essential analytic structure of the asymptotic solution for a single magnon in both the twisted (right) and untwisted (left) case. A dash indicates absence of a zero or pole, while the empty spots would have information that is already in the table. The branch cuts are $(-\infty, 2] \cup[2, \infty)$, shifted up and down in the complex plane by the multiples of $i / g$ indicated. N.B. $-u^{ \pm}$should be read as $-u \pm \frac{i}{g}$ everywhere.

zeroes of $1-Y_{-}$at $-u^{ \pm}$and poles of $Y_{+}$at $u^{ \pm}$giving a very symmetric situation, as illustrated in figure 3 . In addition both $Y_{+}$and $Y_{-}$have a pole at $\rho_{1}$.

For the reader's convenience, an overview of the relevant roots and poles in both models is presented in table 2 .

\subsection{The simplified TBA equations}

In order to obtain the excited state TBA equations, following [17], we employ the contour deformation trick. In short we assume that the ground state and excited state TBA equations differ only by the choice of the integration contours, and upon deforming the integration contours of the excited state TBA equations to the ground state ones, we pick up contributions of extra singularities, leading to the appearance of new driving terms in the

\footnotetext{
${ }^{11}$ Not surprising since $Y_{+}$and $Y_{-}$share a common factor, which then of course is the source of the zeroes.
} 

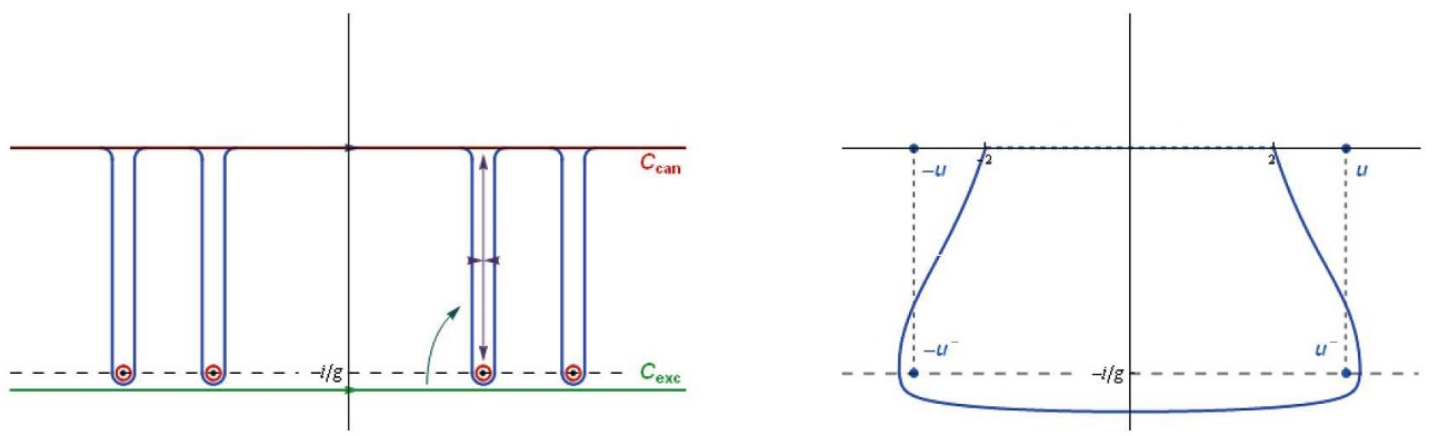

Figure 4. The integration contour for all Y-functions except $Y_{ \pm}$(left) and the integration contour for $Y_{ \pm}$(right).

excited state TBA equations. This procedure is very similar in spirit to the Dorey-Tateo trick [40]. Below we discuss the choice of the integration contours in detail.

The integration contours for all functions $Y_{Q}, Y_{M \mid w}$ and $Y_{M \mid v w}$ can be taken the same, hence we will speak of the integration contour for $Y_{Q}, Y_{M \mid w}$ and $Y_{M \mid v w}$ functions. This contour needs to be chosen such that it encompasses the pole of $\log \left(1+Y_{Q}\right)$ at the Bethe roots in the string region, which is naturally motivated by the fact that this gives a driving term for each Bethe root, see [17] for an explicit discussion. With this requirement it is only natural that the integration contour should run a little bit below the line $-i / g$ after deforming it, and in fact we find that accepting or discarding contributions of zeroes and poles according to this choice of the contour, we obtain a set of TBA equations which is perfectly satisfied by the asymptotic solution. Returning the integration contour to the real mirror line, we pick up a contribution from all zeroes and poles that lie between the real line and the line $-i / g$, as well as the usual Bethe root contributions. The integration contour and the extra contributions that arise by returning the integration contour for excited states, $C_{e x c}$, to the real line, $C_{c a n}$, are illustrated in the left panel of figure 4.

Analogously, for $Y_{+}$and $Y_{-}$the integration contour needs to be picked essentially in the same manner, with of course the only exception that it should start at -2 and end at 2. In particular, it is chosen in such a way that we pick up both the pole of $Y_{+}$at $u^{-}$and the zero of $1-Y_{-}$at $-u^{-}$, as illustrated in the right panel of figure 4 .

Now we would like to briefly come back to the zeroes of $Y_{M \mid v w}$ in model II. Looking at equation (4.5), we see that they are either real or imaginary depending on a state $(p)$ and the number $M$ under consideration. Also, considered as a function of momentum, a given zero will go from real to imaginary values as the momentum is increased $(0 \leq p \leq \pi)$. When it is real or imaginary but lies between $-i / g$ and the real axis, we need to take its contribution into account in the TBA equations. However, from equation (4.5) we see that as momentum is increased, a given zero will move out of the physical strip $-1 / g \leq \operatorname{Im}(v) \leq 1 / g$ and eventually no longer needs to be taken into account. So the appearance of the zeroes $r_{M}$ in the TBA is state-dependent. Of course, the zeroes also move as $g$ changes, but for now we are concerned only with the small $g$ behaviour just discussed; we refer the reader to appendix B for further details on the behaviour in $g$. 
We would like to note that the kernels and S-matrices appearing below have been defined and are completely listed in [17]; for the sake of brevity we refrain from reproducing the list here and hence refer the reader there for their exact form.

Taking the integration contours as indicated above together with the simplified TBA equations for the ground state derived in $[13,14]$, and returning the integration contours to the real line of the mirror theory, we obtain the excited state TBA equations for a single magnon. For both comparison purposes and brevity, we will discuss model I and model II side by side, splitting the TBA into the always present ground state and $Y_{1}$ contributions, and the extra terms that come from zeroes and poles discussed above which differ between the twisted and untwisted case, see table 2. Please note that in what follows for model II, for completeness we are always writing down the contributions from zeroes $r_{M}$; of course they are not present if the corresponding zero falls outside the lower-half of the physical strip. We then find the following sets of integral equations.

- $M \mid w$-strings; $M \geq 1, Y_{0 \mid w}=0$

$$
V_{M \mid w} \equiv \log Y_{M \mid w}-\underline{\log \left(1+Y_{M-1 \mid w}\right)\left(1+Y_{M+1 \mid w}\right) \star s}-\delta_{M 1} \log \frac{1-\frac{1}{Y_{-}}}{1-\frac{1}{Y_{+}}} \hat{x} s .
$$

For model I we find $V_{M \mid w}=0$, i.e. no driving terms arise and the equations coincide with the ground state ones.

The case of model II requires special care. According to the analytic structure of $Y_{M \mid w}$, a naive guess would be to add the following driving term

$$
V_{M \mid w}=-\underline{\log S\left(\rho_{M-1}^{-}-u\right) S\left(\rho_{M+1}^{-}-u\right)} .
$$

Here the extra contributions arise from the zeroes of $1+Y_{M-1 \mid w}$ and $1+Y_{M+1 \mid w}$, as indicated by the blue underlining; color coding has been used in all equations below for the readers convenience. For $M=1$ the contribution $\log S\left(\rho_{0}^{-}-u\right)$ is due to $Y_{-}\left(\rho_{0}^{-}\right)=1$. These TBA for model II require special care due to state dependence of the analytic properties of the Y-functions. In addition to the roots discussed above, for a given $M$ the (sign of) the asymptotic value of the Y-function on the real mirror line depends on the magnon momentum, hence the state under consideration. As is clear from the form of the roots $(4.8),(4.9)$, a root $\rho_{M}$ moves through infinity for $p=\frac{2 \pi}{M+1}$. Since $Y_{M \mid w}$ has two roots, $\rho_{M-1}$ and $\rho_{M+1}$ and, say $\rho_{M-1}$ always remains finite around $p=\frac{2 \pi}{M+2}$, while $\rho_{M+1}$ moves from positive infinity to negative infinity, it is clear that the sign of the asymptote of $Y_{M \mid w}$ must change, hence being zero at $p=\frac{2 \pi}{M+2}$. Let us illustrate this for the specific example of $Y_{1 \mid w}$, around $p=\frac{2 \pi}{3}$. Depending on the side from which we approach $p=\frac{2 \pi}{3}, \rho_{2}= \pm \infty$, but this merely means that precisely at this point $Y_{1 \mid w}$ asymptotes to zero in both directions. In figure 5 we have plotted $Y_{1 \mid w}$ for $p=\frac{18 \pi}{30}, \frac{2 \pi}{3}$ and $\frac{22 \pi}{30}$ respectively, at $g=1 / 10$. It is clear that $Y_{1 \mid w}$ asymptotes to a positive value in both directions for states with $p<\frac{2 \pi}{3}$, while it asymptotes to zero and negative values for $p=\frac{2 \pi}{3}$ and $p>\frac{2 \pi}{3}$ respectively. 


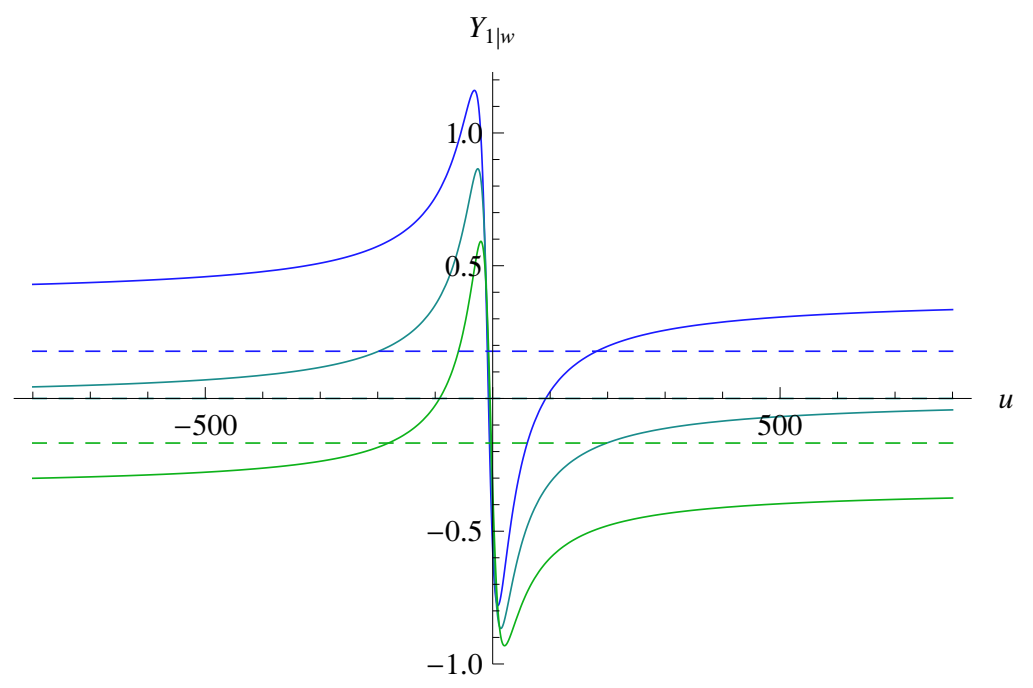

Figure 5. $Y_{1 \mid w}$ on the real mirror line for $p=\frac{18 \pi}{30}, \frac{2 \pi}{3}, \frac{22 \pi}{30}$ from down to up, in green, teal and blue respectively. Their respective asymptotes have been indicated by dashed lines; note the change in the sign of the asymptote as $p$ crosses $\frac{2 \pi}{3}$.

In order to correctly account for the asymptotics of $Y_{M \mid w}$ in the TBA equations, in addition to the standard driving term, for a given state it might be necessary to add $i \pi$ to the right-hand-side of the corresponding equation. Explicitly therefore, the equations with the above driving term should be understood modulo $i \pi$, whose presence can be determined from the sign of the asymptote of the corresponding Y-function.

- $M \mid v w$-strings; $M \geq 1, Y_{0 \mid v w}=0$

$$
\begin{aligned}
V_{M \mid v w} \equiv & \log Y_{M \mid v w}(v) \\
& +\log \left(1+Y_{M+1}\right) \star s-\underline{\log \left(1+Y_{M-1 \mid v w}\right)\left(1+Y_{M+1 \mid v w}\right) \star s} \\
& -\delta_{M 1} \log \frac{1-Y_{-}}{1-Y_{+}} \hat{\star} s,
\end{aligned}
$$

where for the two different models we have

$$
V_{M \mid v w}= \begin{cases}-\underline{\delta_{M 1} \log S\left(u^{-}-v\right)}-\underline{\log S\left(r_{M-1}^{-}-v\right) S\left(r_{M+1}^{-}-v\right)} & \text { I } \\ \left.-\underline{\delta_{M 1} \log S\left( \pm u^{-}-v\right)}-\underline{\log S\left( \pm r_{M-1}^{-}-v\right) S\left( \pm r_{M+1}^{-}\right.}-v\right) & \text { II }\end{cases}
$$

Here and in what follows below we implicitly assume a sum over the \pm sign in front of the zeroes. Given the shape of the integration contour, for $M=1$ the first term in both models comes from the pole of $Y_{+}$at $v=u^{-}$, and the zero of $1-Y_{-}$at $v=-u^{-}$in model II, whereas the second terms come from the zeroes of $1+Y_{M-1 \mid v w}$ and $1+Y_{M-1 \mid v w}$, cf. Table 2. So we see that also at weak coupling, single particle 
states have additional contributions in the TBA equations for $v w$-strings. Finally, we see that the pole of $S\left( \pm u^{-}-v\right)$ cancels the zero of $Y_{1 \mid v w}(v)$ at $v= \pm u$ as it must, and that equation (4.11) is compatible with the reality condition for Y-functions.

- $y$-particles

$$
\begin{aligned}
V_{ \pm} \equiv & \log \frac{Y_{+}}{Y_{-}}(v)+\log S_{1_{*} y}(u, v)-\log \left(1+Y_{Q}\right) \star K_{Q y}=0, \\
V_{\mp} \equiv & \log Y_{-} Y_{+}(v)+\log \frac{\left(S_{x v}^{1_{*} 1}\right)^{2}}{S_{2}} \star s(u, v)-\frac{2 \log \frac{1+Y_{1 \mid v w}}{1+Y_{1 \mid w}} \star s}{{ }^{Q 1} \star s,} \\
& +\log \left(1+Y_{Q}\right) \star K_{Q}+2 \log \left(1+Y_{Q}\right) \star K_{x v}^{Q 1}
\end{aligned}
$$

where for the two different models we have

$$
V_{\mp}= \begin{cases}-2 \log S\left(r_{1}^{-}-v\right) & \mathrm{I} \\ -\underline{2 \log S\left( \pm r_{1}^{-}-v\right)-2 \log S\left(\rho_{1}^{-}-v\right)} & \mathrm{II}\end{cases}
$$

Here the additional contributions come from the term $\log \frac{1+Y_{1 \mid v w}}{1+Y_{1 \mid w}}$ and we have used the following notation

$$
\log \frac{\left(S_{x v}^{1 * 1}\right)^{2}}{S_{2}} \star s(u, v) \equiv \int_{-\infty}^{\infty} d t \log \frac{S_{x v}^{1 * 1}(u, t)^{2}}{S_{2}(u-t)} s(t-v) .
$$

Moreover, $S_{1_{*} y}\left(u_{j}, v\right) \equiv S_{1 y}\left(z_{* j}, v\right)$ is a shorthand notation for the S-matrix with the first and second arguments in the string and mirror regions, respectively. The same convention is used for other S-matrices and kernels.

- $Q$-particles

The TBA equations for $Q$-particles can be cast in particularly convenient form called the hybrid equations; their general form has been derived in [17]. It is here that we find another significant difference from level-matched cases studied previously, as well as between model I and II. We find that in order for the asymptotic Y-functions to satisfy the following integral equations for one-particle states, for model I the length parameter $L_{T B A}$ has to be equal to the angular momentum ${ }^{12} \mathrm{~J}$, whereas in model II we find that $L_{T B A}=J+1$.

This merits a further discussion, as this behaviour is of course far from an accident. We have briefly investigated the hybrid equations for two and three level-unmatched magnons in the untwisted theory, and there we consistently find the relation to be $L_{T B A}=J$. On the other hand, for level-matched states it was observed in [17] that $L_{T B A}=J+2$. Thus, in the untwisted case there is a clear difference between physical (level-matched) and unphysical (level-unmatched) states, rather than a difference

\footnotetext{
${ }^{12}$ In other words, the same as for the ground state [29].
} 
between different numbers of magnons. Doing the twist removes some of the differences between the level-matched and unmatched cases, hence leaving us in some sense somewhere in between ${ }^{13}$ with $L_{T B A}=J+1$. In the equations below we thus have

$$
L_{T B A}=\left\{\begin{array}{lll}
J & \mathrm{I} \text { level-unmatched } \\
J+1 & \mathrm{II} \\
J+2 & \mathrm{I} \text { level-matched }
\end{array}\right.
$$

The hybrid equations for $Q$-particles are now given by

$$
\begin{aligned}
& V_{Q} \equiv \log Y_{Q}(v)+L_{T B A} \widetilde{\mathcal{E}}_{Q}-\log \left(1+Y_{Q^{\prime}}\right) \star\left(K_{\mathfrak{s l}(2)}^{Q^{\prime} Q}-2 s \star K_{v w x}^{Q^{\prime}-1, Q}\right)+\log S_{\mathfrak{s l}(2)}^{1_{*} Q}(u, v) \\
& -\underline{2 \log \left(1+Y_{1 \mid v w}\right) \star s \hat{\star} K_{y Q}}-\underline{2 \log \left(1+Y_{Q-1 \mid v w}\right) \star s}
\end{aligned}
$$

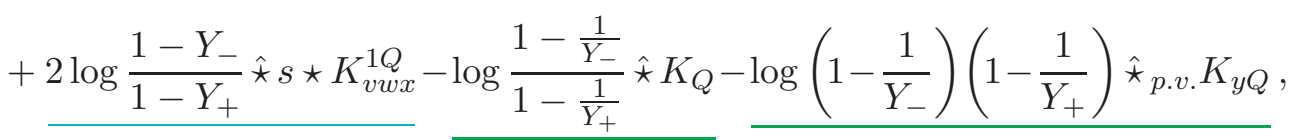

where for model I we have

$$
\begin{aligned}
V_{Q}=- & \frac{2 \log S \hat{\star}_{p . v .} K_{y Q}\left(r_{1}^{-}, v\right)}{-}-\underline{2 \log S\left(r_{Q-1}^{-}, v\right)}+ \\
& +\underline{2 \log S \star_{p . v .} K_{v w x}^{1 Q}\left(u^{-}, v\right)}-\underline{\log S_{v w x}^{1 Q}(u, v)},
\end{aligned}
$$

while for model II we have

$$
\begin{aligned}
V_{Q}=- & \frac{2 \log S \hat{\star}_{p . v .} K_{y Q}\left( \pm r_{1}^{-}, v\right)}{+}-\underline{2 \log S\left( \pm r_{Q-1}^{-}, v\right)}+ \\
+ & \underline{2 \log S \star_{p . v .} K_{v w x}^{1 Q}\left( \pm u^{-}, v\right)} \pm \underline{\log S_{v w x}^{1 Q}( \pm u, v)}+ \\
& -\underline{\log S_{Q}\left(-u^{-}, v\right)}-\underline{\log S_{y Q}\left(-u^{-}, v\right)} .
\end{aligned}
$$

In the above $K_{v w x}^{0, Q}=0$ and $Y_{0 \mid v w}=0$, which implies that the $\log S\left( \pm r_{0}^{-}, v\right)$ terms are also not present. The principal value prescriptions are required due to the pole of $S(v)$ at $v=-i / g$, and are taken in accord with the integration contour $C_{1}$ in the term $\log S \star K_{y Q}$ running slightly above the real line, while for $\log S \star K_{v w x}^{1 Q}$ the contour $C_{2}$ runs slightly above the real line in model I (like $C_{1}$ ) and slightly below and above the negative and positive half real axis in model II respectively, as illustrated in figure 6 . We would like to note that the principal value prescription for the terms involving $K_{y Q}$ give extra contributions, but these precisely cancel against one another. All extra terms above follow from the same reasoning as for the other TBA equations; once more we have explicitly indicated their origin via color coding.

\footnotetext{
${ }^{13}$ We will see more examples of how the twist brings us 'closer' to the level-matched case later.
} 


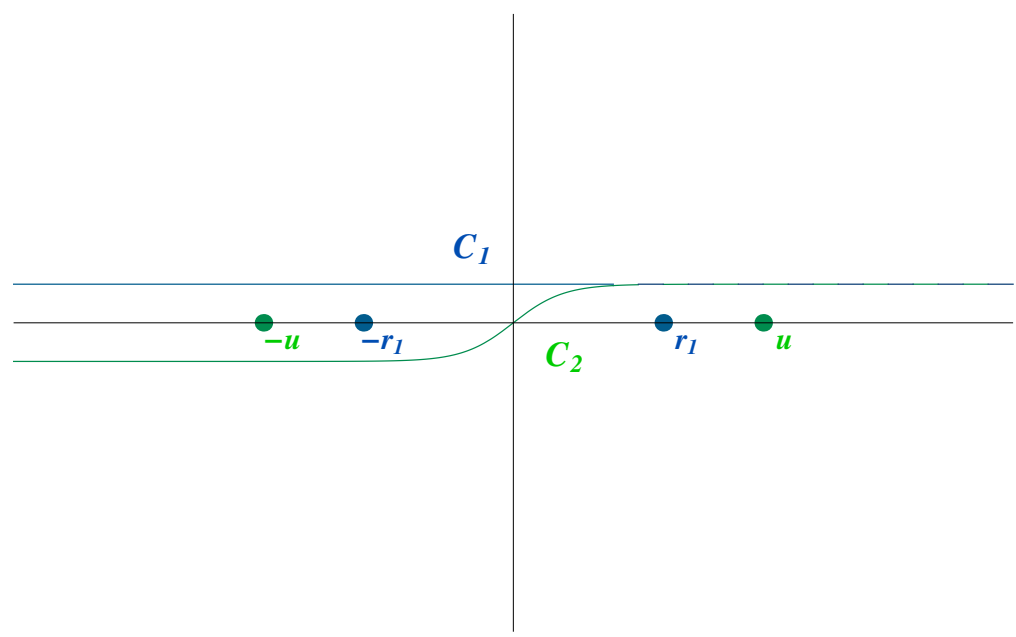

Figure 6. The integration contours for the kernels $\log S \star K_{y Q}$ and $\log S \star K_{v w x}^{1 Q}$ in the hybrid equations.

\subsection{Exact Bethe equations}

Analytically continuing the hybrid equations in the rapidity $v$ to the string region needs to be carefully done as certain terms in the equations below have branch cuts when considered as a function of $v$. This continuation has been carried out in [17] for the Konishi state, and here we follow the same logic to obtain

$$
\begin{aligned}
V_{Q_{*}} & \equiv \pi i(2 n+1)-i L_{T B A} p-\log \left(1+Y_{Q}\right) \star\left(K_{\mathfrak{s l}(2)}^{Q 1_{*}}-2 s \star K_{v w x}^{Q-1,1_{*}}\right)+\log S_{\mathfrak{s l}(2)}^{1_{*} 1_{*}}(u, u) \\
& -\frac{2 \log \left(1+Y_{1 \mid v w}\right) \star\left(s \hat{\star} K_{y 1_{*}}+\tilde{s}\right)}{2}+\frac{\log \frac{1-Y_{-}}{1-Y_{+}} \hat{\star} s \star_{p . v .} K_{v w x}^{11_{*}}+2 \log \frac{1-Y_{-}}{1-Y_{+}} \hat{\star} s}{-}-\underline{\log \frac{1-\frac{1}{Y_{-}}}{1-\frac{1}{Y_{+}}} \hat{\star} K_{1}-}-\underline{\log \left(1-\frac{1}{Y_{-}}\right)\left(1-\frac{1}{Y_{+}}\right) \hat{\star} K_{y 1_{*}}}
\end{aligned}
$$

where for model I we have

$$
\begin{aligned}
V_{Q_{*}}=- & \frac{2 \log S \hat{\star}_{p . v .} K_{y 1_{*}}\left(r_{1}^{-}, u\right)}{2}-\underline{2 \log S\left(r_{1}^{-}-i / g-u\right)} \\
& +\underline{2 \log \operatorname{Res}(S) \star K_{v w x}^{11_{*}}\left(u^{-}, u\right)}-\underline{2 \log \frac{2 i}{g} \frac{x^{-}-\frac{1}{x^{-}}}{x^{+}-x^{-}}},
\end{aligned}
$$

and for model II we have

$$
\begin{aligned}
V_{Q_{*}}=- & \frac{2 \log S \hat{\star}_{p . v .} K_{y 1_{*}}\left( \pm r_{1}^{-}, u\right)}{-}-\underline{2 \log S\left( \pm r_{1}^{-}-i / g-u\right)}+ \\
+ & \underline{2 \log \operatorname{Res}(S) \star K_{v w x}^{11_{*}}\left(u^{-}, u\right)}-\frac{2 \log \frac{2 i}{g} \frac{x^{-}-\frac{1}{x^{-}}}{x^{+}-x^{-}}}{} \\
+ & \underline{2 \log S \star_{p . v .} K_{v w x}^{11_{*}}\left(-u^{-}, u\right)}+\underline{\log S_{v w x}^{11_{*}}(-u, u)}+\underline{\log S\left(-u^{-}, u\right)}+ \\
& -\underline{\log S_{1}\left(-u^{-}, u\right)}-\underline{\log S_{y 1_{*}}\left(-u^{-}, u\right) .}
\end{aligned}
$$


In the above we use the notations

$$
\begin{aligned}
\log \operatorname{Res}(S) \star K_{v w x}^{11_{*}}\left(u^{-}, v\right) & =\int_{-\infty}^{+\infty} \mathrm{d} t \log \left[S\left(u^{-}-t\right)(t-u)\right] K_{v w x}^{11 *}(t, v), \\
\tilde{s}(u) & =s\left(u^{-}\right),
\end{aligned}
$$

the momentum of the magnon is $p=i \widetilde{\mathcal{E}}_{Q}\left(z_{*}\right)=-i \log \frac{x_{s}\left(u+\frac{i}{g}\right)}{x_{s}\left(u-\frac{i}{g}\right)}$, and the second argument in all kernels in (4.16) is the Bethe root $u$.

For a single magnon, the exact Bethe equation in the $\mathfrak{s l}(2)$ sector can be written in the form

$$
\pi i(2 n+1)=i J p-\log S_{\mathfrak{s l}(2)}^{1 * 1 *}(u, u)+i \mathcal{R}
$$

where $\mathcal{R}$ encodes a correction to the asymptotic Bethe equation represented by the remaining terms. For asymptotic solution we should find $\mathcal{R}^{\text {asym }}=0$, which also serves as a non-trivial check of the correctness of the analytic continuation procedure.

Discarding for large $J$ the exponentially small $Y_{Q}$-functions in the right hand side of equation (4.16) and subtracting from equation (4.16) the contribution of the asymptotic Bethe equation, we find $\mathcal{R}^{\text {asym }}$ for models I and II. We recall that the difference between $L_{T B A}$ and $J$ that enters $\mathcal{R}^{\text {asym }}$ differs between the models; $L_{T B A}-J=0$ for model I and $L_{T B A}-J=1$ for model II. Substituting the asymptotic Y-functions into the respective $\mathcal{R}^{\text {asym }}$, we find that the corresponding quantity perfectly vanishes for both models.

\section{Finite-size corrections to the dispersion: model I}

For a fixed $J$ and small $g$, the $Y_{Q}$-functions are small. The finite-size correction term then provides the so-called wrapping correction to the energy, or, equivalently, to the scaling dimension of a gauge theory operator at weak coupling. The goal of the next two sections is to compute the leading wrapping corrections to the asymptotic energy and momentum of a single magnon for models I and II.

When expanding energy and momentum for small $g$ around the asymptotic solutions $Y_{Q}^{o}$, we obtain the leading order corrections to the energy and momentum

$$
E_{\mathrm{LO}}=-\frac{1}{2 \pi} \sum_{Q=1}^{\infty} \int \mathrm{d} v Y_{Q}^{o}(v), \quad P_{\mathrm{LO}}=-\frac{1}{2 \pi} \sum_{Q=1}^{\infty} \int \mathrm{d} v \frac{2 v}{Q^{2}+v^{2}} Y_{Q}^{o}(v)
$$

We recall that formula (5.1) for the energy follows from Lüscher's perturbative approach and it was shown to successfully describe the four-loop corrections to the anomalous dimension for twist two operators $[4,7]$. When the level-matching condition is imposed, the functions $Y_{Q}^{o}(v)$ are symmetric with respect to $v \rightarrow-v$ and therefore, $P_{\mathrm{LO}}$ vanishes, as it should. For a single magnon however, the $Y_{Q}^{o}(v)$ are no longer symmetric which leads to non-trivial values of $P_{\mathrm{LO}}$. 


\subsection{LO wrapping correction to the energy and momentum}

In model I we deal with periodic boundary conditions for all fields (no twist). Using the untwisted transfer matrices $T_{Q, 1}$, we find that in the limit $g \rightarrow 0$ the $Y_{Q}$-functions associated to a single magnon with rapidity $u$ are

$$
Y_{Q}^{o}(v)=\frac{g^{2 J}}{\left(v^{2}+Q^{2}\right)^{J}} \frac{4 Q^{2}\left(u-\sqrt{u^{2}+1}\right)^{2}(v-u)^{2}}{\left[(Q-1)^{2}+(v-u)^{2}\right]\left[(Q+1)^{2}+(v-u)^{2}\right]} .
$$

The energy integral (5.1) can be computed by residues for any fixed value of $J$ and the first wrapping correction to the asymptotic dispersion relation is then found by taking the sum over the bound state number $Q$.

In appendix $\mathrm{C}$ we present the full result of the leading order wrapping correction depending on the rapidity $u$. Here we present only the result upon substituting the solution of the Bethe equations $e^{i p J}=1$. We find for model I the following compact result for $J>4$

$$
E_{\mathrm{LO}}^{\mathrm{I}}(J)=g^{2 J} \sum_{m=1}^{\left\lfloor\frac{J}{2}\right\rfloor+1} C_{m}(J) \zeta(2 J-2 m-1) .
$$

Here the coefficients $C_{m}(J)$, written in terms of particle momentum, take the form

$$
\begin{aligned}
& C_{m}(J)=\frac{(-1)^{m+1} \Gamma(2 J-2 m)}{2^{2 J-2 m-1} \Gamma(J) \Gamma(J-2 m+2)} \tan ^{2} \frac{p}{4}\left(\sin \frac{p}{2}\right)^{2 m-1} \times \\
& \times\left[(m-1) \sin \frac{p}{2} \cos m p-(J-m) \cos \frac{p}{2} \sin m p\right] .
\end{aligned}
$$

The above formula does not hold for $J \leq 4$ because of divergent terms. However, for these values of $J$ the wrapping corrections are easily found to be

$$
\begin{aligned}
& E_{\mathrm{LO}}^{\mathrm{I}}(4)=2 \zeta(3) \sin ^{6} \frac{p}{4} \cos ^{2} \frac{p}{4}(2+4 \cos p+\cos 2 p)-\frac{5}{4} \zeta(5) \sin ^{4} \frac{p}{4}(1+\cos p) \\
& E_{\mathrm{LO}}^{\mathrm{I}}(3)=-\frac{3}{16} \zeta(3) \sin ^{2} p \tan ^{2} \frac{p}{4} \\
& E_{\mathrm{LO}}^{\mathrm{I}}(2)=\sin ^{4} \frac{p}{4} \cos p .
\end{aligned}
$$

It is worth stressing that for generic $p$ the integral for $E_{\mathrm{LO}}^{\mathrm{I}}(J)$ also gives rise to polygamma functions. However, these cancel out on solutions of the Bethe equation leaving only a sum of $\zeta$-functions.

We finish this discussion by mentioning one important subtlety concerning the levelmatching condition. As we have seen from the single magnon example and as can be verified for the case of several particles, without level matching imposed the wrapping correction in model I expands at weak coupling as

$$
E_{\mathrm{LO}}^{\mathrm{I}}=g^{2 J} E^{(2 J)}+\ldots=g^{2 L_{T B A}} E^{\left(2 L_{T B A}\right)}+\ldots,
$$

since, as we have shown in section 3 , in the level-unmatched case $L_{T B A}=J$. On the other hand, with the level matching imposed, one finds that the leading term of order $g^{2 J}$ cancels 
out $^{14}$ and the expansion of the wrapping corrections starts from $g^{2 J+4}$. However, as we have seen, for the level-matched case the TBA equations require to set up $L_{T B A}=J+2$. Thus,

$$
E_{\mathrm{LO}}^{\mathrm{I}}=g^{2 J+4} E^{(2 J+4)}+\ldots=g^{2 L_{T B A}} E^{\left(2 L_{T B A}\right)}+\ldots,
$$

i.e. in both cases the expansion starts from $g^{2 L_{T B A}}$.

Now we present the leading finite size correction to the momentum. To make the relation to $E_{\mathrm{LO}}$ more transparent, we introduce

$$
E_{\mathrm{LO}}^{\mathrm{I}}=\frac{\left(u-\sqrt{u^{2}+1}\right)^{2}}{2 \pi} \mathscr{E}, \quad \quad P_{\mathrm{LO}}^{\mathrm{I}}=\frac{\left(u-\sqrt{u^{2}+1}\right)^{2}}{2 \pi} \mathscr{P} .
$$

From the identity

$$
\partial_{u} \frac{Y_{Q}^{0}(v)}{\left(u-\sqrt{u^{2}+1}\right)^{2}}+\frac{2 J v}{v^{2}+Q^{2}} \frac{Y_{Q}^{0}(v)}{\left(u-\sqrt{u^{2}+1}\right)^{2}}+\frac{\partial_{v} Y_{Q}^{0}(v)}{\left(u-\sqrt{u^{2}+1}\right)^{2}}=0,
$$

it is then easily shown that

$$
J \mathscr{P}=-\partial_{u} \mathscr{E} .
$$

It is now straightforward to find the exact expression for the wrapping corrected momentum by differentiating the formula (C.4). Finally, we point out that formula (5.12) implies the following relation between the leading corrections to the energy and momentum

$$
J P_{\mathrm{LO}}^{\mathrm{I}}=2 \sin \frac{p}{2}\left(\sin \frac{p}{2} \frac{\partial}{\partial p} E_{\mathrm{LO}}^{\mathrm{I}}-E_{\mathrm{LO}}^{\mathrm{I}}\right) .
$$

We would like to note that on solutions of the Bethe equations, the expression for $P_{\mathrm{LO}}^{\mathrm{I}}$ generically contains polygamma terms.

\subsection{Quantization of momentum and exact Bethe equation}

To test the equivalence of the two quantization conditions (3.7), we now need to independently compute the leading finite-size correction to the asymptotic Bethe equations. We will do this analytically by applying the method developed in $[22,25]$. It is based on finding a connection between $Y_{Q}^{o}$ and the transfer matrix of the Heisenberg spin chain. For a single excitation with rapidity $u$ the transfer matrix of the Heisenberg model is

$$
t_{M}(v)=(M+1)(v-u) .
$$

It is readily checked that it satisfies the equation

$$
t_{M}(v+i) t_{M}(v-i)=t_{M+1}(v) t_{M-1}(v)+t_{0}(v+(M+1) i) t_{0}(v-(M+1) i) .
$$

The corresponding Y-function is then defined as

$$
y_{M}(v)=\frac{t_{M+1}(v) t_{M-1}(v)}{t_{M}(v+i) t_{M}(v-i)-t_{M+1}(v) t_{M-1}(v)}=\frac{M(M+2)(v-u)^{2}}{(M+1)^{2}+(v-u)^{2}},
$$

\footnotetext{
${ }^{14}$ The transfer matrix $T_{Q, 1}$ is constant as $g \rightarrow 0$ in the level-unmatched case and behaves as $g^{2}$ when the level matching is imposed.
} 
which, in turn, satisfies the functional equation

$$
y_{M}(v+i) y_{M}(v-i)=\left[1+y_{M+1}(v)\right]\left[1+y_{M-1}(v)\right] .
$$

Obviously, each $y_{M}(v)$ has a double zero at $r_{M}=u$. Furthermore, $y_{M}(v)$ also obeys the normalization condition

$$
y_{M}(u-i)=-1 \text {. }
$$

In terms of the quantities introduced above, the leading order of $Y_{Q}^{o}(v)$ can be conveniently written as

$$
Y_{Q}^{o}(v)=-\frac{4 g^{2 J}}{\left(v^{2}+Q^{2}\right)^{J}}\left(\sqrt{1+u^{2}}-u\right)^{2} j_{Q}
$$

with

$$
j_{Q}=\frac{t_{Q-1}(v)^{2}}{t_{0}(v+(Q+1) i) t_{0}(v+(Q-1) i) t_{0}(v-(Q-1) i) t_{0}(v-(Q+1) i)} .
$$

Moreover, expanding $Y_{M \mid v w}^{o}(v)$ at weak coupling, we find that the leading term coincides with $y_{M}(v)$. This is all that is needed to repeat the computation of $[22,25]$ for the case at hand.

Following the same strategy as employed in [21], we employ the exact Bethe equations in their hybrid form (4.16). These equations will allow us to find the leading finite-size correction $\delta u$ to the asymptotic magnon rapidity $u \equiv u(p)$, the latter given by formulae (3.3) and (3.4). Expanding (4.16) around its asymptotic solution, we get

$$
2 \pi n=J p+\delta \mathcal{R}+\mathcal{O}\left(g^{2 J+1}\right)
$$

Here $\delta \mathcal{R}$ is a leading correction of order $g^{2 J}$ to the asymptotic value of $\mathcal{R}$, the latter being identically zero. As in the two-particle case [21], the term $\delta \mathcal{R}$ is naturally split into three parts

$$
\delta \mathcal{R}=\delta \mathcal{R}^{(1)}+\delta \mathcal{R}^{(2)}+\delta \mathcal{R}^{(3)}
$$

where

$$
\begin{aligned}
& \delta \mathcal{R}^{(1)}=\frac{1}{\pi} \sum_{Q=1}^{\infty} \int \mathrm{d} v Y_{Q}^{o}(v) \frac{v-u}{(Q+1)^{2}+(v-u)^{2}}, \\
& \delta \mathcal{R}^{(2)}=\frac{1}{\pi} \sum_{Q=1}^{\infty} \int \mathrm{d} v Y_{Q+1}^{o}(v)\left\{\mathcal{F}_{Q}(v-u)-\frac{v-u}{Q^{2}+(v-u)^{2}}\right\} .
\end{aligned}
$$

The function $\mathcal{F}_{Q}$ is defined as

$$
\mathcal{F}_{Q}(v)=-\frac{i}{4}\left\{\psi\left(\frac{Q+i v}{4}\right)-\psi\left(\frac{Q-i v}{4}\right)-\psi\left(\frac{Q+2+i v}{4}\right)+\psi\left(\frac{Q+2-i v}{4}\right)\right\} .
$$


The third and most complicated piece is computed in the same way as in [25] by exploiting the relation to the Heisenberg model discussed above. Here we only quote the corresponding result

$$
\delta \mathcal{R}^{(3)}=\frac{1}{\pi} \sum_{Q=1}^{\infty} \int_{-\infty}^{\infty} \mathrm{d} v Y_{Q+1}^{o}(v)\left\{-\mathcal{F}_{Q}(v-u)+\frac{(v-u)^{2}-Q^{2}}{(v-u)\left((v-u)^{2}+Q^{2}\right)}\right\} .
$$

Finally, summing the corrections up, one can show that the resulting expression equals to

$$
\delta \mathcal{R}=\frac{1}{2 \pi} \sum_{Q=1}^{\infty} \int_{-\infty}^{\infty} \mathrm{d} v \frac{g^{2 J}}{\left(v^{2}+Q^{2}\right)^{J}} \frac{\partial}{\partial v}\left[\frac{T_{Q, 1}^{\mathfrak{s l}(2)}(v \mid u)^{2}}{S_{0}(v, u)}\right],
$$

which is in agreement with the proposal by [5]. The quantities $T_{Q, 1}(v \mid u)$ and $S_{0}(v, u)$ are given in appendix $\mathrm{A}$. The expression for $\delta \mathcal{R}$ can be rewritten as

$$
\delta \mathcal{R}=-\frac{1}{2 \pi} \sum_{Q=1}^{\infty} \int_{-\infty}^{\infty} \mathrm{d} v\left[\frac{d Y_{Q}^{o}(v)}{d v}+J \frac{2 v}{Q^{2}+v^{2}} Y_{Q}^{o}(v)\right]=J P_{\mathrm{LO}}
$$

Thus, at leading order the corrected Bethe Ansatz equation takes the form

$$
\frac{2 \pi n}{J}=p+P_{\mathrm{LO}}(p)
$$

This provides a strong argument for compatibility of the exact Bethe equations with the definition of the exact, i.e. non-asymptotic momentum. Equation (5.29) can be solved perturbatively by assuming that the asymptotic value of $p$ is shifted $p \rightarrow p+g^{2 J} \delta p$ but we will not pursue finding an actual solution any further. Rather, we will continue with model II.

\section{Finite-size corrections to the dispersion: model II}

In this section we will discuss both the leading and the next-to-leading order finite-size (wrapping) correction to the energy in model II.

\subsection{LO wrapping correction to the energy and momentum}

In model II the periodicity conditions for bosons are twisted by the total momentum of a state; $\alpha=p / 2$. Using the corresponding twisted transfer matrices one finds that for a single excitation in the limit $g \rightarrow 0$ the leading term of $Y_{Q}^{o}(v)$ is given by

$$
Y_{Q}^{o}(v)=\frac{g^{2 J+4}}{\left(v^{2}+Q^{2}\right)^{J+2}} \frac{16}{\left(1+u^{2}\right)^{2}} \frac{Q^{2}\left(Q^{2}-1+v^{2}-u^{2}\right)^{2}}{\left[(Q-1)^{2}+(v-u)^{2}\right]\left[(Q+1)^{2}+(v-u)^{2}\right]} .
$$

The expansion of $Y_{Q}^{o}$ starts at order $g^{2 J+4}$, which also defines the lowest order of the corresponding wrapping correction to the Bethe Ansatz energy. Performing a similar computation of $Y_{Q}^{o}$ for the multi-particle case, one also finds that in both level-matched and level-unmatched cases the expansion of $Y_{Q}^{o}$ starts from the same order $g^{2 J+4}$. This behavior should be contrasted with the one for $Y_{Q}^{o}$-functions in model I based on the untwisted 
transfer matrix. There we found that for the level-unmatched case the corresponding expansion starts at lower order $g^{2 J}$, irrespective of the number of particles being even or odd, while for the level-matched case the leading term is of order $g^{2 J+4}$.

In this context it is interesting to recall that in [8] an analytic continuation of the leading wrapping correction from even to odd number of particles was proposed. This continuation ${ }^{15}$ amounts to modifying the (untwisted) transfer matrix $T_{Q, 1}$ in such a way (by multiplying certain eigenvalues of the corresponding monodromy matrix by extra factors of $i$ ) that in the level-unmatched case for odd number of particles the expansion of the wrapping correction starts starts from $g^{2 J+4}$, i.e. as for physical states. This modification was proposed to describe an unphysical magnon with momentum $p=\pi$. It is easy to see that our construction of the twisted transfer matrix agrees and correctly generalizes the proposal [8] for the case of an off-shell theory with any number of excited states and for any value of the coupling constant. ${ }^{16}$

For a generic rapidity $u$, the resulting wrapping corrections are given in appendix C. Here we present the wrapping correction on solutions of the Bethe equations. Similar to model I, we find that upon substituting the solution of the Bethe equation $u=\cot \frac{\pi n}{J}$, the contribution of polygamma-functions in the energy correction completely drops out. This again results in a compact form of the wrapping correction, given solely in terms of $\zeta$-functions

$$
E_{\mathrm{LO}}^{\mathrm{II}}(J)=g^{2 J+4}\left[\sum_{m=1}^{\left\lfloor\frac{J}{2}\right\rfloor+1} C_{m}(J) \zeta(2 J-2 m+1)-8 \sin ^{4} \frac{p}{2} \frac{\Gamma\left(J+\frac{3}{2}\right)}{\Gamma(J+2)} \frac{\zeta(2 J+1)}{\sqrt{\pi}}\right] .
$$

Here the coefficients $C_{m}(J)$ written in terms of particle momentum take the form

$$
\begin{aligned}
C_{m}(J)=(-1)^{m} \frac{16}{\sqrt{\pi}} \frac{\Gamma(J-m+1) \Gamma\left(J-m+\frac{3}{2}\right)}{\Gamma(J+2) \Gamma(J-2 m+2)} \sin ^{2 m+3}\left(\frac{p}{2}\right) \times \\
\\
\times\left[m \sin \left(m+\frac{1}{2}\right) p-(J+1) \cos \frac{p}{2} \sin m p\right] .
\end{aligned}
$$

The above formula holds for $J>2$ as for $J=2$ it contains a divergent term originating from $\zeta(1)$. For this case $E_{\mathrm{LO}}^{\mathrm{II}}(2)$ can be computed directly and the result is

$$
E_{\mathrm{LO}}^{\mathrm{II}}(2)=2 \sin ^{6} \frac{p}{2}(2+\cos p) \zeta(3)-\frac{5}{2} \sin ^{4} \frac{p}{2} \zeta(5)
$$

It is readily seen that for $p=0$ the wrapping correction vanishes.

\footnotetext{
${ }^{15}$ Further subtleties concerning the analytic continuation from even to odd number of particles have been discussed in $[48,66]$, where it was argued that the proposal by [8] corresponds to one (-) of the two possible prescriptions for analytic continuation of the harmonic sums with negative values of indices arising on the gauge theory side.

${ }^{16}$ The twist must necessarily be $p$-dependent. Indeed, if one admits that in the single magnon case the constant twist in [8] is valid not only for $p=\pi$ but rather for all values of $p$, then for the physical state with $p=0$ one finds a non-vanishing energy correction which is nonsensical because the corresponding state being the susy descendant of the BPS vacuum is not renormalized.
} 
Next we present the leading finite size correction to the momentum. From the identity

$$
\partial_{v} Y_{Q}^{o}(v)+\partial_{u} Y_{Q}^{o}(v)+\frac{2 v(J+2)}{Q^{2}+v^{2}} Y_{Q}^{o}(v)+\left[\frac{4 u}{1+u^{2}}-\frac{4(v-u)}{Q^{2}-1+v^{2}-u^{2}}\right] Y_{Q}^{o}(v)=0,
$$

it is easily shown that

$$
(J+2) P_{\mathrm{LO}}^{\mathrm{II}}=-\partial_{u} E_{\mathrm{LO}}^{\mathrm{II}}-\frac{4 u}{1+u^{2}} E_{\mathrm{LO}}^{\mathrm{II}}-4 \mathcal{I}_{1},
$$

where

$$
\mathcal{I}_{1}(J)=\frac{1}{2 \pi} \sum_{Q=1}^{\infty} \int d v \frac{v-u}{Q^{2}-1+v^{2}-u^{2}} Y_{Q}^{0}(v) .
$$

The integral $\mathcal{I}_{1}$ can be computed explicitly and it is given in appendix D.2.

Using the explicit result for $\mathcal{I}_{1}$, it is now straightforward to find the expression for the wrapping corrected momentum by differentiating the wrapping correction to the energy. We define the coefficients $D_{m}(J)$ to be

$$
\begin{aligned}
D_{m}(J)= & \frac{2^{2 m-2 J+3}(-1)^{m} \Gamma(2 J-2 m+2)}{\Gamma(J+3) \Gamma(J-2 m+2)}\left(\sin \frac{p}{2}\right)^{2 m+4} \\
& \left((J-m+1)(2 m+1) \sin (n+1) p \sin \frac{p}{2}-2(J+2) m \sin \frac{p}{2} \cos \left(n+\frac{1}{2}\right) p\right),
\end{aligned}
$$

and then $P_{\mathrm{LO}}^{\mathrm{II}}$ becomes

$$
\begin{aligned}
P_{\mathrm{LO}}^{\mathrm{II}}=\frac{\left(\sin \frac{p}{2}\right)^{J+2}}{2^{J-1}} & {\left[\frac{\sin \frac{J p}{2} A(J+2)}{4 i^{2-J}(J+2) !}+\frac{\sin \frac{(J-1) p}{2} \sin \frac{p}{2} A(J+1)}{2 i^{1-J}(J+1) !}+\frac{\sin \frac{(J+2) p}{2} \sin ^{2} \frac{p}{2} A(J)}{i^{-2-J} J !}\right.} \\
& \left.+2 \frac{\sin \frac{(J+1) p}{2} \sin ^{3} \frac{p}{2} A(J-1)}{i^{-3-J}(J-1) !}\right]-\sum_{m=0}^{\left\lfloor\frac{J+1}{2}\right\rfloor} D_{m}(J) \zeta(2 J-2 m+1),
\end{aligned}
$$

where $A(J)$ is defined in equation (C.9). Notice that, in contrast to the wrapping correction to the energy, the polygamma functions here do not cancel on a solution of the Bethe equation.

\subsection{Quantization of momentum and exact Bethe equation}

Once again, following $[22,25]$, it is possible to establish a connection between the leading order of $Y_{Q}^{o}$ and the transfer matrix of the Heisenberg spin chain. For a single excitation with rapidity $u$ we define the following transfer matrix

$$
t_{Q}(v)=(Q+1)\left(Q(Q+2)+v^{2}-u^{2}\right) .
$$

It is readily checked that it satisfies the equation

$$
t_{Q}(v+i) t_{Q}(v-i)=t_{Q+1}(v) t_{Q-1}(v)+t_{0}(v+(Q+1) i) t_{0}(v-(Q+1) i) .
$$

The associated Y-functions are again defined as

$$
y_{Q}(v)=\frac{t_{Q+1}(v) t_{Q-1}(v)}{t_{Q}(v+i) t_{Q}(v-i)-t_{Q+1}(v) t_{Q-1}(v)} .
$$


Explicitly,

$$
y_{Q}(v)=Q(Q+2) \frac{\left[Q^{2}-1+v^{2}-u^{2}\right]\left[(Q+2)^{2}-1+v^{2}-u^{2}\right]}{\left[(Q+1)^{2}+(v-u)^{2}\right]\left[(Q+1)^{2}+(v+u)^{2}\right]} .
$$

These functions satisfy the functional relation

$$
y_{Q}(v+i) y_{Q}(v-i)=\left[1+y_{Q+1}(v)\right]\left[1+y_{Q-1}(v)\right] .
$$

Obviously, each $y_{Q}(v)+1$ has four roots $\pm u_{Q} \pm i$, that is

$$
y_{Q}\left( \pm u_{Q} \pm i\right)=-1,
$$

where $u_{Q}=\sqrt{u^{2}-Q^{2}-2 Q}$.

In terms of the quantities introduced above, the leading order of $Y_{Q}^{o}(v)$ can be conveniently written as

$$
Y_{Q}^{o}(v)=\frac{16 g^{2 J+4}}{\left(v^{2}+Q^{2}\right)^{J+2}} \frac{j_{Q}}{\left(1+u^{2}\right)^{2}}
$$

with

$$
j_{Q}=\frac{t_{Q-1}(v)^{2}}{\tau(v+(Q+1) i) \tau(v+(Q-1) i) \tau(v-(Q-1) i) \tau(v-(Q+1) i)},
$$

where

$$
\tau(v)=v-u .
$$

Expanding $Y_{M \mid v w}^{o}$-functions at weak coupling, we find $Y_{M \mid v w}^{o}(v)=y_{M}(v)$. We would like point out that curiously enough $y_{M}$ coincides precisely with the leading term in the expansion of $Y_{M \mid v w}^{o}$ for the case of two particles with the level-matching condition imposed, cf. equation (3.11) in [17]; in particular, each $y_{M}$ has four zeroes at weak coupling. Furthermore, the transfer matrix $t_{Q}(v)$ given by equation (6.10) which we used to express the asymptotic Y-functions for a single and, therefore, unphysical magnon, coincides with the two-particle transfer matrix of the Heisenberg model

$$
t_{Q}^{2 p t}=(Q+1)\left[Q(Q+2)+\left(v-u_{1}\right)\left(v-u_{2}\right)\right]
$$

with the level-matching condition $u_{2}=-u_{1}$ imposed.

Using this relationship with the Heisenberg model, one can repeat the computation of the leading correction to the asymptotic Bethe equations. This time we find

$$
\delta \mathcal{R}=\frac{1}{\pi} \sum_{Q=1}^{\infty} \int \mathrm{d} v\left[\frac{v-u}{(Q-1)^{2}+(v-u)^{2}}+\frac{v-u}{(Q+1)^{2}+(v-u)^{2}}-\frac{v+u}{Q^{2}-1+v^{2}-u^{2}}\right] Y_{Q}^{o} .
$$

The relation with to $P_{\mathrm{LO}}$ is

$$
\delta \mathcal{R}=(J+2) P_{\mathrm{LO}}^{\mathrm{II}}+2 \mathcal{I}_{1} .
$$

Thus, for model II the leading correction to the asymptotic Bethe equations does not agree with the corresponding correction to the momentum, as defined by equation (3.6). This result suggests that in a twisted theory the expression for the actual momentum requires a modification of expression (3.6) in order to agree with the correction to the Bethe ansatz. It would be interesting to find its non-perturbative definition. 


\subsection{Comparing energies of $\mathfrak{s l}(2)$ and $\mathfrak{s u}(2)$ states}

It turns out that the momentum twisted versions of the $\mathfrak{s l}(2)$ and $\mathfrak{s u}(2)$ transfer matrix are related in a very direct manner. In the $\mathfrak{s u}(2)$ sector we will denote the different momentum twists by + and - in each factor, writing $T_{Q, 1}^{\mathfrak{s u}(2) \pm}$ for a transfer matrix twisted with $\alpha=$ $\pm p / 2$. As discussed before, for $\mathfrak{s l}(2)$ this distinction is not needed as the direction of the twist is inconsequential and we will simply write $T_{Q, 1}^{\mathfrak{s l}(2)}$. Expanding the transfer matrices at weak coupling, one finds at leading order the following very simple relations

$$
T_{Q, 1}^{\mathfrak{s l}(2)}=\left(\frac{g^{2}}{Q^{2}+u^{2}}\right)^{\mp \frac{1}{2}} T_{Q, 1}^{\mathfrak{s u}(2) \pm} .
$$

Now we know that a similar prefactor occurs in the expansion of the $Y_{Q}$-function (2.17),

$$
Y_{Q}=\left(\frac{g^{2}}{Q^{2}+u^{2}}\right)^{J} T^{\ell} T^{r} S_{\mathfrak{s l}(2)}^{Q 1_{*}}+\ldots,
$$

which means that we can effectively absorb this prefactor in a shift of $J$ depending on our choice of twists. In order to indicate this clearly, we write $Y_{Q}^{\mathfrak{s l}(2)}$ for the momentum twisted Y-function in the $\mathfrak{s l}(2)$ sector, and $Y_{Q}^{\mathfrak{s u}(2) \pm \pm}$ for the Y-function in the $\mathfrak{s u}(2)$ sector, where the twists are taken $\pm p / 2$ in the left and right factor, respectively. We then find the relations

$$
Y_{Q}^{\mathfrak{s l}(2)}(J)=Y_{Q}^{\mathfrak{s u}(2) \pm \mp}(J)=Y_{Q}^{\mathfrak{s u}(2)++}(J+1)=Y_{Q}^{\mathfrak{s u}(2)--}(J-1) .
$$

Indicating the twists in differently twisted theories by subscript signs, these relations imply that the LO wrapping corrections to the energy of the gauge theory operators of the schematic form $\left(\gamma^{n} D Z^{J}\right),\left(\gamma_{ \pm \mp}^{n} \Phi Z^{J}\right)$, and $\left(\gamma_{ \pm \pm}^{n} \Phi Z^{J \pm 1}\right)$ all coincide. Here $\gamma$ is an element of a representation of the orbifold group, appropriate for the field-theoretic realization of the twist, and the integer $n$ labels the $n$-th twisted sector in the orbifold theory. Finally, the twists ++ and -- also affect the $\mathfrak{s u}(2)$ Bethe equations, cf. equation (2.22). Now we immediately see that these twists can be effectively compensated by shifting $J \rightarrow J \pm 1$, leading to the one and the same Bethe equation $e^{i p J}=1$. Therefore, the theories obtained by doing the various twists are directly related by a simple shift in $J$ in the $\mathfrak{s u}(2)$ sector.

Finally we would like to note that there exists a curiously simple relationship not only between the leading order but also the full twisted transfer matrices $T_{Q, 1}^{\mathfrak{s u}(2) \pm}$ and $T_{Q, 1}^{\mathfrak{s l}(2)}$. Namely,

$$
T_{Q, 1}^{\mathfrak{s u}(2)+} T_{Q, 1}^{\mathfrak{s u}(2)-}=T_{Q, 1}^{\mathfrak{s}(2)^{2}}
$$

An analogous relation holds in the Konishi case, where it was observed that for level matched states $T_{Q, 1}^{\mathfrak{s l}(2)}$ and $T_{Q, 1}^{\mathfrak{s u}(2)}$ coincide [17]. Of course this agrees with the above relation, since for level matched states the twist is trivial. ${ }^{17}$

\footnotetext{
${ }^{17}$ For a single magnon the level matched equivalence is completely trivial.
} 


\subsection{NLO wrapping correction to the energy}

To describe the next-to-leading order wrapping correction all quantities in (3.1) must be carefully expanded to two orders higher in $g$. In what follows we will discuss the expansion of the different terms separately and give their contribution to the energy.

First we consider the term in the integral that is not related to the Y-function

$$
\frac{d \tilde{p}^{Q}}{d v}=1+2 g^{2} \frac{v^{2}-Q^{2}}{\left(v^{2}+Q^{2}\right)^{2}}+\mathcal{O}\left(g^{4}\right) .
$$

This obviously gives rise to the contribution

$$
E_{\mathrm{NLO}}^{(1)} \equiv 2 g^{2} \sum_{Q=1}^{\infty} \int d v \frac{v^{2}-Q^{2}}{\left(v^{2}+Q^{2}\right)^{2}} Y_{Q}^{0}(v) .
$$

This term can be written as the sum of two terms

$$
E_{\mathrm{NLO}}^{(1)}(J)=\frac{\mathcal{I}_{2}(J)}{\left(u^{2}+1\right)^{2}}-2 E_{\mathrm{LO}}^{\mathrm{II}}(J+1)
$$

The new integral $\mathcal{I}_{2}$ can be expressed in terms of the previously computed wrapping corrections. The explicit expressions in this integral are presented in section D.2.

Next we focus on the term $\log \left(1+Y_{Q}(v \mid \hat{u})\right)$. This term depends both implicitly (through the Bethe root $\hat{u}=u+g^{2} \delta u$ ) and explicitly on $g$. Expanding this gives

$$
\log \left(1+Y_{Q}(v \mid \hat{u})\right)=Y_{Q}^{\mathrm{LO}}(v \mid u)+Y_{Q}^{\mathrm{LO}, 2}(v \mid u)+Y_{Q}^{\mathrm{NLO}}(v \mid u)+\mathcal{O}\left(g^{2 J+8}\right),
$$

where $Y_{Q}^{\mathrm{LO}}(v \mid u)$ is of order $g^{2 J+4}$ and $Y_{Q}^{\mathrm{LO}, 2}(v \mid u), Y_{Q}^{\mathrm{NLO}}(v \mid u)$ are of order $g^{2 J+6}$. Here $Y_{Q}^{\mathrm{NLO}}(v \mid u)$ is obtained by expanding the Bethe root $\hat{u}=u+g^{2} \delta u$ and is expressed as

$$
Y_{Q}^{\mathrm{NLO}}(v \mid u) \equiv g^{2} \partial_{u} Y_{Q}^{\mathrm{LO}}(v \mid u) \delta u .
$$

The resulting contribution to the NLO wrapping correction from this piece is consequently given by

$$
E_{\mathrm{NLO}}^{(2)}(J)=g^{2} \sin p \frac{d}{d u} E_{\mathrm{LO}}^{\mathrm{II}}(J),
$$

where we used (3.3) to express $\delta u=\sin p$.

Subsequently, we turn our attention to $Y_{Q}^{\mathrm{NLO}}(v \mid u)$. It is useful to split this function into two parts

$$
\begin{aligned}
& Y_{r a t}^{\mathrm{NLO}}=2 g^{2} {\left[\frac{4 v^{2}}{\left(Q^{2}+v^{2}\right)^{2}}-\frac{2 v u}{\left(u^{2}+1\right)\left(Q^{2}+v^{2}\right)}-\frac{4}{\left(u^{2}+1\right)\left(Q^{2}+v^{2}\right)}+\frac{J\left(v^{2}-Q^{2}\right)}{\left(Q^{2}+v^{2}\right)^{2}}+\right.} \\
&\left.+\frac{4}{u^{2}+1}-\frac{4\left(Q^{2}-1\right)}{\left(Q^{2}+v^{2}\right)\left(1-Q^{2}-v^{2}+u^{2}\right)}-\frac{1}{Q^{2}+v^{2}}-\frac{4}{\left(u^{2}+1\right)^{2}}\right] Y_{Q}^{0}(v), \\
& Y_{\psi}^{\mathrm{NLO}}=g^{2}\left(\frac{H_{-\frac{Q+i v}{2}}+H_{\frac{i v-Q}{2}}+H_{\frac{Q-i v}{2}}+H_{\frac{Q+i v}{2}}}{u^{2}+1}\right) Y_{Q}^{0}(v) .
\end{aligned}
$$


The contribution $E_{\mathrm{NLO}}^{(3)}$ of the first term $Y_{\text {rat }}^{\mathrm{NLO}}$ can be related, by some partial integrations to integrals that were encountered before. In this respect it is computed in a straightforward manner. The relevant integrals are collected in appendix D. The second term comes from expanding the dressing phase, as presented in appendix A. Note that we here used harmonic numbers $H_{n}$ rather than the digamma function $\psi(n)$. They are related via $H_{n}=\gamma+$ $\psi^{(0)}(n+1)$. Appendix D is devoted to computing the corresponding contribution which we denote as $E_{\mathrm{NLO}}^{(4)}$.

Finally, we consider the term involving the dispersion relation $\mathcal{E}(p)$ from equation (3.1). Since the momentum also receives a wrapping correction, i.e.

$$
p \rightarrow p+g^{2 J+4} \delta p
$$

the asymptotic energy $\mathcal{E}(p)$ also gets corrected

$$
\mathcal{E}(p)=\sqrt{1+4 g^{2} \sin ^{2} \frac{p+\delta p}{2}}=\sqrt{1+4 g^{2} \sin ^{2} \frac{p}{2}}+g^{2 J+6} \sin p \delta p+\mathcal{O}\left(g^{2 J+8}\right) .
$$

The TBA approach relates $\delta p$ to the correction to the asymptotic Bethe equation $\delta \mathcal{R}$ as

$$
\delta p=-\frac{\delta \mathcal{R}}{L_{T B A}} .
$$

Now it is time to recall that for model II the correction $\delta p$ is not proportional to $P_{\mathrm{LO}}^{\mathrm{II}}$. On top of this, from our TBA considerations in section 4 we found that $L_{T B A}=J+1$, which results in the following expression for $\delta p$, cf. formula (6.18),

$$
\delta p=-\frac{J+2}{J+1} P_{\mathrm{LO}}^{\mathrm{II}}-\frac{2}{J+1} \mathcal{I}_{1}(J) .
$$

Before continuing with our discussion of the next-to-leading order correction to the energy, let us mention that there is a consistency check on our result. Each of the above discussed intermediate terms contain polygamma functions. However, just as for the first wrapping correction, we can expect them to cancel in the final result. It turns out that the contribution for polygamma functions indeed exactly cancel out for our rather non-trivial result for $\delta p$.

Collected results. We have explicitly computed the next-to-leading order wrapping corrections for states with $J=2,3,4$ and for one state with $J=6$. We found that in each case the result is a combination of $\zeta$-functions and multiple (double) zeta-functions (see appendix D). We will present the results with all double zeta values expressed in terms of usual $\zeta$-functions. Below we summarize our results.

$\mathbf{J}=\mathbf{2}$. For $J=2$, the Bethe equations admit only the solutions $p=0, \pi$, i.e. $u=\infty$ and $u=0$. The contribution for $p=0$ vanishes, and the NLO wrapping correction for $p=\pi$ is found to be

$$
\frac{E_{\mathrm{NLO}}^{\mathrm{II}}(2)}{g^{10}}=-4 \zeta(3)-\frac{3}{2} \zeta(3)^{2}-5 \zeta(5)+\frac{105}{8} \zeta(7)
$$


$\mathbf{J}=\mathbf{3}$. For $J=3$ the Bethe roots are $u= \pm \frac{1}{\sqrt{3}}$. Both rapidities yield the same wrapping correction

$$
\frac{E_{\mathrm{NLO}}^{\mathrm{II}}(3)}{g^{12}}=\frac{243}{512} \zeta(3)^{2}-\frac{243}{128} \zeta(5)-\frac{405}{512} \zeta(3) \zeta(5)-\frac{21735}{4096} \zeta(7)+\frac{567}{64} \zeta(9) .
$$

$\mathbf{J}=4$. For $J=4$ there are three Bethe roots $u=0, u= \pm 1$. For $u=0$ we find

$$
\begin{aligned}
\frac{E_{\mathrm{NLO}}^{\mathrm{II}}(4)}{g^{14}}=\zeta(3)^{2}-\frac{5}{32} \zeta(5)^{2} & +\zeta(3)\left(\frac{1}{4} \zeta(5)-\frac{7}{4} \zeta(7)\right) \\
& -\frac{9}{2} \zeta(5)-7 \zeta(7)-\frac{21}{4} \zeta(9)+\frac{1155}{64} \zeta(11),
\end{aligned}
$$

while for $u= \pm 1$ the result is

$$
\begin{aligned}
\frac{E_{\mathrm{NLO}}^{\mathrm{II}}(4)}{g^{14}}=-\frac{1}{32} \zeta(3)^{2}- & \frac{5}{256} \zeta(5)^{2}+\zeta(3)\left(\frac{7}{32} \zeta(5)-\frac{7}{32} \zeta(7)\right) \\
& +\frac{9}{64} \zeta(5)-\frac{119}{640} \zeta(7)-\frac{2037}{640} \zeta(9)+\frac{1155}{256} \zeta(11) .
\end{aligned}
$$

$\mathbf{J}=6$. Finally we computed the NLO wrapping correction for $J=6, u=0$ and found

$$
\begin{aligned}
\frac{E_{\mathrm{NLO}}^{\mathrm{II}}(6)}{g^{16}}= & -\frac{5}{2} \zeta(7)-\frac{7}{512} \zeta(7)^{2}-\zeta(5)\left(\frac{3}{32} \zeta(7)+\frac{15}{64} \zeta(9)\right)-\frac{51}{8} \zeta(9)-\frac{957}{128} \zeta(11)+ \\
& +\zeta(3)\left(\frac{3}{4} \zeta(5)+\frac{9}{16} \zeta(7)+\frac{3}{8} \zeta(9)-\frac{99}{64} \zeta(11)\right)-\frac{1287}{256} \zeta(13)+\frac{45045}{2048} \zeta(15)
\end{aligned}
$$

One can see from these explicit results that the term of maximum transcendentality is always of degree $2 J+3$. In case of the leading order wrapping correction this was $2 J+1$.

In the above, we have chosen to express the double zeta-functions in terms of usual $\zeta$ 's and products thereof, but we can also attempt to go the other way around and express the NLO correction purely in terms of double zeta-functions; this turns out to be possible. As a matter of fact, all the wrapping corrections are of the following form

$$
E_{\mathrm{NLO}}^{\mathrm{II}}=g^{2 J+6} \sum_{i>j} A_{i j} \zeta(i, j)
$$

For instance, for $J=2$ this formula explicitly reads

$$
\begin{aligned}
E_{\mathrm{NLO}}^{\mathrm{II}}(2)= & -4 \zeta(2,1)-10(3 \zeta(4,1)+\zeta(3,2))-\frac{3}{2}(16 \zeta(5,1)+9 \zeta(4,2))+ \\
& +\frac{105}{8}(20 \zeta(6,1)+10 \zeta(5,2)+3 \zeta(4,3)) .
\end{aligned}
$$

Since the leading order wrapping correction was of the form

$$
E_{\mathrm{LO}}=g^{2 J+4} \sum_{i} A_{i} \zeta(i),
$$

the general structure that is expected to be found for higher order wrapping corrections will be ordered sums of multiple zeta values. 
We close this section with the following curious observation. As we have seen, the relation between $\delta p$ and $P_{\mathrm{LO}}$ is given by equation (6.34). It is interesting to ask what we would find for the NLO energy correction if we would assume that $\delta p$ and $P_{\mathrm{LO}}$ would be related in the standard fashion: $\delta p=-P_{\mathrm{LO}}$. It turns out that the contribution of $\psi$-functions also cancels out completely in the expression for $E_{\mathrm{NLO}}$ ! The final result differs, however, from $E_{\mathrm{NLO}}^{\mathrm{II}}$, but only in terms linear in $\zeta$-functions. For example, for $J=4, u=1$, the difference is $\frac{21 \zeta(7)}{640}-\frac{21 \zeta(9)}{320}$. In fact, we have found that the following linear combination

$$
P_{\mathrm{LO}}^{\mathrm{II}}+2 \mathcal{I}_{1}(J)
$$

is " $\psi$-free" and, therefore, being added with arbitrary coefficient $\alpha$ to our original expression for $\delta p$ does not spoil the " $\psi$-free" property:

$$
\delta p=-P_{\mathrm{LO}}^{\mathrm{II}}+\frac{\alpha}{J+1}\left(P_{\mathrm{LO}}^{\mathrm{II}}+2 \mathcal{I}_{1}(J)\right) .
$$

When $u=0$ both choices for $\delta p$ give rise to the same NLO correction to the energy. The implications of these observations are not clear to us.

\section{$7 \quad$ Finite-size corrections in $\beta$-deformed and physical orbifold theories}

In this section we apply the generalized Lüscher formulae to compute the leading finite-size corrections to the energies of one- and two-particle excited states from the $\mathfrak{s u}(2)$ and $\mathfrak{s l}(2)$ sectors in $\beta$-deformed theories, and to two-particle states in the $\mathfrak{s l}(2)$ sector of a generic $\mathbb{Z}_{S}$-orbifold. The $Y_{Q}(v)$ functions needed to compute $E_{\mathrm{LO}}$ according to equation (5.1) are constructed by using the twisted transfer matrices and the general formula (2.17). The rest of our considerations follows the same logic as for models I and II. We also make a comparison between our results for $\beta$-deformed and orbifold theories for a single magnon.

\subsection{One-particle $\beta$-deformed states}

For single particle states, we find the following energy corrections for the $\mathfrak{s u}(2)$ and $\mathfrak{s l}(2)$ sectors.

$\mathfrak{s u}(2)$ sector. According to our discussion in section 2, a single magnon in the $\mathfrak{s u}(2)$ sector of $\beta$-deformed theory has the following Bethe equation

$$
1=e^{i(p-2 \pi \beta) J} \quad \Longrightarrow \quad p=2 \pi \beta+\frac{2 \pi n}{J}, \quad n=0, \ldots\left[\frac{J}{2}\right]
$$

For the level-matched case $n=0$ so that a physical magnon has $p=2 \pi \beta$. Its asymptotic energy is

$$
E=J+\sqrt{1+4 g^{2} \sin ^{2} \pi \beta}
$$

On the gauge theory side an operator corresponding to the physical magnon is $\operatorname{tr}\left(\Phi Z^{J}\right)$. 
Let us denote the transfer matrix obtained by twisting the bosonic eigenvalues (in the auxiliary space) with $\alpha$ as $T(v \mid u ; \alpha)$. Implementing the twist describing $\beta$-deformed theory results in the following Y-functions

$$
Y_{Q}(v)=\frac{g^{2 J}}{\left(Q^{2}+v^{2}\right)^{J}} \frac{T_{Q, 1}^{\mathfrak{s u}(2) \ell}(v \mid u ; \beta) T_{Q, 1}^{\mathfrak{s u}(2) r}(v \mid u ;(2 J-1) \beta)}{S_{0}(u, v)},
$$

where the coefficient $S_{0}(u, v)$ is given in appendix A. The leading term in the weak-coupling expansion for the twisted transfer matrix in the $\mathfrak{s u}(2)$ sector is explicitly given by

$$
\begin{aligned}
& T_{Q, 1}^{\mathfrak{s u}(2)}(v \mid u ; \alpha)=\frac{g}{\sqrt{Q^{2}+v^{2}}}\left[\frac{(Q-1)\left((Q+1)^{2}+(v-u)^{2}\right)}{(i(Q-1)+v-u)(i-u)}+\frac{Q e^{i \pi \alpha} \sqrt{\frac{u+i}{u-i}}\left(Q^{2}+(u-v+i)^{2}\right)}{(i(1-Q)-v+u)(i-u)}+\right. \\
& \left.+\frac{Q e^{-i \pi \alpha}(i(Q+1)-v+u)}{(i-u) \sqrt{\frac{u+i}{u-i}}}+\frac{(Q+1)(i(1-Q)+v-u)}{i-u}\right] .
\end{aligned}
$$

This transfer matrix starts at order $g$ which results in the fact that our Y-function, and corresponding corrections to the energy and momentum, will be of order $g^{2 J+2}$.

It is now straightforward to compute the first wrapping correction to the energy. We find it to be a sum of $\zeta$-functions

$$
E_{\mathrm{LO}}^{\beta}(J)=g^{2 J+2} \sum_{n=1}^{\left\lfloor\frac{J+1}{2}\right\rfloor} 16(-1)^{n} \frac{\Gamma(J-n+1) \Gamma\left(J-n+\frac{3}{2}\right)}{\sqrt{\pi} \Gamma(J+1) \Gamma(J-2 n+3)} B_{n}^{\beta}(J) \zeta(2 J-2 n+1),
$$

where the coefficients are given by the following expressions

$$
\begin{aligned}
B_{1}^{\beta}(J) & =-\frac{J}{2} \sin (J \pi \beta) \sin ^{2}(\pi \beta) \sin ((J-2) \pi \beta), \\
B_{n>1}^{\beta}(J) & =\sin (J \pi \beta) \sin ^{2 n}(\pi \beta)[(n-1) \sin ((J-2 n) \pi \beta)-J \cos (\pi \beta) \sin ((J-2 n+1) \pi \beta)] .
\end{aligned}
$$

The leading finite-size correction to the energy found above perfectly agrees with the fieldtheoretic result obtained in [44, 45]. As discussed in the introduction, this is also in agreement with the results of [46].

Special attention has to be paid to the particular case of rational $\beta, \beta=\frac{n}{J}$ with $n=0,1, \ldots, J-1$. For these values of the deformation parameter, the naive lowest order in the weak-coupling expansion of the transfer matrix $T_{Q, 1}^{\mathfrak{s u}(2)}(v \mid u ;(2 J-1) \beta)$ vanishes and the expansion really starts two powers of $g$ higher. Bearing in mind that the rapidity $u$ is $g$-dependent via (3.3) with momentum $p=2 \pi \beta$, it is readily checked that the functions $Y_{Q}$ for these special values of $\beta$ are absolutely the same as for generic $\beta$, up to a shift $J \rightarrow J+1$, so that we find

$$
E_{\mathrm{LO}}^{\beta=n / J}(J)=\left.E_{\mathrm{LO}}^{\beta}(J+1)\right|_{\beta=\frac{n}{J}} .
$$

In particular this implies that for these special values of $\beta$ the expansion of the $Y_{Q}$-functions starts at order $g^{2 J+4}$. 
$\mathfrak{s l}(2)$ sector. In the $\mathfrak{s l}(2)$ sector the single magnon Bethe equations are $e^{i p J}=1$ so that

$$
p=\frac{2 \pi n}{J}, \quad n=0,1, \ldots J-1 .
$$

The physical state must be level-matched leaving only the solution $p=0$. In the $\mathfrak{s l}(2)$ sector of $\beta$-deformed theory the left and right transfer matrices $T_{Q, 1}$ are twisted with $\alpha=0$ and $\alpha=2 J \beta$, respectively. Computing then the corresponding twisted $Y_{Q}$-functions, we find that they all vanish for $p=0$, resulting in a vanishing wrapping correcting to the energy.

\subsection{Two-particle $\beta$-deformed states}

The twist should correctly account for $\beta$-deformation of $\mathcal{N}=4$ SYM for any number of excitations; in this section we will briefly investigate some two-particle states. In particular, we reproduce the wrapping energy correction found in $[45,47]$ for the Konishi state in the $\mathfrak{s u}(2)$ sector of $\beta$-deformed theory, and investigate two-particle states in the $\mathfrak{s l}(2)$ sector for which no field-theoretic results are known so far.

$\mathfrak{s u}(2)$ sector. The perturbative computation of the anomalous dimension of $\beta$-deformed $\mathfrak{s u}(2)$ Konishi-like states [45] agrees perfectly with results coming from the asymptotic Bethe equations up to three loops. At four loops, there is a discrepancy arising due to wrapping effects, which should be accounted for via energy corrections computed from the Lüscher formulae. In [47] a modified S-matrix was used to obtain an expression for the wrapping correction to the energy, which is in agreement with the explicit perturbative results.

The modification of the S-matrix proposed in [47] amounts to giving the matrix elements of the S-matrix for fermionic states a $\beta$-dependent phase:

$$
\mathcal{S}^{(l 1)^{j 1}} \rightarrow \begin{cases}e^{i \pi \beta} \mathcal{S}^{(l 1)^{j 1}}, & j=2 l+1, \ldots, 3 l, \\ e^{-i \pi \beta} \mathcal{S}^{(l 1)^{j 1}}, & j=3 l+1, \ldots, 4 l .\end{cases}
$$

This is obviously different from the twisting procedure we have proposed above, and therefore we cannot a priori expect these two different approaches to agree. However, the $\mathfrak{s u}(2)$ Konishi state is special, because it has particularly symmetric quantum numbers; $J=M$. This means that for such a state our twist amounts to a left twist by $M$ and a right twist by $2 J-M=M$. For such a symmetric twist it is not hard to see that it can effectively be absorbed in the unmodified S-matrices, exactly in the fashion indicated above. This means, in particular, that for the Konishi state the leading wrapping correction computed from the twisted Y-functions must naturally agree with the result of [47]:

$$
E_{\text {Konishi }}^{\beta}=g^{8}\left[-54(1+\Delta)^{3}(-5+3 \Delta) \zeta(3)-360(1+\Delta)^{2} \zeta(5)+\frac{81(1-3 \Delta)^{2}(1+\Delta)^{4}}{(1+3 \Delta)^{2}}\right],
$$

where $\Delta=\frac{\sqrt{5+4 \cos 4 \pi \beta}}{3}$. Of course, we have also verified this agreement by explicit calculation based on the twisted transfer matrix, both analytically for certain nice values of $\beta$, and numerically for various random values of $\beta$.

We would like to emphasize that only in the special case of $J=M$ it is possible to effectively absorb the twist in the modification of the S-matrix in a natural way, and, therefore, these different approaches will only agree in this special case. 
$\mathfrak{s l}(2)$ sector. Now we apply our approach to analyze the wrapping corrections in the $\mathfrak{s l}(2)$ sector of $\beta$-deformed theory. We restrict ourselves to two-particle states with angular momentum $J$, corresponding to the gauge-theory operators schematically of the form $\operatorname{tr}\left(D^{2} Z^{J}\right)$.

To obtain the corresponding $Y_{Q}$-functions, cf. our earlier discussion in section 2, we need to twist the right transfer matrix with a factor of $2 J \pi \beta$ and to keep the left transfer matrix untwisted, regardless of the number of excitations. However, since the Bethe equations in the $\mathfrak{s l}(2)$ sector are not modified under $\beta$-deformation (2.19), the standard level matching condition remains $p=0$. With this twist and the level matching condition $u_{1}=-u_{2}=u$ taken into account, the left transfer matrix for two particles take the following form in the weak coupling limit:

$$
T_{Q, 1}^{\mathfrak{s l}(2) \ell}=\frac{8 g^{2} Q\left(Q^{2}-1+v^{2}-u^{2}\right)}{\left(1+u^{2}\right)\left(Q^{2}+v^{2}\right)\left((Q-1)^{2}-2 i(Q-1) v-v^{2}+u^{2}\right)},
$$

which for $J=2$ is of course nothing else but the transfer matrix for the usual Konishi state. On the other hand, due to the twist, the right transfer matrix starts with a nontrivial zeroth order term

$$
T_{Q, 1}^{\mathfrak{s l}(2) r}=\frac{4 Q \sin ^{2}(\pi J \beta)}{3} \frac{\left(1-Q^{2}+3 v^{2}-3 u^{2}\right)}{(v+i(Q-1))^{2}-u^{2}}
$$

This immediately implies that the wrapping correction will show up at an order $g^{2}$ lower, except for the special values ${ }^{18} \beta=m / J, m=0,1, \ldots, J$. Putting these results together in equation (2.17), yields the $Y_{Q}$-function

$$
Y_{Q}=\sin ^{2}(J \pi \beta) \frac{g^{2 J+2}}{\left(Q^{2}+v^{2}\right)^{2 J+2}} \frac{32 Q^{2}\left(u^{2}+1\right)\left(Q^{2}+v^{2}-u^{2}-1\right)\left(Q^{2}-3 v^{2}+3 u^{2}-1\right)}{3 f_{+}^{+} f_{-}^{+} f_{+}^{-} f_{-}^{-}},
$$

where

$$
f_{\mp}^{ \pm}=(Q \pm 1)^{2}+(v \mp u)^{2} .
$$

Thus, we are now able to compute the wrapping corrections to the energy for two-particle $\mathfrak{s l}(2)$ states with an arbitrary twist. Evaluating the energy integral for arbitrary $u$, gives a string of polygamma functions in addition to the expected $\zeta$-functions. However, substituting the actual solution of the Bethe equations, these polygamma contributions cancel out in a highly non-trivial manner.

Here we explicitly present the wrapping corrections to the energy for a few values of $J$ where the rapidities take particularly simple values; since in the small coupling limit $u_{J, n}=\cot \frac{\pi n}{J+1}$, where $n=1, \ldots,\left\lfloor\frac{J+1}{2}\right\rfloor$, we restrict ourselves to $J=2,3$ and 5 . The results for the corresponding wrapping corrections are summarized in table 3.

\subsection{Physical two-particle $\mathfrak{s l}(2)$ orbifold states}

While the Bethe equations for a two-particle state in the $\mathfrak{s l}(2)$ sector are not modified by the particular orbifolding corresponding to the twist (2.5), the wrapping corrections to the

\footnotetext{
${ }^{18}$ For these values the twist effectively disappears, i.e. we return to the undeformed theory.
} 


\begin{tabular}{|c|c|c|c|}
\hline$J$ & $n$ & $u$ & $E^{\beta}(J) /\left(g^{2 J+2} \sin ^{2}(J \pi \beta)\right)$ \\
\hline 2 & 1 & $1 / \sqrt{3}$ & $\frac{3}{8}$ \\
3 & 1 & 1 & $-\frac{1}{12}-\frac{1}{3} \zeta(3)+\frac{5}{6} \zeta(5)$ \\
3 & 2 & 0 & $-6+\frac{20}{3} \zeta(3)-\frac{5}{3} \zeta(5)$ \\
5 & 1 & $\sqrt{3}$ & $-\frac{1}{1536}-\frac{1}{192} \zeta(3)+\frac{17}{192} \zeta(5)-\frac{161}{384} \zeta(7)+\frac{21}{32} \zeta(9)$ \\
5 & 2 & $1 / \sqrt{3}$ & $-\frac{81}{512}-\frac{27}{64} \zeta(3)+\frac{27}{64} \zeta(5)+\frac{63}{128} \zeta(7)$ \\
5 & 3 & 0 & $-\frac{26}{3}-\frac{4}{3} \zeta(3)+\frac{41}{3} \zeta(5)-\frac{7}{3} \zeta(7)-\frac{21}{16} \zeta(9)$ \\
\hline
\end{tabular}

Table 3. The leading finite-size correction to the energy for certain two-particle states from the $\mathfrak{s l}(2)$ sector in $\beta$-deformed theory with $\beta \neq \frac{m}{J}, m \in \mathbb{Z}$. For $J=2$ the leading correction arises at three loops and for $J=3$ at four. Note that compared to the other two $J=5$ states, the term of maximum transcendentality for $J=5, u=1 / \sqrt{3}$ is missing.

\begin{tabular}{|c|c|c|c|}
\hline$J$ & $S$ & $u$ & $E^{o r b}(J) /\left(g^{2 J} \sin ^{4}(\pi m / S)\right)$ \\
\hline 2 & 1 & $1 / \sqrt{3}$ & $-\frac{1}{3}$ \\
3 & 1 & 1 & $-\frac{1}{18}-\frac{1}{3} \zeta(3)$ \\
3 & 2 & 0 & $-1+\frac{16}{9} \log (2)-\frac{1}{3} \zeta(3)$ \\
5 & 1 & $\sqrt{3}$ & $-\frac{1}{576}-\frac{1}{72} \zeta(3)+\frac{5}{18} \zeta(5)-\frac{35}{36} \zeta(7)$ \\
5 & 2 & $1 / \sqrt{3}$ & $-\frac{3}{64}-\frac{1}{8} \zeta(3)$ \\
5 & 3 & 0 & $-\frac{13}{9}+\frac{16}{9} \zeta(3)-\frac{5}{9} \zeta(5)-\frac{35}{144} \zeta(7)$ \\
\hline
\end{tabular}

Table 4. The leading finite-size correction to the energy for certain two-particle states from the $\mathfrak{s l}(2)$ sector for a $\mathbb{Z}_{S}$-orbifold. The leading order correction enters at order $g^{2 J}$. It is interesting to note that compared to the other two $J=5$ states, the term of maximum transcendentality for $J=5, u=1 / \sqrt{3}$ is missing.

energy are. This is expected because the orbifold only preserves $\mathcal{N}=2$ supersymmetry. To consider two-particle states on a $\mathbb{Z}_{S}$-orbifold, we should give both $\mathfrak{s l}(2)$ transfer matrices the same twist; one of the $S$-th roots of unity. The explicit expression for the lowest order term of the twisted transfer matrix has already been given in equation (7.10), where now all we need to do is replace $2 J \pi \beta$ by $2 \pi m / S$, where $m=0, \ldots, S-1$, and $m=0$ of course gives the usual $\mathfrak{s l}(2)$ descendant of the Konishi state. Using formula (7.10), the leading finite-size correction to the energy of any desired state can be readily computed, here we present the explicit results for some simple states in table 4 . 


\subsection{Comparing $\beta$-deformed and off-shell orbifold magnons}

According to equation (7.1), a single $\mathfrak{s u}(2)$ magnon in $\beta$-deformed theories has momentum $p=2 \pi \beta+\frac{2 \pi n}{J}$. Clearly the case of $\beta$ rational, $\beta=\frac{m}{J}, m \in \mathbb{Z}$, is special, because now $p=\frac{2 \pi(m+n)}{J}$, i.e. the momentum is essentially the same as of an off-shell $\mathfrak{s l}(2)$ magnon in orbifold theory. This suggests the existence of a certain relation between the $\mathfrak{s u}(2)$ magnons of $\beta$-deformed theories with $\beta=\frac{m}{J}$ and $\mathfrak{s l}(2)$ magnons of orbifold theory; their energies must be equal. We could also come to a similar conclusion by inspecting table 1 . Indeed, for $\beta=\frac{m}{J}$ the right twist in the $\mathfrak{s u}(2)$ sector is $(2 J-M) \pi \beta=2 \pi m-\pi \beta M \cong-\pi \beta M$. It is clear now that if for model II the total momentum is chosen to be $p=2 \pi \beta M$, then the twist in the $\mathfrak{s u}(2)$ sector of $\beta$-deformed theory just coincides with the one in model II.

In fact, the equality between the energy of an $\mathfrak{s u}(2)$ magnon in $\beta$-deformed theory with a rational $\beta$ and the energy of the $\mathfrak{s l}(2)$ magnon in the off-shell theory (with $\beta=0$ ) has been already pointed out in [48]. There it was argued to arise due to the symmetry of the spectrum under the shift $\beta \rightarrow \beta+\frac{1}{J}$. In particular, a physical magnon in $\beta$-deformed theory with $\beta=1 / 2$ was shown to have the same leading correction to the energy as a physical magnon with $p=\pi$ in the undeformed theory $[48,66,67]$. The formulae for the energy corrections we have obtained allow us to give a proof of the corresponding statement for arbitrary $J$. Indeed, comparing formula (7.5) taking for $\beta=\frac{n}{J}$ against the wrapping correction (6.2) in model II for $p=\frac{2 \pi n}{J}$ one finds

$$
E^{\beta}(J+1)=E^{\mathrm{II}}(J) .
$$

Actually, the observation made for model II extends ${ }^{19}$ to the level of Y-functions. Namely, we have that

$$
Y_{Q}^{\beta}(J+1)=\frac{\sin ^{2}(\pi(J+1) \beta)}{\sin ^{2}(\pi \beta)} Y_{Q}^{\mathrm{II}}(J)+\frac{\cos (\pi \beta)-\cos (\pi(2 J+1) \beta)}{\sin ^{3}(\pi \beta)} \frac{v-u}{Q^{2}-1+v^{2}-u^{2}} Y_{Q}^{\mathrm{II}}(J) .
$$

We see that for $\beta=\frac{n}{J}$ only the first term on the right hand side of the last expression survives. This also automatically implies that $P_{\mathrm{LO}}^{\beta}$ for $\beta=\frac{n}{J}$ exactly coincides with $P_{\mathrm{LO}}^{\mathrm{II}}$. Notice that the second term in equation (7.13) coincides with the difference between $\delta \mathcal{R}$ and $P_{\mathrm{LO}}^{\mathrm{II}}$.

Finally, we can speculate on the relevance of the NLO result for model II in the context of $\beta$-deformed theories. If for model II the equivalence between the $\mathfrak{s l}(2)$ and the $\mathfrak{s u}(2)$ sectors continues to hold beyond the leading order, it is probable that the results for $E_{\mathrm{NLO}}^{\mathrm{II}}$ we found in section 6 also represent the NLO energy corrections for $\mathfrak{s u}(2)$ states in $\beta$-deformed theory with $\beta=\frac{n}{J}$. Coincidence of $P_{\mathrm{LO}}^{\beta}$ with $P_{\mathrm{LO}}^{\mathrm{II}}$ can be considered as serious evidence in favor of this. However, since currently we do not know what $\delta \mathcal{R}$ is in

\footnotetext{
${ }^{19}$ One can try to compare $\beta$-deformed theory against model I. Here one does not expect any obvious relation, but we still find a certain structural similarity of the leading order corrections to the energy up to normalization and the piece of maximum transcendentality

$$
E^{\beta}(J-1)=-64 \cos ^{4}\left(\frac{m \pi}{2 J}\right) E^{\mathrm{I}}(J)-8 g^{2 J} \sin ^{4}\left(\frac{\pi m}{J}\right) \frac{\Gamma\left(J-\frac{1}{2}\right)}{\sqrt{\pi} \Gamma(J)} \zeta(2 J-3) .
$$


$\beta$-deformed theory and how it is related to $P_{\mathrm{LO}}^{\beta}$, we can not directly verify the equality of the NLO energy corrections.

We could also speculate that in $\beta$-deformed theory the situation might be slightly better, in the sense that possibly $\delta p=P_{\mathrm{LO}}^{\beta}$, in line with our curious observation at the end of section 6 . In that case the corresponding energy correction could be easily obtained from our results for $E_{\mathrm{NLO}}^{\mathrm{II}}$ and it would differ from the later by terms linear in $\zeta$-functions. However, for the moment this is just a bold speculation.

\section{Acknowledgments}

We are grateful to Sergey Frolov for numerous important discussions, and to Zoltan Bajnok and Christoph Sieg for valuable comments on the manuscript. G.A. acknowledges support by the Netherlands Organization for Scientific Research (NWO) under the VICI grant 680-47-602. The work by M.L. and S.T. is a part of the ERC Advanced Grant research programme No. 246974, "Supersymmetry: a window to non-perturbative physics".

\section{A Twisted transfer matrix}

The eigenvalue of the twisted transfer matrix for an anti-symmetric bound state representation with the bound state number $Q$ is given by the following formula, generalizing the result of [61]

$$
\begin{aligned}
& T_{Q, 1}^{\mathfrak{s l}(2)}(v \mid \vec{u})=\prod_{i=1}^{K^{\mathrm{II}}} \frac{y_{i}-x^{-}}{y_{i}-x^{+}} \sqrt{\frac{x^{+}}{x^{-}}}+ \\
& +\prod_{i=1}^{K^{\mathrm{II}}} \frac{y_{i}-x^{-}}{y_{i}-x^{+}} \sqrt{\frac{x^{+}}{x^{-}}}\left[\frac{x^{+}+\frac{1}{x^{+}}-y_{i}-\frac{1}{y_{i}}}{x^{+}+\frac{1}{x^{+}}-y_{i}-\frac{1}{y_{i}}-\frac{2 i Q}{g}}\right] \prod_{i=1}^{K^{\mathrm{I}}}\left[\frac{\left(x^{-}-x_{i}^{-}\right)\left(1-x^{-} x_{i}^{+}\right)}{\left(x^{+}-x_{i}^{-}\right)\left(1-x^{+} x_{i}^{+}\right)} \frac{x^{+}}{x^{-}}\right] \\
& +\sum_{k=1}^{Q-1} \prod_{i=1}^{K^{\mathrm{II}}} \frac{y_{i}-x^{-}}{y_{i}-x^{+}} \sqrt{\frac{x^{+}}{x^{-}}}\left[\frac{x^{+}+\frac{1}{x^{+}}-y_{i}-\frac{1}{y_{i}}}{x^{+}+\frac{1}{x^{+}}-y_{i}-\frac{1}{y_{i}}-\frac{2 i k}{g}}\right]\left\{\prod_{i=1}^{K^{\mathrm{I}}} \lambda_{+}\left(v, u_{i}, k\right)+\prod_{i=1}^{K^{\mathrm{I}}} \lambda_{-}\left(v, u_{i}, k\right)\right\} \\
& -\sum_{k=0}^{Q-1} \prod_{i=1}^{K^{\mathrm{II}}} \frac{y_{i}-x^{-}}{y_{i}-x^{+}} \sqrt{\frac{x^{+}}{x^{-}}}\left[\frac{x^{+}-\frac{1}{x^{+}}-y_{i}-\frac{1}{y_{i}}}{x^{+}-\frac{1}{x^{+}}-y_{i}-\frac{1}{y_{i}}-\frac{2 i k}{g}}\right] \prod_{i=1}^{K^{\mathrm{I}}} \frac{x^{+}-x_{i}^{+}}{x^{+}-x_{i}^{-}} \sqrt{\frac{x_{i}^{-}}{x_{i}^{+}}}\left[1-\frac{\frac{2 i k}{g}}{v-u_{i}+\frac{2}{g}(Q-1)}\right] \times \\
& \times\left\{e^{i \alpha} \prod_{i=1}^{K^{\mathrm{III}}} \frac{w_{i}-x^{+}-\frac{1}{x^{+}}+\frac{i(2 k-1)}{g}}{w_{i}-x^{+}-\frac{1}{x^{+}}+\frac{i(2 k+1)}{g}}+e^{-i \alpha} \prod_{i=1}^{K^{\mathrm{II}}} \frac{y_{i}+\frac{1}{y_{i}}-x^{+}-\frac{1}{x^{+}}+\frac{2 i k}{g}}{y_{i}+\frac{1}{y_{i}}-x^{+}-\frac{1}{x^{+}}+\frac{2 i(k+1)}{g}} \prod_{i=1}^{K^{\mathrm{III}}} \frac{w_{i}-x^{+}-\frac{1}{x^{+}}+\frac{i(2 k+3)}{g}}{w_{i}-x^{+}-\frac{1}{x^{+}}+\frac{i(2 k+1)}{g}}\right\} .
\end{aligned}
$$

Here the twist $e^{i \alpha}$ enters only the last line. Eigenvalues are parametrized by solutions of the auxiliary Bethe equations:

$$
\begin{gathered}
\prod_{i=1}^{K^{\mathrm{I}}} \frac{y_{k}-x_{i}^{-}}{y_{k}-x_{i}^{+}} \sqrt{\frac{x_{i}^{+}}{x_{i}^{-}}}=e^{i \alpha} \prod_{i=1}^{K^{\mathrm{III}}} \frac{w_{i}-y_{k}-\frac{1}{y_{k}}-\frac{i}{g}}{w_{i}-y_{k}-\frac{1}{y_{k}}+\frac{i}{g}}, \\
\prod_{i=1}^{K^{\mathrm{II}}} \frac{w_{k}-y_{i}-\frac{1}{y_{i}}+\frac{i}{g}}{w_{k}-y_{i}-\frac{1}{y_{i}}-\frac{i}{g}}=e^{2 i \alpha} \prod_{i=1, i \neq k}^{K^{\mathrm{III}}} \frac{w_{k}-w_{i}+\frac{2 i}{g}}{w_{k}-w_{i}-\frac{2 i}{g}} .
\end{gathered}
$$


In the formulae above the variable

$$
v=x^{+}+\frac{1}{x^{+}}-\frac{i}{g} Q=x^{-}+\frac{1}{x^{-}}+\frac{i}{g} Q
$$

takes values in the mirror theory rapidity plane, i.e. $x^{ \pm}=x\left(v \pm \frac{i}{g} Q\right)$ where $x(v)$ is the mirror theory $x$-function. As was mentioned above, $u_{j}$ take values in string theory $u$-plane, and therefore $x_{j}^{ \pm}=x_{s}\left(u_{j} \pm \frac{i}{g}\right)$ where $x_{s}(u)$ is the string theory $x$-function. These two functions are given by

$$
x(u)=\frac{1}{2}\left(u-i \sqrt{4-u^{2}}\right), \quad x_{s}(u)=\frac{u}{2}\left(1+\sqrt{1-\frac{4}{u^{2}}}\right) .
$$

Finally, the quantities $\lambda_{ \pm}$are

$$
\begin{array}{r}
\lambda_{ \pm}\left(v, u_{i}, k\right)=\frac{1}{2}\left[1-\frac{\left(x_{i}^{-} x^{+}-1\right)\left(x^{+}-x_{i}^{+}\right)}{\left(x_{i}^{-}-x^{+}\right)\left(x^{+} x_{i}^{+}-1\right)}+\frac{2 i k}{g} \frac{x^{+}\left(x_{i}^{-}+x_{i}^{+}\right)}{\left(x_{i}^{-}-x^{+}\right)\left(x^{+} x_{i}^{+}-1\right)}\right. \\
\left. \pm \frac{i x^{+}\left(x_{i}^{-}-x_{i}^{+}\right)}{\left(x_{i}^{-}-x^{+}\right)\left(x^{+} x_{i}^{+}-1\right)} \sqrt{4-\left(v-\frac{i(2 k-Q)}{g}\right)^{2}}\right] .
\end{array}
$$

For a single string theory particle with rapidity $u$ the twisted $\mathfrak{s l}(2)$ transfer matrix is of the form

$$
T_{Q, 1}^{\mathfrak{s l}(2)}(v \mid u)=1+\frac{\left(x^{-}-x_{1}^{-}\right)\left(1-x_{1}^{+} x^{-}\right)}{\left(x^{+}-x_{1}^{-}\right)\left(1-x_{1}^{+} x^{+}\right)} \frac{x^{+}}{x^{-}}-2 \cos \alpha \frac{\left(x^{+}-x_{1}^{+}\right)}{\left(x^{+}-x_{1}^{-}\right)} \sqrt{\frac{x_{1}^{-}}{x_{1}^{+}}} \frac{Q(u-v)}{(u-v)-\frac{i}{g}(Q-1)}+\lambda,
$$

where

$$
\lambda=(Q-1)\left(1+\frac{\left(x^{+}-x_{1}^{+}\right)\left(x_{1}^{-} x^{+}-1\right)}{\left(x^{+}-x_{1}^{-}\right)\left(x_{1}^{+} x^{+}-1\right)}\right)+\frac{i}{g} Q(Q-1) \frac{x^{+}\left(x_{1}^{-}+x_{1}^{+}\right)}{\left(x_{1}^{-}-x^{+}\right)\left(x_{1}^{+} x^{+}-1\right)}
$$

The matrix $T_{1,1}^{\mathfrak{s l}(2)}(u \mid v)$ is normalized as

$$
T_{1,1}^{\mathfrak{s l}(2)}\left(u_{*} \mid u\right)=1,
$$

which is immediately clear form its expression above. Here the star indicates analytic continuation to the kinematic region of string theory.

The S-matrix in the string-mirror region $S_{\mathfrak{s l}(2)}^{1 * Q}$ is found in [68] (see also [5]) and it has the following weak-coupling expansion

$$
S_{\mathfrak{s l}(2)}^{1 * Q}(u, v)=S_{0}(u, v)+g^{2} S_{2}(u, v)+\ldots,
$$

where

$$
S_{0}(u, v)=-\frac{\left.\left[(v-u)^{2}+(Q+1)^{2}\right][Q-1+i(v-u))\right]}{(u-i)^{2}[Q-1-i(v-u)]} .
$$

and

$$
\begin{aligned}
S_{2}(u, v) & =-S_{0}(v, u) \frac{2\left[2 Q(u-i)+(u+i)\left(v^{2}+Q^{2}+2 v(u-i)\right)\right]}{\left(v^{2}+Q^{2}\right)\left(1+u^{2}\right)}+ \\
& \frac{S_{0}(v, u)}{1+u^{2}}\left[4 \gamma+\psi\left(1+\frac{Q+i v}{2}\right)+\psi\left(1-\frac{Q+i v}{2}\right)+\psi\left(1+\frac{Q-i v}{2}\right)+\psi\left(1-\frac{Q-i v}{2}\right)\right] .
\end{aligned}
$$

These expressions are enough to build up the two leading terms in the weak-coupling expansion of the asymptotic function $Y_{Q}^{o}$. 


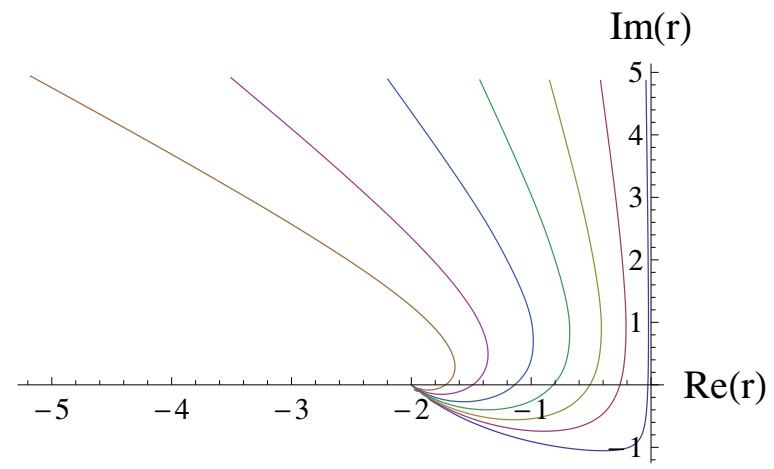

Figure 7. The behaviour of zeroes of $Y_{+}-1$ as $g$ increases, for $p$ taking the values $\frac{9999 \pi}{10000}, \frac{3 \pi}{4}, \frac{\pi}{2}$, $\frac{\pi}{3}, \frac{\pi}{5}, \frac{\pi}{10}, \frac{\pi}{100}$ from left to right. For negative values of momenta the picture should naturally be mirrored with respect to the imaginary axis.

\section{B Asymptotic critical points and TBA}

As mentioned in the main text, a single magnon shows a critical behaviour quite similar to what has been observed in the two-particle case in [17]. However, we would like to stress that the discussion of criticality is restricted to the asymptotic solution; the data recently obtained for the two-particle case [24] is not sufficient to conclusively establish the existence of critical points for an exact solution.

For model I we find critical behaviour that arises from the movement of zeroes of $Y_{+}-1$ and poles of $Y_{M \mid w}$ and $Y_{M \mid v w}$ in the complex plane. These zeroes and poles run into the integration contour at certain values of $g$ and further cross it, yielding extra contributions in the TBA equations above these what are called critical values of $g$.

We indicated before that zeroes of $1-Y_{+}$and poles of $Y_{M \mid w}$ and $Y_{M \mid v w}$ are related by shifts of $i / g$; owing to this we can treat their critical behaviour in a unified manner. For concreteness however, let us first consider zeroes of $Y_{+}-1$. The function $Y_{+}$has the interval $(-2,2)$ as its associated canonical integration contour, and as is plotted in figure 7 the zeroes of $Y_{+}-1$ cross this contour, and do so at a particular value of $g$ denoted $g_{c r}^{0}$. As these zeroes do so from either side of the real line they drag the integration contour along with them. In order to maintain the canonical integration contour in the TBA equations, one needs to pick up a contribution from each of these zeroes as they move back into the complex plane, as illustrated in figure 8. For the poles of $Y_{M \mid w}$ and $Y_{M \mid v w}$ the story is nearly identical as they show exactly the same behaviour, except that the crossing happens at higher values in $g$. The critical values as a function of the particle momentum $p$ have been plotted for the first few $M$ in figure 9. The contributions these zeroes give in the TBA above the critical values can straightforwardly be read of from the ground state TBA equations taking into account the orientation of the integration contour.

In model II the story is different as $1-Y_{+}$has no zeroes, but now the zeroes $r_{M}$ show criticality. As discussed in the main text, we have found exact expressions for the asymptotic zeroes, allowing us to understand and treat the asymptotic criticality exactly. Concisely the story is as follows; a zero $r_{M}$ can be imaginary but between the real line 


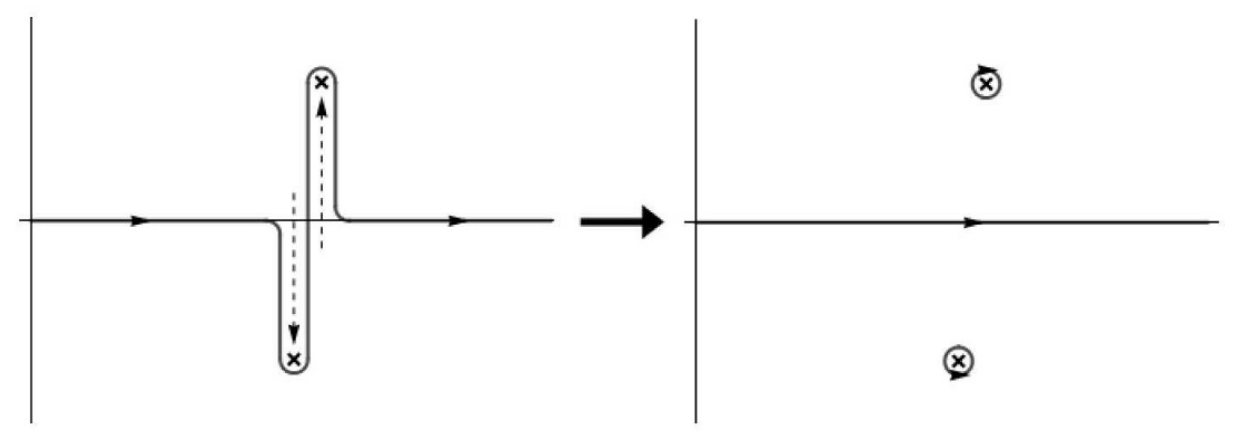

Figure 8. The zeroes of $Y_{+}$crossing the real line giving extra contributions as the integration contour is returned to the real line.

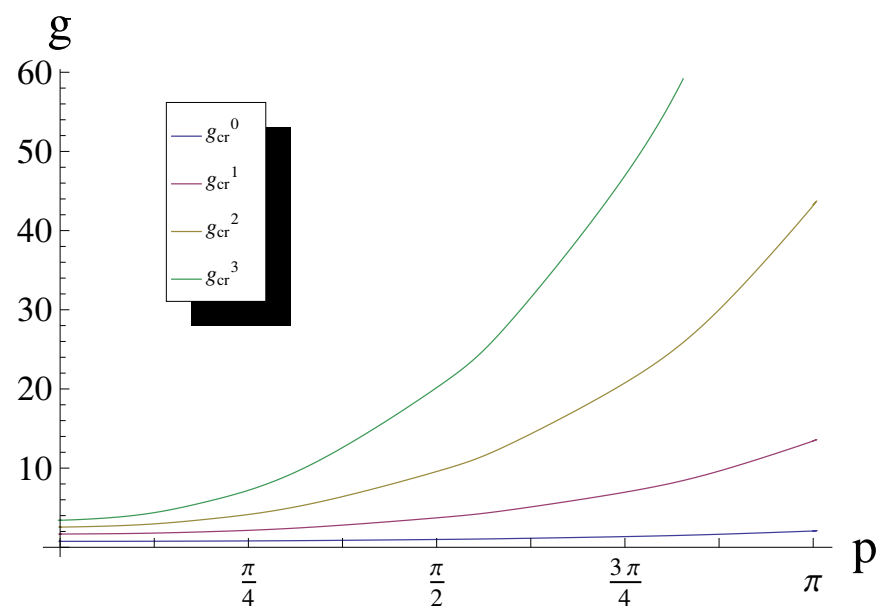

Figure 9. The critical values $g_{c r}^{M}$ as $p$ varies from 0 to $\pi$.

and $-i / g$ at small coupling for a given state (momentum). When this is the case, the zero should be accounted in the excited TBA equations, but as $g$ is increased the zero moves away from the real line and crosses either the real line or $-i / g$. The value of $g$ for which this happens can also be called a critical value, where now beyond the critical value a zero $r_{M}$ no longer contributes to the TBA equations. This story can be readily verified by making use of the explicit expression (4.5). Please note that in this twisted model, for a carefully chosen state these critical values can be arbitrarily small.

\section{Wrapping corrections}

In this section we present the LO corrections to the energy and momentum in full generality for both model I and II. 


\section{C.1 Model I}

We write $\psi^{(m)}$ for the $m$-th polygamma function, $\psi^{(m)}=\frac{d^{m} \psi^{(0)}(z)}{d z^{m}}$, where $\psi \equiv \psi^{0}(z)=$ $\Gamma^{\prime}(z) / \Gamma(z)$. Let us first define

$$
E_{\mathrm{LO}}^{\mathrm{I}}=\frac{\left(u-\sqrt{u^{2}+1}\right)^{2}}{2 \pi} \mathscr{E} .
$$

Then, we introduce the following quantities

$$
\begin{aligned}
A(J) & =J\left[\psi^{(J-1)}\left(\frac{1-i u}{2}\right)-(-1)^{J} \psi^{(J-1)}\left(\frac{1+i u}{2}\right)\right], \\
B_{m}(J) & =\frac{\Gamma(J-m) \Gamma\left(J-m+\frac{1}{2}\right)}{\Gamma(J-2(m-1))}\left[\frac{m-1+i(J-m) u}{(1+i u)^{2 m}}+\frac{m-1-i(J-m) u}{(1-i u)^{2 m}}\right] .
\end{aligned}
$$

The full leading order wrapping correction to the energy for $J>4$ is given by

$$
\begin{aligned}
\frac{\mathscr{E}(J)}{g^{2 J}}= & \frac{\pi\left[(i u+1)^{J}-(i u-1)^{J}\right]}{2^{J}(J-1) !\left(u^{2}+1\right)^{J-1}}\left\{\frac{A(J)}{4 J}+\frac{i u}{u^{2}+1} A(J-1)-\frac{J-1}{u^{2}+1} A(J-2)\right\}+ \\
& -\frac{\sqrt{\pi}}{(J-1) !} \sum_{m=1}^{\left\lfloor\frac{J}{2}\right\rfloor+1} B_{m}(J) \zeta(2 J-2 m-1) .
\end{aligned}
$$

Formula (C.4) was checked analytically up to $J=8$ and numerically up to $J=200$. It is also readily seen that the energy correction is real. For $J=3,4$ formula (C.4) is not well defined due to the divergence $\zeta(1)=\infty$. This can be resolved by taking the appropriate values of $J, n$ and substituting $\zeta(1) \rightarrow \gamma_{E}$ in (C.4). After this substitution, formula (C.4) holds. Explicitly, for $J=3$ one gets

$$
\begin{aligned}
\frac{\mathscr{E}(3)}{g^{6}}= & \frac{\pi\left[(i u+1)^{3}-(i u-1)^{3}\right]}{16\left(u^{2}+1\right)^{2}}\left\{\frac{A(3)}{12}+\frac{i u}{u^{2}+1} A(2)-\frac{2}{u^{2}+1} A(1)\right\}+ \\
& +\frac{\pi}{2} \frac{\left(1-3 u^{2}\right) \gamma_{E}+3 u^{2}\left(1+u^{2}\right) \zeta(3)}{\left(u^{2}+1\right)^{3}} .
\end{aligned}
$$

For $J=2$ one finds $(A(0)=0)$

$$
\frac{\mathscr{E}(2)}{g^{4}}=\frac{\pi\left[(i u+1)^{2}-(i u-1)^{2}\right]}{4\left(u^{2}+1\right)}\left\{\frac{A(2)}{8}+\frac{i u}{u^{2}+1} A(1)\right\}+\frac{\pi}{2} \frac{\left(1-4 \gamma_{E}\right) u^{2}-1}{\left(u^{2}+1\right)^{2}} .
$$

Finally, for $J=1$ the sum over $M$ does not converge and we find that the wrapping correction diverges. Nevertheless, expression (C.4) can be continued to $J=1$ and it gives a finite result

$$
\frac{\mathscr{E}(1)}{g^{2}}=-\frac{\pi}{12}\left[\frac{4 u^{2}}{\left(u^{2}+1\right)^{2}}+3 \psi\left(\frac{1-i u}{2}\right)+3 \psi\left(\frac{1+i u}{2}\right)\right] .
$$

So far the formulae for the energy correction were obtained for arbitrary values of particle rapidity $u$, i.e. not necessarily satisfying the quantization condition. If we impose the quantization condition (the Bethe equations), then at leading order in $g$ the rescaled rapidity is $u=\cot \frac{\pi n}{J}$ with $n$ integer. For these values of $u$ the following relation holds

$$
(i u+1)^{J}-(i u-1)^{J}=\frac{2^{J}}{\left(1-e^{\frac{2 \pi i n}{J}}\right)^{J}}\left(1-e^{2 \pi i n}\right)=0 .
$$

Therefore, the contribution of the polygamma-functions to the energy correction completely drops out on solutions of the Bethe equation, resulting in formula (5.3). 


\section{C.2 Model II}

Energy. To present the value of the energy integral (5.1) for model II, we define the following quantities

$$
\begin{aligned}
& A(J)=\left[\psi^{(J)}\left(\frac{1-i u}{2}\right)+(-1)^{J} \psi^{(J)}\left(\frac{1+i u}{2}\right)\right], \\
& B_{m}(J)=\frac{\Gamma(J-m+1) \Gamma\left(J-m+\frac{3}{2}\right)}{\Gamma(J-2(m-1))}\left[\frac{m+i(J-m+1) u}{(1+i u)^{2 m}}+\frac{m-i(J-m+1) u}{(1-i u)^{2 m}}\right] .
\end{aligned}
$$

Then we find for $J>2$

$$
\begin{aligned}
\frac{E_{\mathrm{LO}}^{\mathrm{II}}(J)}{g^{2 J+4}}= & \frac{(i u-1)^{J}-(i u+1)^{J}}{2^{J} J !\left(u^{2}+1\right)^{J+1}}\left\{\frac{A(J)}{2(J+1)}+\frac{2 i u}{u^{2}+1} A(J)-\frac{2 J}{u^{2}+1} A(J-1)\right\}+\quad \text { (C.11) } \\
& +\frac{8}{\left(1+u^{2}\right)^{2} \sqrt{\pi}(J+1) !} \sum_{m=1}^{\left\lfloor\frac{J}{2}\right\rfloor+1} B_{m}(J) \zeta(2 J-2 m+1)-\frac{8}{\left(1+u^{2}\right)^{2}} \frac{\Gamma\left(J+\frac{3}{2}\right)}{\Gamma(J+2)} \frac{\zeta(2 J+1)}{\sqrt{\pi}} .
\end{aligned}
$$

Formula (C.11) was checked analytically up to $J=8$ and numerically up to $J=300$. For $J=2$ formula (C.4) is not well defined, again because of the divergence $\zeta(1)=\infty$. However, for $J=2$, the energy correction can be easily evaluated directly

$$
\frac{E_{\mathrm{LO}}^{\mathrm{II}}(2)}{g^{8}}=\frac{(i u-1)^{2}-(i u+1)^{2}}{8\left(u^{2}+1\right)^{3}}\left\{\frac{A(3)}{6}+\frac{2 i u A(2)}{u^{2}+1}-\frac{4 A(1)}{u^{2}+1}\right\}+\frac{\left(6 u^{2}+2\right) \zeta(3)}{\left(u^{2}+1\right)^{4}}-\frac{5 \zeta(5)}{2\left(u^{2}+1\right)^{2}} .
$$

Finally, for $J=1$ the sum over $M$ does not converge and, therefore, the leading wrapping correction diverges.

Momentum. Define

$$
\begin{aligned}
D_{m}(J)= & i \frac{2^{2 m-2 J+2} \Gamma(2 J-2 m+2)}{\Gamma(J+3) \Gamma(J-2 m+2)}\left[\frac{(J-m+1)(2 m+1)}{1+u^{2}}\left(\frac{1}{(1+i u)^{2 m+2}}-\frac{1}{(1-i u)^{2 m+2}}\right)+\right. \\
& \left.+\frac{2(J+2) m}{\left(1+u^{2}\right)^{2}}\left(\frac{1}{(1+i u)^{2 m+1}}-\frac{1}{(1-i u)^{2 m+1}}\right)\right]
\end{aligned}
$$

then

$$
\begin{aligned}
P_{\mathrm{LO}}^{\mathrm{II}}= & -\sum_{m=0}^{\left\lfloor\frac{J+1}{2}\right\rfloor} D_{m}(J) \zeta(2 J-2 m+1)+ \\
& +i \frac{(i u-1)^{J}-(i u+1)^{J}}{2^{J+2}(J+2) !\left(u^{2}+1\right)^{J+1}} A(J+2)+i \frac{(i u+1)(i u-1)^{J}-(i u-1)(i u+1)^{J}}{2^{J+1}(J+1) !\left(u^{2}+1\right)^{J+2}} A(J+1) \\
& -i \frac{(i u-1)^{J+2}-(i u+1)^{J+2}}{2^{J} J !\left(u^{2}+1\right)^{J+3}} A(J)-i \frac{(i u+1)(i u-1)^{J+2}-(i u-1)(i u+1)^{J+2}}{2^{J-1}(J-1) !\left(u^{2}+1\right)^{J+4}} A(J-1) .
\end{aligned}
$$

On the solution of the Bethe equations the polygamma functions do not drop out.

\section{Details on the NLO computation}

In this appendix we have gathered some details on the explicit computation of the NLO correction to the energy. 


\section{D.1 The Harmonic number integral}

Special attention has to be paid to the contribution $Y_{\psi}^{\mathrm{NLO}}$ due to the appearance of harmonic numbers. Let us first set up the tools to handle sums and integrals of these numbers. The definition of the multiple (double in this case) zeta value $\zeta(a, b)$ is given by

$$
\zeta(a, b) \equiv \sum_{n>m} \frac{1}{n^{a}} \frac{1}{m^{b}} .
$$

For integer values of $a, b$ this can generically be written in terms of regular $\zeta$ values and products thereof. A useful program on the web for doing this is EZFace. ${ }^{20}$ These multiple zeta values can then be related to sums of polygamma function via harmonic sums [69]

$$
\sum_{Q=1}^{\infty} \frac{\psi^{(b)}(Q)}{Q^{a}}=(-1)^{b+1} b ![\zeta(a) \zeta(b+1)-\zeta(a, b+1)] .
$$

We will also encounter more generic sums including $\psi$-functions. Following [5] we can write for sums of polygamma's

$$
\sum_{Q=1}^{\infty} \Sigma(Q) \psi^{(b)}(Q)=(-1)^{b} \int_{0}^{\infty} d t \mathcal{L}^{-1}(\Sigma) \Gamma(b+1) \frac{\operatorname{Li}_{b+1}\left(e^{-t}\right)-\zeta(b+1)}{e^{t}-1},
$$

where $\mathcal{L}^{-1}(\Sigma)$ is the inverse Laplace transform of $\Sigma(Q)$. For the digamma function one finds

$$
\sum_{Q=1}^{\infty} \Sigma(Q) \psi^{(0)}(Q)=\int_{0}^{\infty} d t \mathcal{L}^{-1}(\Sigma) \frac{\log \left(1-e^{-t}\right)+\gamma}{1-e^{t}} .
$$

In general when one encounters rational functions $\sum_{Q=1}^{\infty} \Sigma(Q) \psi^{(b)}(Q)$, the recipe that proved useful in explicit computations was to split it into two parts $\Sigma(Q)=\Sigma_{0}(Q)+\Sigma_{1}(Q)$, where

$$
\Sigma_{0}(Q)=\sum_{n>1} \frac{a_{n}}{Q^{n}}
$$

To the term containing $\Sigma_{0}$ one can then apply formula (D.2) and on the remainder $\Sigma_{1}$ one can use the integral relations (D.3) and (D.4).

Let us now apply the above discussion to the integral $E_{\mathrm{NLO}}^{(4)}$. The integral is most easily evaluated by summing residues. The integrand has three different types of poles in the upper half-plane

- dynamical poles at $u+i(Q \pm 1)$, giving a contribution $E_{\mathrm{NLO}, d y n}^{(4)}$

- poles at $i(Q+2 k)$ for $k \in \mathbb{Z}_{>0}$, giving $E_{\mathrm{NLO}, k}^{(4)}$

- pole at $i Q$, resulting in $E_{\mathrm{NLO}, i Q}^{(4)}$

All of the above poles give rise to residues that contribute to the integral and we will discuss them separately.

\footnotetext{
${ }^{20}$ http://oldweb.cecm.sfu.ca/projects/EZFace/.
} 
Dynamical poles. Let us first consider the residue at the points $v=u+i(Q \pm 1)$. One straightforwardly finds

$$
\begin{aligned}
& \operatorname{res}_{v=u+i(Q+1)} Y_{\psi}^{\mathrm{NLO}}=\frac{8 i Q(Q+1)\left(H_{Q-\frac{i u-1}{2}}+H_{\frac{i u-1}{2}-Q}+H_{\frac{1-i u}{2}}+H_{\frac{(i u-1)}{2}}\right)}{(u+i)^{J}\left(u^{2}+1\right)^{3}(2 i Q+u+i)^{2}(2 i Q+u+i)^{J}}, \\
& \operatorname{res}_{v=u+i(Q-1)} Y_{\psi}^{\mathrm{NLO}}=\frac{8 i Q(Q-1)\left(H_{Q-\frac{i u+1}{2}}+H_{\frac{i u+1}{2}-Q}+H_{-\frac{i u+1}{2}}+H_{\frac{i u+1}{2}}\right)}{(u-i)^{J}\left(u^{2}+1\right)^{3}(-2 Q+i u+1)^{2}(2 i Q+u-i)^{J}} .
\end{aligned}
$$

Both contributions have to be added and then summed over $Q$. The obtained expression naturally splits in two pieces, one which is purely rational and one that also contains terms of the form $\psi\left(Q+\frac{1+i w}{2}\right)$. The latter can be summed by making use of the techniques of [5] used to derive equations (D.2)-(D.4). This yields the following result for the first piece

$$
E_{\mathrm{NLO}, d y n}^{(4), 1}=\frac{i(-1)^{J}(u+i)^{J}}{\left(u^{2}+1\right)^{J+3} 2^{J-1}}\left[\frac{\psi^{(J)}\left(\frac{1-i u}{2}\right)}{J !}-\frac{\left(u^{2}+1\right)}{4} \frac{\psi^{(J+2)}\left(\frac{1-i u}{2}\right)}{(J+2) !}-i u \frac{\psi^{(J+1)}\left(\frac{1-i u}{2}\right)}{(J+1) !}\right] \text {. }
$$

The remaining rational part can be more straightforwardly summed. Adding up both contributions gives

$$
E_{\mathrm{NLO}, d y n}^{(4)}=\frac{-8 i^{J} u}{2^{J}\left(u^{2}+1\right)^{4}(u-i)^{J}}\left[\frac{u^{2}+1}{4} \frac{\psi^{(J+1)}\left(\frac{1-i u}{2}\right)}{(J+1) !}+i u \frac{\psi^{(J)}\left(\frac{1-i u}{2}\right)}{J !}-\frac{\psi^{(J-1)}\left(\frac{1-i u}{2}\right)}{(J-1) !}\right] .
$$

In deriving the above results we used the fact that the Bethe root $u$ satisfies the Bethe equations $\left(\frac{u-i}{u+i}\right)^{J}=1$. As it is an intermediate result, the quantity $E_{\mathrm{NLO}, d y n}^{(4)}$ is not real by itself. It will be canceled in the final energy correction as it should because it solely consists of $\psi$-functions.

Poles at $\boldsymbol{i}(\boldsymbol{Q}+\mathbf{2 k})$. The integrand also exhibits a pole at $i(Q+2 k)$ due to the harmonic numbers. The residue at this point is easily found to be

$$
\operatorname{res}_{i(Q+2 k)} Y_{\psi}^{\mathrm{NLO}}=4 i Y_{Q}^{\mathrm{LO}}(i(Q+2 k), u) .
$$

In other words, this gives a total contribution of

$$
E_{\mathrm{NLO}, k}^{(4)}=4 \sum_{k=1}^{\infty} \sum_{Q=1}^{\infty} Y_{Q}^{\mathrm{LO}}(i(Q+2 k), u) .
$$

It is most convenient to first preform the sum over $Q$. This yields, again on a solution of the Bethe equation,

$$
\begin{aligned}
& \sum_{Q=1}^{\infty} Y_{Q}^{\mathrm{LO}}(i(Q+2 k), u)= \\
& \frac{2^{1-J}(-i)^{J}}{(u+i)^{J}\left(u^{2}+1\right)^{3}} \frac{u+i-2 i k}{k^{J+2}}+\sum_{n=0}^{J-2} B_{n}^{\psi}(J)-\frac{2^{3-2 J} i}{\left(u^{2}+1\right)^{3}} \frac{\psi^{(J)}(k+1)}{k^{J+1} J !}+ \\
& -\frac{2^{4-2 J}}{\left(u^{2}+1\right)^{3}} \frac{u}{k^{J}\left[(2 k+i u)^{2}-1\right]}\left[\frac{\left(u^{2}+1\right) \psi^{(J+1)}(k+1)}{4 i u(J+1) !}+\frac{\psi^{(J)}(k+1)}{J !}-\frac{\psi^{(J-1)}(k+1)}{i u(J-1) !}\right]
\end{aligned}
$$


where

$$
B_{n}^{\psi}(J)=\frac{(-i)^{n}}{2^{2 J-n-1}}\left[\frac{1}{(u+i)^{n+1}}-\frac{1}{(u-i)^{n+1}}\right]\left[\frac{u^{2}+1}{k^{J+2}}-\frac{4 i u}{k^{J+1}}-\frac{4}{k^{J}}\right] \frac{\psi^{(J-n-1)}(k+1)}{\left(u^{2}+1\right)^{3} \Gamma(J-n)} .
$$

By using the relation

$$
\psi^{(n)}(k+1)=\psi^{(n)}(k)+\frac{(-1)^{n} n !}{k^{n+1}},
$$

it is now straightforward to sum first line over $k$ by means of the previously discussed techniques.

The terms from the second line are all proportional to $\frac{u}{k^{J}\left[(2 k+i u)^{2}-1\right]}$. This function can easily be split up in the following way

$$
\frac{1}{k^{J}\left[(2 k+i u)^{2}-1\right]}=\frac{(2 i)^{J}}{(u+i)^{J}} \frac{1}{(2 k+i u)^{2}-1}+\sum_{n=0}^{J-2}\left[\frac{1}{(u+i)^{n}}-\frac{1}{(u-i)^{n}}\right] \frac{(2 i)^{n-2}}{k^{J-n}}
$$

The tail allows for a direct expression in terms of multiple zeta values. The first piece has to be inverse Laplace transformed

$$
\mathcal{L}^{-1}\left(\frac{1}{(2 k+i u)^{2}-1}\right)=\frac{1}{4}\left(e^{t}-1\right) e^{-\frac{1}{2} t(1+i u)}
$$

One can then explicitly preform the integral (D.3) to find

$$
\begin{aligned}
& \int d t \mathcal{L}^{-1}\left(\frac{1}{(2 k+i u)^{2}-1}\right) \Gamma(k+1) \frac{\operatorname{Li}_{k+1}\left(e^{-t}\right)-\zeta(k+1)}{e^{t}-1}= \\
& \quad \frac{2 i(-1)^{k} k ! \zeta(k+1)}{u-i}-(-1)^{k}(2 i)^{k+1} k ! \frac{H_{\frac{1}{2} i(u+i)}}{(u+i)^{k+1}}-(-1)^{k} k ! \sum_{n=1}^{k} \frac{(2 i)^{n} \zeta(k-n+2)}{(u+i)^{n}} .
\end{aligned}
$$

Pole at $i Q$. The residue at $v=i Q$ is the most complicated one. It receives contributions from both the rational part and the harmonic number part. Let us expand the harmonic number part around this point

$$
\begin{aligned}
& H_{-\frac{Q+i v}{2}}+H_{\frac{i v-Q}{2}}+H_{\frac{Q-i v}{2}}+H_{\frac{Q+i v}{2}}= \\
& \frac{2 i}{v-i Q}+\left(H_{Q}+H_{Q-1}\right)-\sum_{n=2}\left(\frac{1}{2 i}\right)^{n} \frac{2(-1)^{n+1} \psi^{(n)}(1)-\psi^{(n)}(Q)-\psi^{(n)}(Q+1)}{n !}(v-i Q)^{n} .
\end{aligned}
$$

For any value of $J$ one can now easily extract the residue at this point and apply the discussed techniques. 


\section{D.2 Some integrals}

In this section we present a list with integrals that are encountered throughout the main text. These integrals are

$$
\begin{aligned}
& \mathcal{I}_{1}=\frac{1}{2 \pi} \sum_{Q=1}^{\infty} \int d v \frac{v-u}{Q^{2}-1+v^{2}-u^{2}} Y_{Q}^{o}(v), \\
& \mathcal{I}_{2}=\frac{\left(u^{2}+1\right)^{2}}{2 \pi} \sum_{Q=1}^{\infty} \int d v \frac{4 v^{2}}{\left(Q^{2}+v^{2}\right)^{2}} Y_{Q}^{o}(v), \\
& \mathcal{I}_{3}=\frac{1}{2 \pi} \sum_{Q=1}^{\infty} \int d v \frac{\left(Q^{2}-1\right)}{\left(Q^{2}+v^{2}\right)\left(1-Q^{2}-v^{2}+u^{2}\right)} Y_{Q}^{o}(v) .
\end{aligned}
$$

Integral $\mathcal{I}_{1}$ is computed similarly to $E_{\mathrm{LO}}$. One finds

$$
2 \pi \mathcal{I}_{1}(J)=\frac{A_{\mathcal{I}}(J+1)+B_{\mathcal{I}}(J+1)}{\left(u^{2}+1\right)^{2}(J+1) !}-\frac{A_{\mathcal{I}}(J+2)+B_{\mathcal{I}}(J+2)}{\left(u^{2}+1\right)(J+2) !},
$$

where the coefficients in the above expression are

$$
\begin{aligned}
\frac{A_{\mathcal{I}}(J)}{2 \pi i}= & \frac{J}{2^{J}}\left[\frac{1}{(u+i)^{J-1}}-\frac{1}{(u-i)^{J-1}}\right]\left[\psi^{(J-1)}\left(\frac{1-i u}{2}\right)-(-1)^{J} \psi^{(J-1)}\left(\frac{1+i u}{2}\right)\right]+ \\
& +\frac{J(J-1)}{2^{J-1}}\left[\frac{1}{(u+i)^{J}}-\frac{1}{(u-i)^{J}}\right]\left[\psi^{(J-2)}\left(\frac{1-i u}{2}\right)+(-1)^{J} \psi^{(J-2)}\left(\frac{1+i u}{2}\right)\right], \\
\frac{B_{\mathcal{I}}(J)}{2 \pi i}= & \left.\frac{2 J}{\sqrt{\pi}} \sum_{n=1}^{\left\lfloor\frac{J}{2}\right\rfloor} \frac{\Gamma(J-n) \Gamma\left(J-n+\frac{1}{2}\right)}{\Gamma(J-2 n+1)}\left[\frac{1}{(u-i)^{2 n}}-\frac{1}{(u+i)^{2 n}}\right] \zeta(2 J-2 n-1) . \quad \text { (D. } 24\right)
\end{aligned}
$$

One can then express the remaining integrals $\mathcal{I}_{2,3}$ in terms of the previously computed integrals and derivatives thereof

$$
\begin{aligned}
\mathcal{I}_{2}(J)=- & \frac{32 E_{\mathrm{LO}}^{\mathrm{I}}(J+2)}{(J+2)(J+3)}-\frac{d}{d u} \frac{\left(u^{2}+1\right)^{2} P_{\mathrm{LO}}^{\mathrm{II}}(J)}{J+3}+ \\
& +\frac{d}{d u} \frac{4\left(u^{2}+1\right)^{2} \mathcal{I}_{1}(J)}{(J+2)(J+3)}+\frac{2\left(u^{2}+1\right)^{2} E_{\mathrm{LO}}^{\mathrm{II}}(J+1)}{J+3}, \\
\mathcal{I}_{3}(J)= & u \mathcal{I}_{1}(J+1)-E_{\mathrm{LO}}^{\mathrm{II}}(J+1)-\frac{4 E_{\mathrm{LO}}^{\mathrm{I}}(J+2)+2 \partial_{u}\left[\mathcal{I}_{1}(J)\left(u^{2}+1\right)\right]}{(J+2)\left(u^{2}+1\right)^{2}} .
\end{aligned}
$$

Open Access. This article is distributed under the terms of the Creative Commons Attribution Noncommercial License which permits any noncommercial use, distribution, and reproduction in any medium, provided the original author(s) and source are credited.

\section{References}

[1] J.M. Maldacena, The large- $N$ limit of superconformal field theories and supergravity, Int. J. Theor. Phys. 38 (1999) 1113 [Adv. Theor. Math. Phys. 2 (1998) 231] [hep-th/9711200] [SPIRES]. 
[2] M. Lüscher, Volume Dependence of the Energy Spectrum in Massive Quantum Field Theories. 1. Stable Particle States, Commun. Math. Phys. 104 (1986) 177 [SPIRES].

[3] R.A. Janik and T. Lukowski, Wrapping interactions at strong coupling - the giant magnon, Phys. Rev. D 76 (2007) 126008 [arXiv:0708.2208] [SPIRES].

[4] Z. Bajnok and R.A. Janik, Four-loop perturbative Konishi from strings and finite size effects for multiparticle states, Nucl. Phys. B 807 (2009) 625 [arXiv:0807.0399] [SPIRES].

[5] Z. Bajnok, A. Hegedus, R.A. Janik and T. Lukowski, Five loop Konishi from AdS/CFT, Nucl. Phys. B 827 (2010) 426 [arXiv:0906.4062] [SPIRES].

[6] A.V. Kotikov, L.N. Lipatov, A. Rej, M. Staudacher and V.N. Velizhanin, Dressing and Wrapping, J. Stat. Mech. (2007) P10003 [arXiv:0704.3586] [SPIRES].

[7] Z. Bajnok, R.A. Janik and T. Lukowski, Four loop twist two, BFKL, wrapping and strings, Nucl. Phys. B 816 (2009) 376 [arXiv:0811.4448] [SPIRES].

[8] T. Lukowski, A. Rej and V.N. Velizhanin, Five-Loop Anomalous Dimension of Twist-Two Operators, Nucl. Phys. B 831 (2010) 105 [arXiv:0912.1624] [SPIRES].

[9] M. Beccaria, V. Forini, T. Lukowski and S. Zieme, Twist-three at five loops, Bethe Ansatz and wrapping, JHEP 03 (2009) 129 [arXiv:0901.4864] [SPIRES].

[10] F. Fiamberti, A. Santambrogio, C. Sieg and D. Zanon, Wrapping at four loops in $N=4$ SYM, Phys. Lett. B 666 (2008) 100 [arXiv:0712.3522] [SPIRES].

[11] V.N. Velizhanin, The four-loop anomalous dimension of the Konishi operator in $N=4$ supersymmetric Yang-Mills theory, JETP Lett. 89 (2009) 6 [arXiv:0808.3832] [SPIRES].

[12] F. Fiamberti, A. Santambrogio and C. Sieg, Five-loop anomalous dimension at critical wrapping order in $N=4$ SYM, JHEP 03 (2010) 103 [arXiv:0908.0234] [SPIRES].

[13] G. Arutyunov and S. Frolov, Thermodynamic Bethe Ansatz for the $\mathrm{AdS}_{5} \times \mathrm{S}^{5}$ Mirror Model, JHEP 05 (2009) 068 [arXiv: 0903.0141] [SPIRES].

[14] G. Arutyunov and S. Frolov, Simplified TBA equations of the $\mathrm{AdS}_{5} \times \mathrm{S}^{5}$ mirror model, JHEP 11 (2009) 019 [arXiv:0907.2647] [SPIRES].

[15] D. Bombardelli, D. Fioravanti and R. Tateo, Thermodynamic Bethe Ansatz for planar AdS/CFT: a proposal, J. Phys. A 42 (2009) 375401 [arXiv:0902.3930] [SPIRES].

[16] N. Gromov, V. Kazakov, A. Kozak and P. Vieira, Exact Spectrum of Anomalous Dimensions of Planar $N=4$ Supersymmetric Yang-Mills Theory: TBA and excited states, Lett. Math. Phys. 91 (2010) 265 [arXiv:0902 .4458] [SPIRES].

[17] G. Arutyunov, S. Frolov and R. Suzuki, Exploring the mirror TBA, JHEP 05 (2010) 031 [arXiv:0911.2224] [SPIRES].

[18] J. Ambjørn, R.A. Janik and C. Kristjansen, Wrapping interactions and a new source of corrections to the spin-chain/string duality, Nucl. Phys. B 736 (2006) 288 [hep-th/0510171] [SPIRES].

[19] G. Arutyunov and S. Frolov, On String S-matrix, Bound States and TBA, JHEP 12 (2007) 024 [arXiv:0710.1568] [SPIRES].

[20] G. Arutyunov and S. Frolov, String hypothesis for the $\mathrm{AdS}_{5} \times \mathrm{S}^{5}$ mirror, JHEP 03 (2009) 152 [arXiv:0901.1417] [SPIRES]. 
[21] G. Arutyunov, S. Frolov and R. Suzuki, Five-loop Konishi from the Mirror TBA, JHEP 04 (2010) 069 [arXiv: 1002.1711] [SPIRES].

[22] J. Balog and A. Hegedus, 5-loop Konishi from linearized TBA and the XXX magnet, JHEP 06 (2010) 080 [arXiv:1002.4142] [SPIRES].

[23] N. Gromov, V. Kazakov and P. Vieira, Exact Spectrum of Planar $\mathcal{N}=4$ Supersymmetric Yang- Mills Theory: Konishi Dimension at Any Coupling, Phys. Rev. Lett. 104 (2010) 211601 [arXiv:0906.4240] [SPIRES].

[24] S. Frolov, Konishi operator at intermediate coupling, J. Phys. A 44 (2011) 065401 [arXiv: 1006.5032] [SPIRES].

[25] J. Balog and A. Hegedus, The Bajnok-Janik formula and wrapping corrections, JHEP 09 (2010) 107 [arXiv: 1003.4303] [SPIRES].

[26] A.B. Zamolodchikov, On the thermodynamic Bethe ansatz equations for reflectionless ADE scattering theories, Phys. Lett. B 253 (1991) 391 [SPIRES].

[27] N. Gromov, V. Kazakov and P. Vieira, Exact Spectrum of Anomalous Dimensions of Planar $N=4$ Supersymmetric Yang-Mills Theory, Phys. Rev. Lett. 103 (2009) 131601 [arXiv:0901.3753] [SPIRES].

[28] A. Hegedus, Discrete Hirota dynamics for AdS/CFT, Nucl. Phys. B 825 (2010) 341 [arXiv: 0906.2546] [SPIRES].

[29] S. Frolov and R. Suzuki, Temperature quantization from the TBA equations, Phys. Lett. B 679 (2009) 60 [arXiv:0906. 0499] [SPIRES].

[30] A. Cavaglia, D. Fioravanti and R. Tateo, Extended Y-system for the $A d S_{5} / C F T_{4}$ correspondence, Nucl. Phys. B 843 (2011) 302 [arXiv: 1005.3016] [SPIRES].

[31] A.B. Zamolodchikov and A.B. Zamolodchikov, Factorized S-matrices in two dimensions as the exact solutions of certain relativistic quantum field models, Annals Phys. 120 (1979) 253 [SPIRES].

[32] G. Arutyunov, S. Frolov and M. Staudacher, Bethe ansatz for quantum strings, JHEP 10 (2004) 016 [hep-th/0406256] [SPIRES].

[33] M. Staudacher, The factorized S-matrix of CFT/AdS, JHEP 05 (2005) 054 [hep-th/0412188] [SPIRES].

[34] N. Beisert, The SU(2|2) dynamic S-matrix, Adv. Theor. Math. Phys. 12 (2008) 945 [hep-th/0511082] [SPIRES].

[35] R. Hernandez and E. Lopez, Quantum corrections to the string Bethe ansatz, JHEP 07 (2006) 004 [hep-th/0603204] [SPIRES].

[36] N. Beisert, R. Hernandez and E. Lopez, A crossing-symmetric phase for $A d S_{5} \times S^{5}$ strings, JHEP 11 (2006) 070 [hep-th/0609044] [SPIRES].

[37] N. Beisert, B. Eden and M. Staudacher, Transcendentality and crossing, J. Stat. Mech. (2007) P01021 [hep-th/0610251] [SPIRES].

[38] D.V. Bykov and S. Frolov, Giant magnons in TsT-transformed $\mathrm{AdS}_{5} \times \mathrm{S}^{5}$, JHEP 07 (2008) 071 [arXiv: 0805.1070] [SPIRES].

[39] E.K. Sklyanin, Boundary Conditions for Integrable Quantum Systems, J. Phys. A 21 (1988) 2375 [SPIRES]. 
[40] P. Dorey and R. Tateo, Excited states by analytic continuation of TBA equations, Nucl. Phys. B 482 (1996) 639 [hep-th/9607167] [SPIRES].

[41] J. Balog and A. Hegedus, TBA equations for excited states in the sine-Gordon model, J. Phys. A 37 (2004) 1903 [hep-th/0304260] [SPIRES].

[42] J. Teschner, On the spectrum of the Sinh-Gordon model in finite volume, Nucl. Phys. B 799 (2008) 403 [hep-th/0702214] [SPIRES].

[43] S.A. Frolov, R. Roiban and A.A. Tseytlin, Gauge-string duality for superconformal deformations of $N=4$ super Yang-Mills theory, JHEP 07 (2005) 045 [hep-th/0503192] [SPIRES].

[44] F. Fiamberti, A. Santambrogio, C. Sieg and D. Zanon, Single impurity operators at critical wrapping order in the beta-deformed $N=4$ SYM, JHEP 08 (2009) 034 [arXiv:0811.4594] [SPIRES].

[45] F. Fiamberti, A. Santambrogio, C. Sieg and D. Zanon, Finite-size effects in the superconformal beta-deformed $N=4$ SYM, JHEP 08 (2008) 057 [arXiv:0806.2103] [SPIRES].

[46] N. Gromov and F. Levkovich-Maslyuk, $Y$-system and beta-deformed $N=4$ super-Yang-Mills, J. Phys. A 44 (2011) 015402 [arXiv: 1006.5438] [SPIRES].

[47] C. Ahn, Z. Bajnok, D. Bombardelli and R.I. Nepomechie, Finite-size effect for four-loop Konishi of the beta-deformed $N=4$ SYM, Phys. Lett. B 693 (2010) 380 [arXiv: 1006.2209] [SPIRES].

[48] J. Gunnesson, Wrapping in maximally supersymmetric and marginally deformed $N=4$ Yang-Mills, JHEP 04 (2009) 130 [arXiv:0902.1427] [SPIRES].

[49] L.F. Alday, G. Arutyunov and S. Frolov, New integrable system of 2dim fermions from strings on $A d S_{5} \times S^{5}$, JHEP 01 (2006) 078 [hep-th/0508140] [SPIRES].

[50] G. Arutyunov and S. Frolov, Foundations of the $\mathrm{AdS}_{5} \times \mathrm{S}^{5}$ Superstring. Part I, J. Phys. A 42 (2009) 254003 [arXiv:0901.4937] [SPIRES].

[51] G. Arutyunov, S. Frolov, J. Plefka and M. Zamaklar, The off-shell symmetry algebra of the light-cone $A d S_{5} \times S^{5}$ superstring, J. Phys. A 40 (2007) 3583 [hep-th/0609157] [SPIRES].

[52] K. Ideguchi, Semiclassical strings on $A d S / Z(M)$ and operators in orbifold field theories, JHEP 09 (2004) 008 [hep-th/0408014] [SPIRES].

[53] N. Beisert and R. Roiban, The Bethe ansatz for $Z(S)$ orbifolds of $N=4$ super Yang-Mills theory, JHEP 11 (2005) 037 [hep-th/0510209] [SPIRES].

[54] O. Lunin and J.M. Maldacena, Deforming field theories with $\mathrm{U}(1) \times \mathrm{U}(1)$ global symmetry and their gravity duals, JHEP 05 (2005) 033 [hep-th/0502086] [SPIRES].

[55] S. Frolov, Lax pair for strings in Lunin-Maldacena background, JHEP 05 (2005) 069 [hep-th/0503201] [SPIRES].

[56] N. Beisert and R. Roiban, Beauty and the twist: The Bethe ansatz for twisted $N=4 S Y M$, JHEP 08 (2005) 039 [hep-th/0505187] [SPIRES].

[57] S.A. Frolov, R. Roiban and A.A. Tseytlin, Gauge-string duality for (non)supersymmetric deformations of $N=4$ super Yang-Mills theory, Nucl. Phys. B 731 (2005) 1 [hep-th/0507021] [SPIRES]. 
[58] L.F. Alday, G. Arutyunov and S. Frolov, Green-Schwarz strings in TsT-transformed backgrounds, JHEP 06 (2006) 018 [hep-th/0512253] [SPIRES].

[59] G. Arutyunov, S. Frolov and M. Zamaklar, The Zamolodchikov-Faddeev algebra for $A d S_{5} \times S^{5}$ superstring, JHEP 04 (2007) 002 [hep-th/0612229] [SPIRES].

[60] V.V. Bazhanov, T. Lukowski, C. Meneghelli and M. Staudacher, A Shortcut to the Q-Operator, J. Stat. Mech. (2010) P11002 [arXiv: 1005.3261] [SPIRES].

[61] G. Arutyunov, M. de Leeuw, R. Suzuki and A. Torrielli, Bound State Transfer Matrix for $\mathrm{AdS}_{5} \times \mathrm{S}^{5}$ Superstring, JHEP 10 (2009) 025 [arXiv:0906.4783] [SPIRES].

[62] G. Arutyunov, M. de Leeuw and A. Torrielli, On Yangian and Long Representations of the Centrally Extended SU(2|2) Superalgebra, JHEP 06 (2010) 033 [arXiv:0912.0209] [SPIRES].

[63] V. Bazhanov and N. Reshetikhin, Restricted solid on solid models connected with simply based algebras and conformal field theory, J. Phys. A 23 (1990) 1477 [SPIRES].

[64] A. Kuniba, T. Nakanishi and J. Suzuki, Functional relations in solvable lattice models. 1: Functional relations and representation theory, Int. J. Mod. Phys. A 9 (1994) 5215 [hep-th/9309137] [SPIRES].

[65] Z. Tsuboi, A note on the osp(1-2s) thermodynamic Bethe ansatz equation, Int. J. Mod. Phys. A 17 (2002) 2351 [cond-mat/0108358] [SPIRES].

[66] C. Gomez, J. Gunnesson and R. Hernandez, Magnons and BFKL, JHEP 09 (2008) 060 [arXiv:0807.2339] [SPIRES].

[67] M. Beccaria and G.F. De Angelis, On the wrapping correction to single magnon energy in twisted $N=4$ SYM, Int. J. Mod. Phys. A 24 (2009) 5803 [arXiv:0903.0778] [SPIRES].

[68] G. Arutyunov and S. Frolov, The Dressing Factor and Crossing Equations, J. Phys. A 42 (2009) 425401 [arXiv: 0904.4575] [SPIRES].

[69] A.V. Kotikov and V.N. Velizhanin, Analytic continuation of the Mellin moments of deep inelastic structure functions, hep-ph/0501274 [SPIRES]. 